\title{
Social and Physical Cognition in Old World Monkeys A Comparative Perspective
}

\author{
Dissertation \\ for the award of the degree \\ "Doctor rerum naturalium" \\ Division of Mathematics and Natural Sciences \\ of the Georg-August-Universität Göttingen
}

submitted by

Vanessa Schmitt

from Trier

Göttingen 2012 
Cognitive Ethology Lab

German Primate Center (DPZ)

Kellnerweg 4

37077 Göttingen

Prof. Dr. Michael Waldmann (Second Referee)

Department of Psychology

Georg-Elias-Müller-Institute of Psychology

University of Göttingen

Gosslerstr. 14

37073 Göttingen

Prof. Dr. Hannes Rakoczy

Department of Developmental Psychology

Georg-Elias-Müller Institute of Psychology

University of Göttingen

Waldweg 26

37073 Göttingen

Prof. Dr. Andreas Nieder

Department of Animal Physiology

University of Tübingen

Auf der Morgenstelle 28

72076 Tübingen

External thesis advisors:

Prof. Dr. Julia Ostner

Courant Research Center Evolution of Social Behavior Research Group Social Evolution in Primates

Kellnerweg 6

37077 Göttingen

Prof. Dr. Peter Kappeler

Behavioral Ecology \& Sociobiology Unit

German Primate Center (DPZ)

Kellnerweg 4

37077 Göttingen

Date of the oral examination: 13. April, 2012 
Herewith I declare that I have written this thesis independently and with no other aids and sources than quoted.

Göttingen, 08.03.2012

Vanessa Schmitt 



\section{Table of Contents}

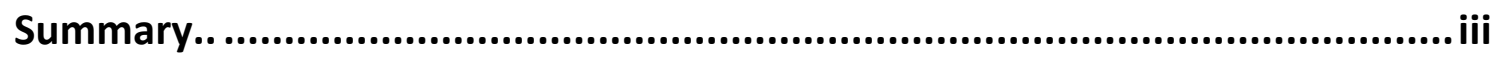

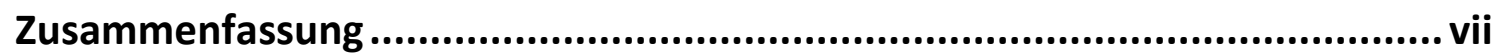

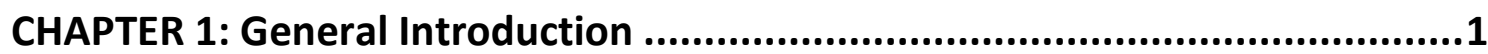

1.1. The Advent of Comparative Psychology .................................................................... 2

1.2. Theories on (Primate) Cognitive Evolution..................................................................... 4

1.3. Anthropomorphism in Comparative Psychology ......................................................... 8

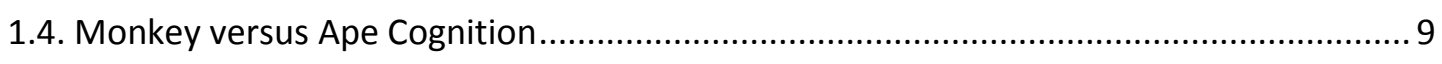

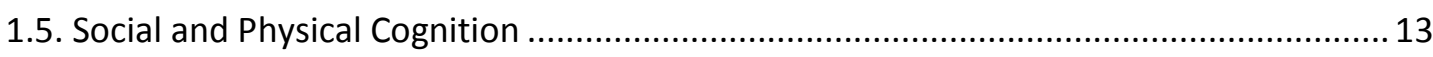

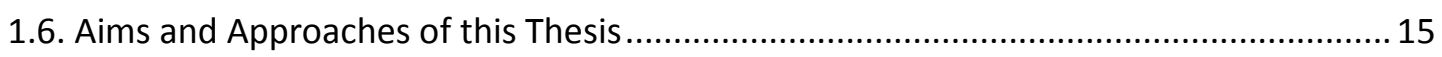

CHAPTER 2: Old World Monkeys Compare to Apes in the

Primate Cognition Test Battery.................................................17

CHAPTER 3: Representational Format Determines Numerical

Competence in Monkeys ...........................................................49

CHAPTER 4: Food, Sex, and the Brain: Which Factors Influence

Size Discrimination in Primates...................................................61

CHAPTER 5: Now you see me, now you don't - Social Inhibition

of the Use of Pointing Cues...........................................................77

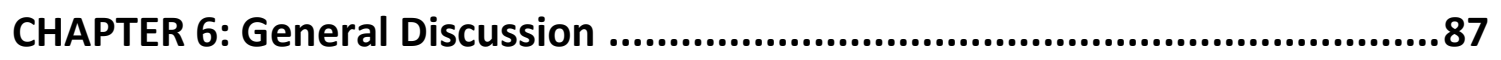

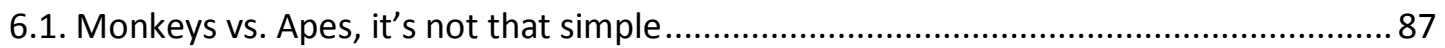

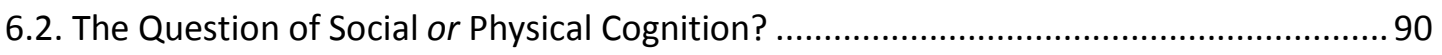

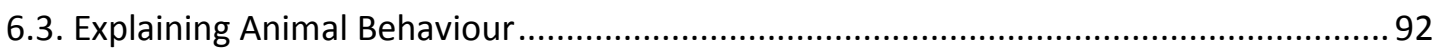

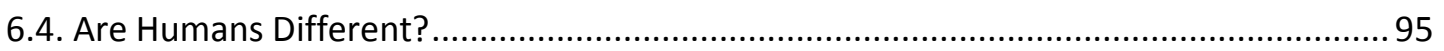

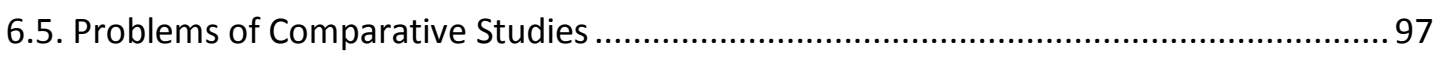

6.6. Suggestions for Future Studies.................................................................................. 99

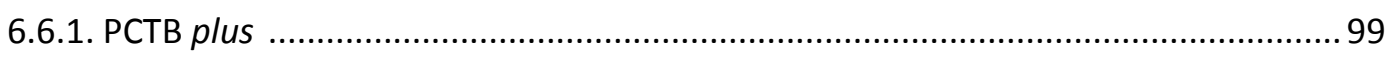




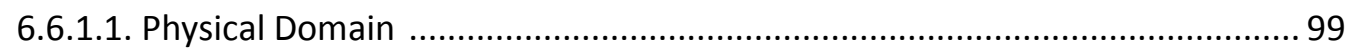

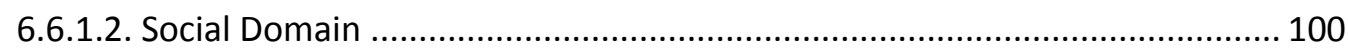

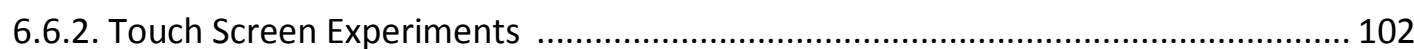

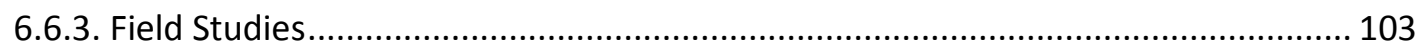

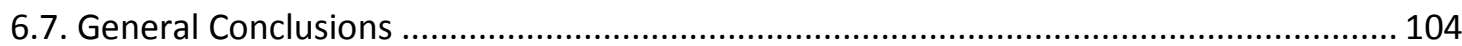

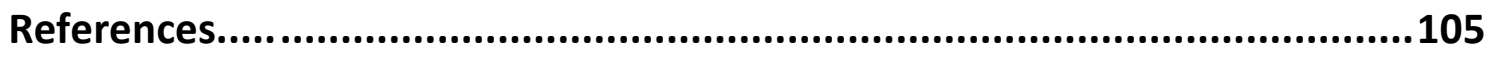

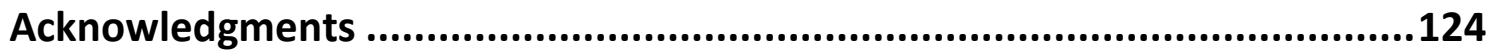

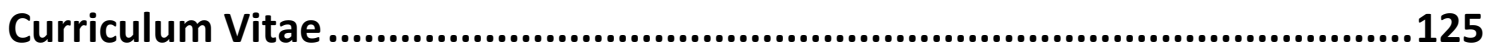




\section{Summary}

Primates have brains that are approximately twice as large as those of other similarly-sized mammals. Furthermore, also within the primate order brains have not only become increasingly large, but have also revealed a significant increase in cortical mass. The social brain hypothesis posits that this increase is due to the demands of life in a complex social group, and data regarding the relationship between brain size and social complexity support this view. Whether this pressure only affects 'social intelligence' or leads to higher general cognitive capacities (including physical cognition) remains unclear. Furthermore, whether an increase in brains size predicts an increase in intelligence is highly debated. In the past years the bulk of research on the evolution of primate intelligence focused primarily on great apes and human children. To develop a full understanding of the evolutionary dynamics of primate intelligence, however, comparative data for monkeys are needed.

The first aim of my $\mathrm{PhD}$ thesis was to provide a systematic interspecific comparison among and between apes and monkeys, covering a wide range of cognitive tasks. I tested two Old World monkey species (olive baboons and long-tailed macaques) in the so-called Primate Cognition Test Battery (PCTB), which was developed by Herrmann and colleagues (2007) to test great apes and human children. Surprisingly, our tests revealed largely comparable results between Old World monkeys and great apes. Single comparisons showed that chimpanzees performed better than the baboons and macaques in experiments on spatial understanding and tool use only, but in none of the socio-cognitive tasks. These results question the clear-cut relationship between cognitive performance and brain size. However, as the experiments of this test battery were devised to tap into human-specific skills in the first place, the experiments may underestimate true nonhuman primate competencies and species differences.

To get a better understanding of the underlying cognitive mechanisms that determined the monkeys' performances and to assess the possible influence of different socio-ecological and phylogenetic factors on their capabilities, I analysed selected tasks in more detail and extended the test paradigms. In terms of physical cognition I examined the monkeys' quantity and size discrimination abilities, while in terms of social cognition I analysed their understanding of human pointing cues in more detail.

In the PCTB experiments on quantity discrimination we used different amounts of food items to test the monkeys, which yielded relatively poor results. The further analyses of their numerical abilities revealed that quantity discrimination was indeed significantly enhanced when the subjects were tested with inedible items compared to food items. More importantly, when the monkeys were tested with food, but rewarded with other food items, the accuracy was equally high. These results indicate that not the physical quality of the stimuli determined their performance, but how they represented them, i.e. as choice stimuli or food. The replacement of the reward apparently facilitated the representation of the food items as signifiers for other foods, which in turn supported a higher acuity in decision making. This study demonstrated that quantity discrimination paradigms using food may underestimate the true 
competency of a species and provided further insight into the conditions that favour rational decision making, namely decreasing the appetitive value of a choice stimulus. Similar results have been obtained in studies with human children suggesting that the basic cognitive operations that facilitate abstract reasoning have deep evolutionary roots.

Adding a physico-cognitive experiment not included in the PCTB, furthermore allowed us to assess whether environmental challenges may have differently influenced the evolution of perceptual and cognitive capacities in nonhuman primate species. We tested the abilities for fine-grained size-discrimination in three great ape species (done by Iris Körger at the Wolfgang Köhler Primate Research Center in Leipzig) and the olive baboons and long-tailed macaques at the German Primate Center. We found that species with a frugivorous diet (chimpanzees, bonobos, baboons, macaques) were able to discriminate three-dimensional cubes with a difference in size of only $10 \%$ (i.e. $2 \mathrm{~mm}$ side length). Moreover, the successful species were even able to discriminate the cubes after successive presentations with a time lag of $60 \mathrm{~s}$. Gorillas, a predominantly folivorous species, did not discriminate between objects with $30 \%$ size difference (i.e. $6 \mathrm{~mm}$ ), but managed to distinguish between objects differing $60 \%$ in size. In contrast to diet, the presence of exaggerated sexual swellings and brain size seemed not to account for variation in performance.

Finally, to further examine the monkeys' socio-cognitive abilities, I tested whether the presence of the experimenter inhibits them to use a human pointing cue. According to the cultural intelligence hypothesis humans evolved special skills for cooperative communication. Supporting this assumption, nonhuman primates typically fail to use human communicative cues like pointing to find rewards (which they also did in the PCTB) and do not even easily learn to use these cues. We now found that the monkeys successfully used pointing cues in an objectchoice paradigm when only the arm of the experimenter was visible, while the rest of the body was hidden behind a curtain. The monkeys also used the cue, when I pointed with a doll's arm or a stick to the baited cup and their performance was significantly better when the distance between stimulus and target was close. Intriguingly, after these experiments, the monkeys' performance was also significantly improved with the experimenter being visible. Apparently, the monkeys were first distracted by the perception of the experimenter, but then learned to use the cue. The results suggest that for the use of a human pointing gesture a special understanding of its communicative intent is not necessary. Instead, local enhancement may account for much of the monkeys' performance, questioning some of the assumptions about species-specific differences in the ability to comprehend communicative gestures.

In conclusion, the experiments described in this dissertation suggest that monkeys possess similar cognitive abilities as apes, at least in the experiments conducted here. In the tests of the PCTB the apes' performance was more similar to monkeys than to human children, which excelled primarily in the social tasks of the PCTB, supporting the view of an accelerated evolution of social intelligence in humans. Our experiments revealed that monkeys understand physical relations and are able to use the behaviour of others, for example to find a food 
reward. However, they do not seem to understand the intentional structure of communicative actions as shown in the pointing study. My analyses showed that a further examination of specific experiments can reveal significant influences of methodological aspects and provided a better understanding of the underlying cognitive mechanisms. Finally, species' ecologies seem to account for much more variation than phylogenetic relatedness or brain size and should be considered in future studies. 


\section{Zusammenfassung}

Primaten haben im Vergleich zu anderen Säugetieren mit vergleichbarer Größe doppelt so große Gehirne, wobei sich auch innerhalb des Taxons der Primaten ein signifikanter Trend zur Vergrößerung insbesondere des Kortex beobachten lässt. Relativ zur Körpergröße besitzen Menschenaffen tendenziell größere Gehirne als Tieraffen, deren Gehirne wiederum größer sind als die der Halbaffen. Die sogenannte Social Brain Hypothese besagt, dass die Vergrößerung des Gehirns durch den selektiven Druck des Lebens in komplexen sozialen Gesellschaften entstanden ist. Unklar bleibt, ob dieser Druck lediglich zu erhöhten sozialen kognitiven Fähigkeiten oder allgemein zu höheren kognitiven Leistungen führt (also auch die physikalische Kognition umfasst) und ob ein größeres Gehirn tatsächlich mit gesteigerten kognitiven Fähigkeiten gleichgesetzt werden kann.

Erstes Ziel dieser Doktorarbeit war es die physiko- und sozio-kognitiven Fähigkeiten von Tieraffen einem systematischen Vergleich mit Menschenaffen zu unterwerfen, um die evolutionären Ursprünge der genannten Fähigkeiten besser phylogenetisch einordnen zu können. Dazu testeten wir die sozialen und physikalischen Kompetenzen von Anubispavianen und Javaneraffen (Makaken) am Deutschen Primatenzentrum (DPZ) mit Hilfe der Primate Cognition Test Battery (PCTB), die von Herrmann und Kollegen (2007) zum Test von Menschenaffen und Kleinkindern entwickelt wurde. Die Experimente der PCTB lieferten überraschend ähnliche Resultate für Tier- und Menschenaffen. Einzelvergleiche zeigten, dass Schimpansen nur in Versuchen zum räumlichen Verständnis und Werkzeuggebrauch etwas besser abschnitten als die Paviane und Makaken. Hingegen fanden wir keine Steigerung soziokognitiver Fähigkeiten von Tier- zu Menschenaffen. Diese Ergebnisse stellen die Hypothese, dass ein größeres Gehirn mit einer Steigerung der Intelligenz einhergeht, in Frage. Allerdings wurden die Experimente anfänglich entwickelt um spezifisch menschliche Fähigkeiten zu untersuchen, daher könnten sowohl die Fähigkeiten der Affen als auch Artunterschiede unterschätzt worden sein.

Um die zugrundeliegenden kognitiven Mechanismen näher zu analysieren und den möglichen Einfluss ökologischer Faktoren auf die Entwicklung bestimmter Fähigkeiten zu berücksichtigen, erweiterte ich daher spezifische Testparadigmen der РСTB und führte zusätzliche Versuche durch. Im Hinblick auf die physikalische Kognition testeten wir inwieweit die Tiere Mengen und Größen unterscheiden können; im Bereich der sozialen Kognition analysierte ich ihr Verständnis der menschlichen Zeigegeste.

In den Versuchen der РСТВ wählten die Paviane und Makaken die größere von zwei Futtermengen in nur circa 70\% der Fälle. Die weitergehende Untersuchung ihrer numerischen Fähigkeiten zeigte, dass sie bedeutend besser zwischen verschiedenen Mengen unterscheiden konnten, wenn es sich hierbei um nicht-essbare Stimuli handelte (85\% korrekt). Interessanterweise erreichten die Affen die gleiche Leistung, wenn die zu diskriminierenden Stimuli zwar aus Futter (Rosinen) bestanden, sie aber als Belohnung anderes Futter bekamen. Die Ergebnisse deuten darauf hin, dass nicht die physikalische Beschaffenheit der Stimuli (essbar 
oder nicht) die Leistung der Affen beeinflusste, sondern dass wofür sie diese hielten (Belohnung oder Reizstimulus). Die Tiere waren durch den Austausch der Belohnung offensichtlich in der Lage Rosinen als Anzeiger für andere Rosinen wahrzunehmen, was wiederum zu einer gesteigerten Entschlussfähigkeit führte. Diese Versuche zeigen, dass numerische Studien mit Futterstimuli wahrscheinlich die Fähigkeiten von Tieren unterschätzen, und dass die Senkung des appetitiven Wertes eines Stimulus die Entscheidungsfindung verbessern kann. Diese Resultate gleichen denen in Kinderstudien und deuten darauf hin, dass sich die grundlegenden kognitiven Mechanismen abstrakten Denkens evolutionär bereits früh ausgebildet haben.

Die Versuche zur Größenunterscheidung bei Primaten lieferten interessante Hinweise darauf, welche ökologischen Faktoren diese Fähigkeit beeinflusst haben könnten. Insgesamt testeten wir drei Menschenaffenarten (durchgeführt von Iris Kröger am Wolfgang-KöhlerPrimaten-Forschungszentrum in Leipzig), sowie die Anubispaviane und Javaneraffen am DPZ. Die Arten, die sich vorwiegend von Früchten ernähren, waren in der Lage minimale Größenunterschiede von Quadern zu erkennen (10\%, i.e. $2 \mathrm{~mm}$ Seitenlänge) und wählten den größeren Quader auch nach einer schrittweisen Präsentation mit bis zu 60 Sekunden Zeitverzögerung. Gorillas, die sich hauptsächlich von Blättern ernähren, lernten im Gegensatz dazu nur zwischen relativ großen Volumenunterschieden zu diskriminieren (60\%). Unterschiedlich ausgeprägte Sexualschwellungen sowie die Hirngröße hatten hingegen keinen signifikanten Einfluss auf die Leistung der Tiere. Diese Ergebnisse verdeutlichen, wie wichtig die Berücksichtigung ökologischer Faktoren in vergleichenden Experimenten sein kann, die die Evolution kognitiver und perzeptueller Fähigkeiten beeinflusst haben könnten.

Im letzten Experiment habe ich schließlich untersucht, ob die Anwesenheit des Experimentators die Affen daran hindert eine Zeigegeste zu nutzen. Der „kulturellen Intelligenzhypothese“ zufolge entwickelten nur Menschen spezielle Fähigkeiten zur kooperativen Kommunikation. Versuche mit nicht-menschlichen Primaten unterstützen diese Annahme, da diese typischerweise nicht in der Lage sind menschliche Kommunikationssignale zu nutzen um Futter zu finden und zudem Schwierigkeiten haben dies zu lernen. In unseren Versuchen zeigte sich allerdings, dass Affen durchaus die Zeigegeste nutzen können, wenn nur der Arm des Experimentators sichtbar ist. Die Affen wählten den befüllten Becher auch, wenn ich einen Puppenarm oder einen Stock benutzte um darauf zu zeigen. Die Tiere waren allerdings nur erfolgreich, wenn die Entfernung zwischen Stimulus und Becher gering war. Interessanterweise, konnten die Tiere nach diesen Versuchen den Zeigehinweis auch nutzen, wenn ich wieder vor innen stand. Offensichtlich waren sie anfänglich durch die Anwesenheit des Menschen abgelenkt, lernten dann aber die Geste zu nutzen. Diese Ergebnisse deuten darauf hin, dass es nicht nötig ist die zugrundeliegende kommunikative Intention dieses Signals zu verstehen. Im Gegenteil, ein Großteil der Performanz kann wahrscheinlich durch lokale Verstärkung erklärt werden, was die Annahmen über bedeutende Artunterschiede im Verständnis kommunikativer Gesten in Frage stellt. 
Die Ergebnisse dieser Dissertation weisen darauf hin, dass Tieraffen in den hier untersuchten Bereichen nicht weniger intelligent sind als Menschenaffen. Im Gegenteil, die Performanz der Menschenaffen war in den Experimenten der PCTB der der Paviane und Makaken ähnlicher als der der Kinder, welche die Affen hauptsächlich in den sozio-kognitiven Experimenten übertrafen. Soziale Faktoren scheinen also vorrangig in der Entwicklung des Menschen von Bedeutung gewesen zu sein. Die Ergebnisse zeigen weiterhin, dass Tieraffen durchaus in der Lage sind physikalische Zusammenhänge zu verstehen und das Verhalten von anderen nutzen können, um zum Beispiel Futter zu finden. Allerdings scheint ihnen ein grundlegendes Verständnis der intentionalen Struktur kommunikativer Prozesse zu fehlen. Des Weiteren veranschaulichten die durchgeführten Experimente, dass ökologische Faktoren Artunterschiede wahrscheinlich besser erklären können als Hirngröße oder phylogenetische Verwandtschaft. Die systematische Analyse spezifischer Paradigmen zeigte letztlich, dass methodische Aspekte einen entscheidenden Einfluss auf die Ergebnisse haben können, und führte zu einem besseren Verständnis der zugrundeliegenden kognitiven Mechanismen. 


\section{General Introduction}

How do cognitive abilities evolve? Since Darwin (1871), this question preoccupied the minds of many researchers in various disciplines (Dennett 1987; Richards 1987; Byrne \& Whiten 1988; Matsuzawa 2001; Gazzaniga et al. 2002; Russon \& Begun 2004; Pinker 2010) and is continuously debated (e.g. Barrett 2011; Menzel \& Fischer 2011; Nunn 2011; Reader et al. 2011; De Waal \& Ferrari 2012). Cognition is typically defined as the mental processes by which we acquire, process, store and act on information from the environment such as learning, memory and decision-making (Shettleworth 2010b). Owing to our human nature, past research has focused mainly on identifying our (seemingly) unique cognitive capacities (Kappeler \& Silk 2010; Penn 2011). As great apes are our closest living relatives, the last decades were dominated by human - ape (in particular chimpanzee) comparisons to elucidate "what makes us human" (see Herrmann et al. 2007). However, as Rogers and Kaplan (2004) stated "[...] we are beginning to become aware of the risk of moving in one intellectual milieu and choosing to work on a species instead of choosing a problem and then testing it on a range of species" (p. vi). Studies using comparative methods have been flourishing in the last couple of years, as more and more researchers begin to acknowledge the importance of comparing different species to understand cognition (MacLean et al. 2011). Since the end of the $20^{\text {th }}$ century, primate cognition in particular has been receiving much renewed interest and researchers now include additional species into the picture (Banerjee et al. 2009; Amici et al. 2010; Yocom 2010; Sandel et al. 2011; Burkart \& van Schaik 2012)

However, one difficulty is that in the last decades a large part of ape cognition studies has been conducted in the framework of developmental psychology using experimental setups in a laboratory (e.g. Tomasello \& Call 1997; Matsuzawa 2003), whereas monkeys were primarily studied in the field, using an ecological approach to explain their behaviour (see Cheney \& Seyfarth 1992, 2008; but see Hauser 1997; or Hauser et al. 1999 for pioneering experimental studies with monkeys). Data comparing monkeys and apes in a controlled experimental setup are therefore still scarce (for example Amici et al. 2010). To contribute to the understanding of primate cognition one focus of the present project was to compare the cognitive capacities of two Old World monkey species, olive baboons (Papio anubis) and long-tailed macaques (Macaca fascicularis), to those of the great apes in a large set of experiments (i.a. the so-called Primate Cognition Test Battery [PCTB], Herrmann 2007). In addition, by systematically examining factors influencing subject's performances in the experiments (for example the quality of the experimental stimuli) I further tried to elucidate the underlying cognitive mechanisms. 
In the following ( will 1) briefly review the history of comparative psychology 2) describe the present theories on cognitive evolution, 3) consider the problems of an anthropomorphic approach on cognition, 4) comment on the suggested monkey-ape dichotomy and 5) outline which aspects of social and physical cognition I examine in this thesis.

\subsection{The Advent of Comparative Psychology}

Since the rise of comparative psychology, one major aim has been to elucidate the processes of cognitive evolution. Darwin's famous statement that "the difference in mind between man and the higher animals [...] certainly is one of degree and not of kind" (Darwin, 1871 , p. 105) stimulated not only the search for human-like behaviours in animals, but at the same time the search for our unique cognitive abilities (Kappeler \& Silk 2010). The mental continuity proposed by Darwin would suppose that the cognition of Homo sapiens should share many features with closely related species. Similarities due to phylogenetic relatedness are called homologies (Geissmann 2003). But similar cognitive abilities could also be due to convergent evolution of species facing the same ecological or social challenges (called analogies). Examining the similarities and differences between closely related species is a prerequisite to identify the dynamics in the evolution of cognition (Byrne 1995; Suddendorf \& Whiten 2001; Shettleworth 2010b; MacLean et al. 2011; Nunn 2011).

The main objective for many cognitive psychologists thus was and still is to understand the evolution of our own cognitive abilities (Yerkes 1943, 1971; Byrne \& Whiten 1988; Dunbar 1992; Povinelli 1993; Hauser 1996; Matsuzawa 2001; Barrett et al. 2002; Hare 2007; Herrmann et al. 2007; Premack 2007; Haun et al. 2010; Kappeler \& Silk 2010; Tomasello \& Herrmann 2010). Besides the study of ontogeny of cognitive abilities in human children (see for example Rakoczy \& Tomasello 2007; Rakoczy 2009, 2010), one major stream of research focuses on comparing the abilities of humans to their closest relatives to reconstruct the evolutionary history of human cognition. Unfortunately, all our direct ancestors are extinct and a comparison relying on fossils is insufficient as cognition does not fossilize (Lewontin 1998). Since humans and great apes (i.e. chimpanzees, bonobos, gorillas, orangutans) belong to the same phylogenetic group of hominidae and share a common ancestor that lived approximately 13-18 million years ago (Perelman et al. 2011), comparisons between these species were thought to provide the best insight into human cognitive evolution. Cognitive research has focused on apes since the beginning of the $20^{\text {th }}$ century. Köhler's (1925) and Yerkes' (1929) famous experiments with chimpanzees laid the foundation for a productive research program exploring the cognitive abilities of our closest living relatives. 
However, the studies of animal cognition were dominated by American behaviourists until the middle of the last century. Behaviourists were convinced that all animal behaviour is due to trial and error learning (Watson 1925; Skinner 1938; Harlow 1958). Thorndike (1898) suggested that actions performed by animals lack all forms of insight and that problem-solving consisted simply of associating several "more or less random and aimless [elements]" (Birch 1945, p. 367). Thus, behaviourism denied that animals possess any kind of thought or have mental experiences. Although some studies already took a comparative approach, they mainly tried to describe general cognitive processes, such as learning abilities (Thorndike 1911; Harlow et al. 1950; Harlow 1953; Bitterman 1960), not taking species ecologies or living conditions into account and often interpreting species differences within the framework of a scala naturae (i.e. an evolutionary ladder with the 'most evolved' humans ranking on top). After Thorndikes' (1911) famous experiments on various animal species, people became convinced that the general learning principles were the same for all species, leading to a decline in diversity of animal species tested and the rise of the 'lab rat'. In the 1940s Schneirla stated: "we do not have a comparative psychology [Harriman, 1946, p. 314]" (cited in Bitterman 1965, p. 396). In the 1960s Bitterman tried to improve the situation by testing a number of species, ranging from cockroaches and goldfish to turtles, rats and monkeys, finding interesting similarities and differences between species in standard laboratory tasks on learning (Bitterman 1965). Although this was a new start for comparative analyses of cognition, animals were still mostly denied any form of intelligence.

Thanks to the 'cognitive revolution' beginning in the 1960s (Griffin 1981, 1984), the radical view of behaviourism is rejected by most researchers today (Menzel \& Fischer 2011). Instead, this new approach focused on cognition as information processing and allowed the consideration of intelligent behaviour by animals. Also most experimental psychologists nowadays adopt the information-processing approach to explain for example causal understanding (Blaisdell et al. 2006; see also Penn 2011 for a review). Nonetheless, studies on the comparative cognition of primates based on a large range of species tested in a wide set of tasks were still relatively rare in the following years (see MacLean et al. 2011; Nunn 2011; Seed et al. 2011 for discussions). Call and Mendes (unpublished manuscript) revealed that, from 1978 until 2006, approximately $87 \%$ of empirical studies on ape cognition published in the Journal of Comparative Psychology were based on a single species and $68 \%$ of these were conducted with chimpanzees. This "chimpocentrism" has been criticized by various researchers (Beck 1982; Parker et al. 1999; Miklósi 2002; Sayers \& Lovejoy 2008) and highlights the problem of recent 
primate research. To develop a full understanding of the evolution of primate intelligence comparative data from monkeys and other species are needed.

There are nowadays already a number of interesting research directions focusing on nonprimate species. The second most studied animal species (the first being rats) in this respect are certainly birds, especially the family of corvids (i.a. ravens, crows, rooks, jackdaws, and nutcrackers) (Clayton \& Emery 2005) and parrots (for example the famous Alex trained and studied by Irene Pepperberg). In the last decade insightful experiments have been conducted, which indicate that these bird species possess extraordinary cognitive capacities. From tool use to deception, these birds seem to excel in nearly every cognitive domain (Emery \& Clayton 2001; Bugnyar \& Kotrschal 2004; Emery \& Clayton 2004b; Pepperberg 2004; Emery \& Clayton 2005; Pepperberg \& Gordon 2005; Pepperberg 2006; Raby et al. 2007; Bird \& Emery 2009). And not only do they outcompete other bird species, in some experiments they also perform considerably better than many primate species (Emery \& Clayton 2004b; Emery \& Clayton 2004a; Emery \& Clayton 2009). Domesticated species have also been extensively studied. Comparisons of the cognitive skills of dogs and wolves have been particularly well-investigated, as some researchers hypothesize that dogs developed specific socio-cognitive skills in the course of domestication (see Hare et al. 2002). In addition horses, sheep, cats and chickens have been in the focus of a number of cognitive studies (e.g. Dwyer \& Kendrick 2008; Rugani et al. 2008; Pisa \& Agrillo 2009; Proops et al. 2009). Likewise, cetaceans have been intensively investigated, as e.g. dolphins are considered to exhibit extraordinary cognitive skills (Pack \& Herman 2004; Marino et al. 2007; Pack 2010). Nowadays, study species range from whales and fish to bees and ants and the field of animal cognition is constantly growing (Rogers \& Kaplan 2004; Shettleworth 2010b; Menzel \& Fischer 2011). Interspecific comparisons are therefore increasingly available, and provide necessary information on the evolution of cognition. In the next section I will highlight why I focused on nonhuman primates (see also Weiss \& Santos 2006) and give an overview of the existing theories on their cognitive evolution (which are also applicable to other animal species).

\subsection{Theories on (Primate) Cognitive Evolution}

Exploring the evolution of cognition by focusing on primates has two apparent causes. First, we (Homo sapiens) are members of the primate order and have extraordinarily well-developed cognitive skills (see e.g. Boyd \& Silk 2002). Within the Hominoidea, the last common ancestor of humans and their closest living relatives, the chimpanzees and bonobos, is dated to about 6 mya (Perelman et al. 2011) (see Figure 1.1). Gorillas and orangutans split from the human 
lineage approximately 8 million and 16 million years ago, respectively. Furthermore, the chimpanzee and human genome are $98.77 \%$ identical (The Chimpanzee Sequencing and Analysis Consortium 2005). This close relatedness makes it especially interesting to investigate the cognitive abilities of apes and other primates and constitutes a promising approach to reconstruct cognitive evolution.

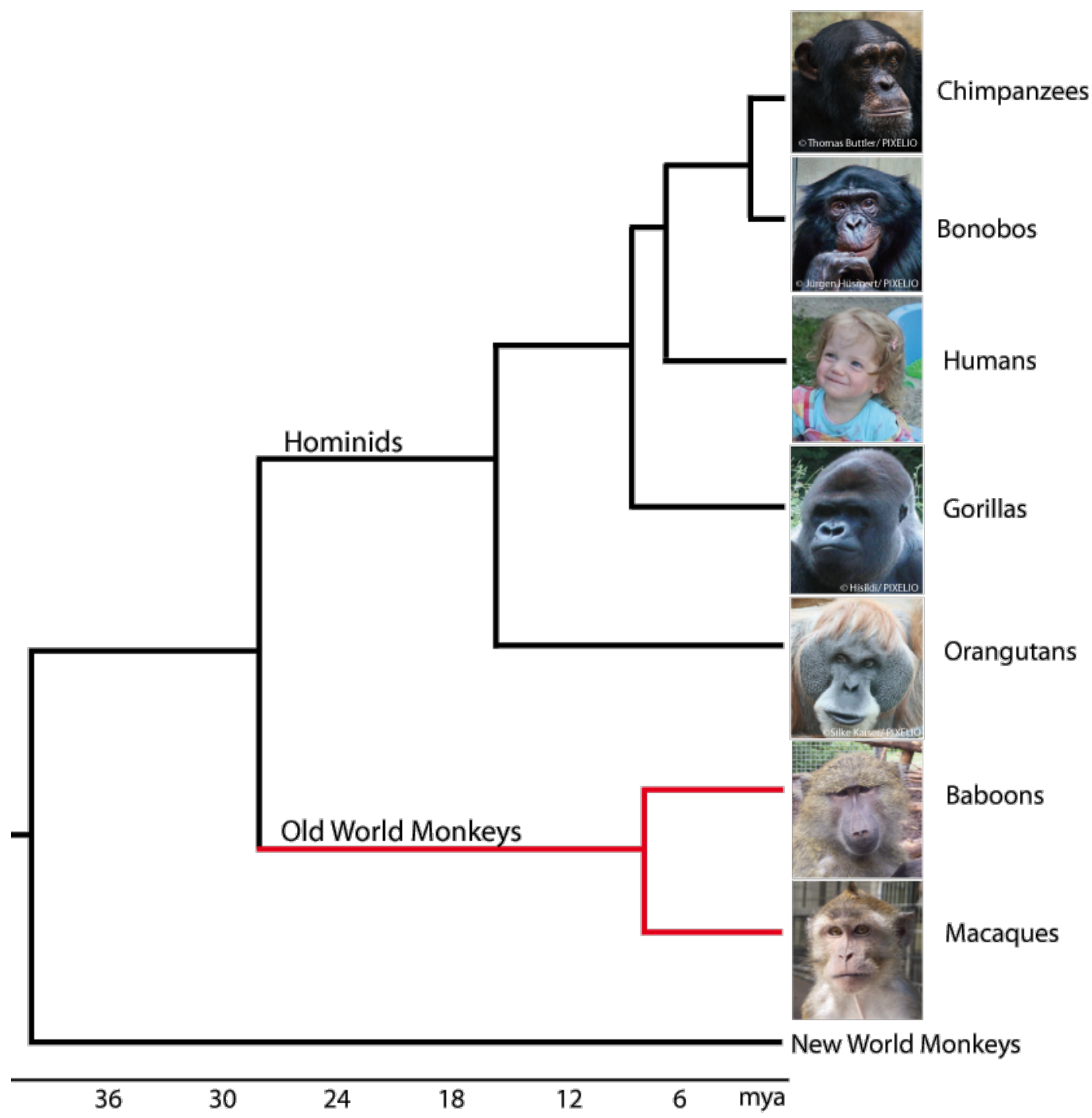

Figure 1.1: Simplified primate evolutionary tree of monkeys and apes based on genetic analysis in Perelman et al. 2011. The cladogram shows the main splits and their approximate dates of Hominids and the two Old World monkey species I focus on in this thesis (highlighted in red).

Second, compared to other mammals of comparable size, primates have brains that are approximately twice as large as expected (Passingham 1981; Barton 2006). Within the primate order, there is also a trend towards a higher relative brain size among species more closely related to humans: corrected for body size, the brains of apes tend to be larger than the brains 
of monkeys, which in turn tend to be larger than those of prosimians (Jerison 1973; Montgomery et al. 2010; Montgomery et al. 2011). Furthermore, human brains are roughly three times larger than those of great apes (Pagel \& Harvey 1989). In particular, the neocortex is the most recently evolved part of the primate brain and the area that has undergone the greatest expansion compared to other mammals. It is important for sensory perception, generation of motor commands, and higher cognition (Boyd \& Silk 2002; Carlson 2007). As a large brain is an extremely costly investment (it only accounts for $2 \%$ of our bodyweight but requires $20 \%$ of our metabolic energy, Aiello \& Wheeler 1995; Isler \& van Schaik 2006) there is still much debate about how and why these changes occurred (see also Dunbar 2009 for a review).

In the 1980s, the favoured hypothesis was that the increase in primate brain size evolved mainly in response to the especially challenging demands of foraging on patchily distributed and seasonal food (Harvey et al. 1980; Milton 1988). Another ecological hypothesis posits that species relying on hard to process food, such as hard-shelled nuts that need to be cracked open (also referred to as extractive foraging), must be more intelligent than others (Parker \& Gibson 1977; Parker \& Gibson 1979). Others proposed that the distinct aspects of primate cognition evolved mainly in response to the especially challenging demands of complex social life of constant competition and cooperation with others in the social group, termed the "Machiavellian intelligence" hypothesis by Byrne and Whiten (1988; Jolly 1966; Humphrey 1976; De Waal 1982; Dunbar 2003). In recent years, the hypothesis that primate intelligence evolved in response to the challenges of living in large and complex groups receives renewed interest (Byrne \& Bates 2010; Fitch et al. 2010; Frith \& Frith 2010), referred to as the "social-brain" or "social intelligence" hypothesis. Supporting this assertion, Dunbar and colleagues (Dunbar 1992; 1998; 2003; Dunbar \& Shultz 2007) found a relationship between primate group size and neocortex size. Furthermore, not just group size, but particularly the complexity of the social relationships (for example grooming clique size and coalition formation) seem to correlate with brain volume (Kudo \& Dunbar 2001; Dunbar \& Shultz 2007). Thus, the task of regulating one's own grooming and coalitionary relationships, while simultaneously tracking everyone else's, is viewed as a strong social selection pressure on cognitive capacities and, therefore, brain size (Kudo \& Dunbar 2001; Seyfarth \& Cheney 2007). As this is even more demanding in fissionfusion societies recent results suggest that group dynamics also influence brain size and cognition (Barrett et al. 2003; Amici et al. 2008).

Reader and colleagues (Reader \& Laland 2002; Reader et al. 2011) proposed a slightly different account on the evolution of primate intelligence. They do not support the view of 
separated social and ecological pressures, but suggest that the tendency to innovate, learn socially and use tools were the key features leading to the development of higher cognition. This assumption thus proposes an increase in general intelligence within the primate lineage in contrast to the more domain-specific accounts of the social and ecological intelligence hypotheses.

In recent years, however, a study on human children and great apes gave rise to a new and highly discussed hypothesis on the evolution of human social intelligence. As humans are not only social but "ultra-social" (Richerson \& Boyd 1998), Herrmann and colleagues (2007) posit that humans have evolved special skills to live and participate in cultural groups, in particular attention sharing, cooperation, and the attribution of mental states, which develop early in ontogeny. This so-called 'cultural intelligence hypothesis' supports the assumption that social aspects were the driving force in the evolution of intelligence, at least in the transition from apes to humans (see also Tomasello \& Moll 2009; Tomasello \& Herrmann 2010).

However, the question of whether the increase in primate brain size has actually led to an increase in cognitive abilities remains controversial (Deacon 1990; Byrne 1996; Rogers 2004). The attempt to link brain size to intelligence is fraught with problems as size per se might not be the critical factor, but the modularity and interconnectedness of different brain areas (Chittka \& Niven 2009). Furthermore, many brain areas that control primary sensory and motor functions are not associated with "intelligence" or cognition but contribute to a large brain size nevertheless (Emery \& Clayton 2004; Barton 2007). Accordingly, linking a large brain to extraordinary cognitive skills is problematic. An additional problem is that the choice of variables entered into meta-analyses trying to reveal the relation between brain size and intelligence, and the problems associated with multiple correlations impede the development of clear conclusions (Healy \& Rowe 2007). Some researchers for example proposed that overall brain size is the best predictor of cognitive abilities in nonhuman primates (Reader \& Laland 2002; Deaner et al. 2007), while others state that particular brain areas are linked to cognitive performance. According to Shultz and Dunbar (2010) the neocortex ratio and hippocampus volume are particularly important for problem solving and executive control. Robert Barton, in contrast, argues that the cerebellum is the most important brain region in the development of intelligence (Barton 2006; 2007). At a symposium on "Animal Intelligence" in August 2011 he claimed that the increase in neocortex size is primarily linked to body size and not to intelligence. The increase is actually in the white matter of the neocortex (i.e. the axons of the neurons) and not the grey matter (i.e. the number of neurons). In contrast, the cerebellum does not correlate with body size to the same extent as the neocortex does. Furthermore, the 
cerebellum encompasses four to five times more neurons than the neocortex. He concluded that volumetric ratios of the cortex are not useful for drawing conclusions about intelligence. Moreover, in a recent study, Venditti \& Barton (unpublished) found that also the cerebellum expanded throughout the primate lineage (see also Barton 2006).

\subsection{Anthropomorphism in Comparative Psychology}

"From tool use to metacognition, from deception to death, much of comparative psychology over the last 35 years has been driven by the single-minded goal of demonstrating that nonhuman animals are capable of 'human-like' cognition." Penn 2011, p.255

Focusing on primates to elucidate the development of cognition also leads to problems. Because of the strong resemblance between apes and humans, especially in morphology, physiology and development, similar behaviours were often explained by similar cognitive mechanisms. This human- (and ape) centred view in comparative psychology led to the rise of so-called "folk psychology" (Dennett 1987). Explaining animal behaviour using humancharacteristic 'mentalistic' concepts like belief, desire, knowledge, intention, understanding, self-consciousness, and so on is the core feature of this account. In his book Dennett (1987) states that "so natural and effortless are [folk psychology's] interpretations that it is almost impossible to suppress them" (p. 8). When applying these explanations to scientific research one has to be careful however. Sara Shettleworth highlighted this emerging problem: "By focusing on human-like behaviors in other species, it encouraged explanations that were often dangerously anthropomorphic in interpreting human-like behaviors as produced by human-like thought without properly considering alternatives" (Shettleworth 2012, p. 3).

Not to consider alternatives is probably the main weakness of anthropomorphism. This approach tends to explain animal behaviour in a human-like fashion, without thoroughly examining alternative explanations. A contrasting framework was offered by Tinbergen. In his famous reviews (Tinbergen 1951, 1963) he encouraged the careful causal analysis of animals' behaviours as such and to avoid interpretations in terms of anthropomorphic processes. The focus of Tinbergen and colleagues was on the study of behaviour in its ecological and evolutionary context, giving rise to today's ethology and the ecological approach on comparative cognition. The debate about the dichotomy between an anthropomorphic and ecological approach continues today (Shettleworth 2009, 2010a; Barrett 2011; Shettleworth 2012) especially when comparing humans and other primates. This dichotomy is also reflected in the different approaches used to study ape and monkey cognition mentioned earlier. 
Criticizing the anthropomorphic approach is nowadays still regarded as supporting a behaviouristic account denying all animal intelligence by some scientists (e.g. De Waal 1999, 2005,2009 ) leading to continuous discussions among researchers. Researchers focusing on ape cognition in particular disagree on the interpretations of their findings (Povinelli \& Vonk 2003; Tomasello et al. 2003b; Penn 2011), for which I will provide some examples in the next section. In this thesis I have tried to avoid purely anthropomorphic explanations of the observed results. In contrast, I used a bottom-up approach as recently emphasized by de Waal and Ferrari in their book The Primate Mind (2012). In their introductory chapter they state: "It is the [...] type of questions [...] concerning basic capacities that should drive the study of animal cognition, not an obsession with rankings and the human-animal divide. [...] Instead of asking which species can do X, the question would become how does X actually work?" (p.1). Accordingly, I analysed specific experiments in more detail to get a better understanding of the underlying cognitive mechanisms (see Chapter 3 and 5) and tried to find ecological explanations, which may account for the subjects' performances (see Chapter 4).

\subsection{Monkey versus Ape Cognition}

"Many previous studies among nonhuman primates have focused on the similarities and differences between only human and nonhuman primates. Due to the diversity of ecologies and cognitive challenges facing nonhuman primates, such a dichotomy is too broad to elucidate primate cognitive processing from an evolutionary perspective." Matsuna \& Fujita 2009, p.128

As shown in Figure 1.1, the lineages of Old World monkeys and apes are sister taxa that shared a common ancestor between 29 and 24 million years ago (Zalmout et al. 2010). Thus, comparing the cognitive skills of apes and monkeys is a prerequisite to draw inferences on the evolutionary dynamics of primate intelligence. With the increase in brain size from monkeys to apes, one would predict that apes would outperform monkeys in cognitive tasks. Byrne and Whiten (1997) further argued that there are several "principle branch points in primate evolution at which there is some evidence of intellectual change in one descendant line" (p. 14). Considering apes and monkeys they separate (a) the haplorhine line (tarsiers, Old and New World primates), which differs from the strepsirhine clade (lemurs, lorises and galagos) in having larger brains, relatively greater investment in the neocortex, and greater social complexity; and, within the haplorhine lineage (b) the great ape (Hominids) line, which has a "different level of understanding [and] absolutely larger brains" (Byrne \& Whiten 1997, p.14) and a larger neocortex ratio (Semendeferi et al. 2002; Dunbar \& Shultz 2007).

Researchers are divided in the scope that they attribute this development to social and ecological pressures (Byrne \& Whiten 1997; see Chapter 1.2.). In the framework of the social 
intelligence hypotheses one would predict that apes would outcompete monkeys especially in socio-cognitive tasks (Barrett et al. 2003; Box \& Russon 2004; Byrne \& Corp 2004; Dunbar \& Shultz 2007). However, following the assumptions of a more domain-general account, as proposed by Reader and colleagues (2011), monkeys should perform worse than apes in both social and non-social tasks.

A number of studies have provided evidence for the hypothesis that apes exceed monkeys in terms of their cognitive capacities (e.g. Byrne 1995; Tomasello \& Call 1997; Reader \& Laland 2002; Deaner et al. 2006). For example, tactical deception seems to be more common in great apes than in monkeys (Byrne and Whiten 1988) and only great apes seem to recognize themselves in mirrors (Gallup 1982; Inoue-Nakamura 1997; Anderson \& Gallup 2011). Furthermore, Deaner and colleagues (2006) argue from their meta-analysis of published nonhuman primate cognition studies that "great apes significantly outperformed other lineages" (p. 149). On the basis of previous studies, Byrne (1997; 2000) and some other researchers (Tomasello et al. 2003a, b) concluded that the difference between monkeys and apes lies mainly in their ability to form mental representations, that is to hold in mind and operate on mental objects that have semantic properties (Pitt 2008). For instance, great apes appear to understand the intentions and desires of others (see Call et al. 2004). Instead, the cognitive abilities shown by monkeys were mainly attributed to rapid learning capacities (Byrne \& Corp 2004).

On the other hand, apes may not possess higher general intelligence than monkeys, but more domain specific advances, as Herrmann and colleagues (2007) had found for human children. Recent studies by the Tomasello lab suggest that apes and monkeys differ predominantly in their socio-cognitive skills. Only 15 years ago Tomasello and Call (1997) denied large cognitive differences between apes and monkeys based on their detailed review on primate studies. However, because of new experimental paradigms and a huge increase in studies on social cognition, they changed their assumptions and now seem convinced that apes reveal extraordinary socio-cognitive skills that are probably not present in monkeys (Tomasello et al. 2003a). They state: "New data suggest that relatively drastic revisions are needed in our theoretical accounts of what other animal species understand about the psychological states of others. Specifically, chimpanzees seem to understand some things about what others do and do not see, or have and have not seen in the immediate past, as well as some things about others' goal-directed activities." (p.153).

However, the studies responsible for these assumptions suffer from some methodological weaknesses and may also be explained by simpler assumptions than the relatively 
anthropomorphic ones used by Tomasello and colleagues. There are some significant disagreements between the two major chimpanzee research groups concerning their results in 'Theory of mind' (ToM) tests (i.e. ability to attribute mental states to others, Premack \& Woodruff 1978). It is not clear whether the results from Tomasello's research group really reveal extraordinary social skills, rather than learned behaviour and behaviour-reading capacities (for a detailed discussion see Povinelli \& Vonk 2003; Tomasello et al. 2003a, b; Povinelli \& Vonk 2004; Penn \& Povinelli 2007). For example, Hare and colleagues (Hare et al. 2000; Hare et al. 2001; Hare et al. 2003) tested whether subordinate subjects go for the food items that a more dominant individual was not able to see from his point of view. As the chimpanzees (but not the capuchin monkeys) did as predicted, the researchers concluded that chimpanzees are capable of first-level perspective taking, i.e. understanding what others can and cannot see. However, Burkart and Heschl (2007) found that the tests used to draw these conclusions about understanding visual access do not require perspective taking, but can also be solved by simpler behaviour reading.

An additional problem is that many of the studies comparing apes and monkeys based their conclusions on results of experiments or observations made in different studies using different methods. Furthermore, many studies compared highly trained apes to naive monkeys, probably overestimating the differences between monkeys and apes (Tomasello \& Call 1997). Moreover, the cognitive competencies of great apes, especially chimpanzees, have been studied extensively, whereas far fewer studies have examined the capabilities of Old and New World monkeys (see Tomasello \& Call 1997 for review). Although now more comparative studies are being conducted (Shettleworth 2009; see also Yocom 2010 for a study on gibbons), systematic interspecific comparisons are still rare and comparative data for great apes and monkeys are needed.

Field experiments for example suggest that monkeys also possess extraordinary social skills. The famous studies conducted by Robert Seyfarth, Dorothy Cheney and their co-workers revealed that baboons, living in large and complex groups, have detailed knowledge of their conspecifics' social status, their rank and the relations between different animals (see Cheney \& Seyfarth 2008 for a review). A recent study on Barbary macaques indicates that these monkeys can recognize their group mates from pictures and distinguish them from unknown conspecifics (Schell et al. 2011). Other studies on monkeys propose that they may also have an understanding of others' perceptions. Amici and colleagues (2009) tested the capacities of spider monkeys, capuchin monkeys and long-tailed macaques to withhold information from others in order to gain a food reward. All monkeys performed well with interspecific differences 
best explained by their species-specific dominance hierarchies and fission-fusion dynamics. This kind of "tactical deception" was also documented in field studies on capuchin monkeys (Wheeler 2009; Wheeler 2010). Although apes perform remarkably well in the newly designed laboratory experiments on social cognition referred to by Tomasello and colleagues (2003a), it is therefore not clear whether this means that they possess better socio-cognitive skills than monkeys.

In addition to their close phylogenetic relationship to apes, baboons also live in large and complex groups. Given the advanced social capacities revealed through field experiments (see also Barrett 2009), they represent a promising species to explore their social and physicocognitive skills in a more controlled, laboratory setting. Nonetheless, systematic laboratory studies on baboon cognition, especially social cognition are relatively rare (see the physicocognitive studies by Fagot and colleagues in e.g. Fagot \& Paleressompoulle 2009; Fagot \& Thompson 2011). As close relatives of baboons, long-tailed macaques similarly provide interesting insights into primate cognitive evolution. With the exception of humans, macaques are the geographically most widely distributed primate genus (see Ostner 2006 for a review). Accordingly, they show a high degree of adaptability to different living conditions. Macaques are found in habitats ranging from the rain forests to snowy mountains to large Asian cities. Long-tailed macaques live in much smaller groups than baboons, but exhibit a number of interesting skills. For example, wild populations have been observed using stones to crack open oysters (Malaivijitnond et al. 2007b; Gumert et al. 2009). Hence, these monkeys possess toolusing abilities which have not yet received much attention. Furthermore, Reader and colleagues (2011) concluded from a meta-analysis that high general intelligence has independently evolved at least four times, with convergent evolution in capuchins, baboons, macaques and great apes. As a consequence of these facts, and the recently ambiguous results regarding ape and monkey cognition, in this thesis I have compared the skills of olive baboons and long-tailed macaques to that of apes by applying the complete suite of experiments of the Primate Cognition Test Battery (Herrmann et al. 2007), and added new paradigms and tests. Directly comparing the physico- and socio-cognitive skills of these species will provide new and interesting insights into the evolution of primate cognition. 


\subsection{Physical and Social Cognition}

"If ecological pressures have favoured the evolution of intelligence, then primates should be adept at solving ecological problems. Similarly, if social pressures have favoured the evolution of intelligence, then primates should be adept at solving social challenges."

Boyd \& Silk 2003, p. 235

This quotation summarizes why the assessment of both the physico- and socio-cognitive capacities of baboons and macaques in the present thesis is important. Comparing both cognitive domains helps to draw conclusion about the evolutionary pressures influencing cognition. I will now briefly describe the experiments I conducted with the goal of leading to a better understanding of primate cognition and why it may have evolved. In Study 1 (Chapter 2) I conducted the experiments of the Primate Cognition Test Battery, developed by Herrmann and colleagues (2007). This test battery relied on the division of physical and social cognition provided by Tomasello and Call (1997). They stated that physical cognition deals with inanimate objects and their spatial, numerical, and causal relations, whereas social cognition deals with other animate beings and their intentional actions, perceptions and knowledge.

All experiments of the PCTB conducted in the physical domain explore capacities that are ecologically relevant for the subjects, for example in a foraging situation. These included tests on spatial displacements, quantity discrimination and causality (the procedures of the single experiments are described in Chapter 2). Being able to locate objects in space, particularly food, is a prerequisite for successful foraging. In addition, tasks on object permanence (i.e. that objects continue to exist even when they are out of sight) and transpositions (where subjects have to follow the movement of objects) have also been conducted with other primate species and human children allowing for an even wider comparison of capacities within the primate lineages (compare Amici et al. 2010). Children, for example, seem to already have an understanding of object permanence with three months of age (Baillargeon \& DeVos 1991). Exploring these capacities in a wide range of primate species will help to determine their evolutionary roots.

The ability to judge quantities also has great relevance in a variety of ecological contexts, such as predation, foraging, and breeding (Dehaene 1997). This capacity has been widely explored in many animal species ranging from chickens to dogs, cats, fish and even insects (Ward \& Smuts 2007; Agrillo et al. 2008; Rugani et al. 2008; Carazo et al. 2009; Pisa \& Agrillo 2009; Agrillo et al. 2012). Furthermore, quantity discrimination is of great interest for neuroscience (see Nieder 2005 for a review), and brain studies have already revealed that specific neurons in the brain of monkeys respond to specific numbers (Nieder et al. 2002; Nieder et al. 2006; Tudusciuc \& Nieder 2007). The number of behavioural studies exploring the 
numerical capacities of monkeys is also constantly increasing (e.g. Beran 2007; Cantlon \& Brannon 2007; Addessi et al. 2008a; Beran 2008; Evans et al. 2010). Testing the quantity discrimination skills of olive baboons and long-tailed macaques therefore not only provides comparative data to elucidate the evolution of these skills, but also allows a closer examination of the underlying mechanisms (see Chapter 3 ).

Whether animals possess an understanding of causal mechanism has been extensively discussed (see Waldmann et al. 2006 for reviews; Penn \& Povinelli 2007). The aim of this thesis was, however, not to explore the details of causal understanding in monkeys, but whether the subjects understand the spatial-causal relationships between two objects (one of them being food) and to compare this capacity across different primate species. As mentioned earlier longtailed macaques have been observed using stones to extract food in the wild (Malaivijitnond et al. 2007b; Gumert et al. 2009), while olive baboons are not known to employ any tool using behaviour whatsoever. Comparing these closely related species in these kinds of tasks provides promising insights into the factors influencing the development of such skills.

Furthermore, I expanded the physico-cognitive experiments of the Primate Cognition Test Battery by examining apes' and monkeys' capacities to discriminate between differently sized objects (Chapter 4). The ability to discriminate between objects based on their size is very important for animals, as these regularly face the problem to distinguish between competitors, food items, or quality signals of different size. However, which factors influenced the evolution of fine-grained size discrimination abilities remains relatively unstudied. In this study we therefore tried to assess the possible influence of different socio-ecological and phylogenetic factors on the perceptual capabilities of primates (Matsuno \& Fujita 2009). Estimating differences in the perceptual abilities of animals is receiving increasing attention as researchers begin to acknowledge that these may have considerable influence on performances in cognitive tasks (Bshary et al. 2011).

In contrast to physical cognition, social cognition addresses capacities such as social learning, imitation, gaze following, and theory of mind. Such mechanisms form core elements of animal and human social behaviour (Fitch et al. 2010). In the course of my studies I therefore tested monkeys' abilities in social learning, using communicative cues and intention understanding (Chapter 2 and 5).

However, the problem with studies on social capacities is that it is unclear what exactly has been tested in the experiments. As mentioned earlier, it is possible that alternative explanations can account for the performance in the social tasks, which are mostly attributed to ample social cognition and an understanding of others intentions and mental states (see Tomasello et al. 
2003a). Perhaps subjects can solve the tasks of the Primate Cognition Test Battery using much more basal principles that do not need an understanding of other minds by applying simple physical knowledge. In the tests exploring the understanding of human pointing for example, the subjects may simply use physical principles of proximity between the hand of the experimenter and the correct cup to solve the task, without understanding her communicative intentions. To test this hypothesis we explored the macaques' comprehension of the human pointing cue in more detail (see Chapter 5).

\subsection{Aims and Approaches of this Thesis}

Taking the above mentioned aspects into account, I compared the cognitive abilities of baboons and macaques to those of great apes in a wide range of cognitive tasks, examining capacities of social and physical cognition, the so-called Primate Cognition Test Battery developed by Herrmann and colleagues (2007). The results of this study are reported in Chapter 2. This manuscript was recently accepted by PLOS ONE. To get a better understanding of the underlying factors that determined the species performances, I further investigated specific test paradigms of the PCTB and conducted additional experiments. In Chapter 3 and $4 \mathrm{I}$ focus on physical cognition; Chapter 5 expands on socio-cognitive capacities. Specifically, in Chapter 3 । present a study on the numerical competences of the monkeys, which assessed the influence of stimulus salience and representation on the monkeys' quantity discrimination abilities (published in Nature Communications). Chapter $\mathbf{4}$ summarizes a study on the size discrimination abilities of apes and monkeys, which we conducted in cooperation with Josep Call from the MPI for Evolutionary Anthropology in Leipzig. This manuscript is prepared for submission. Finally, Chapter $\mathbf{5}$ comprises a study on the socio-cognitive skills of monkeys; specifically we explored the long-tailed macaques' understanding of the human pointing gesture. We recently submitted this manuscript to a peer-reviewed journal on animal behaviour. In Chapter 6 I will summarize the results of my studies and discuss their implications for the ongoing examination of the evolution of cognition. 
Chapter 1 - General Introduction 
CHAPTER 2

Old World Monkeys Compare to Apes in the Primate Cognition

Test Battery

\author{
Vanessa Schmitt ${ }^{1,2}$, Birte Pankau ${ }^{1}$, Julia Fischer ${ }^{1,2}$ \\ ${ }^{1}$ Cognitive Ethology Lab, German Primate Center, Göttingen, Germany \\ ${ }^{2}$ Courant Research Centre Evolution of Social Behaviour, University of Göttingen, \\ Germany
}

PLOS ONE 7, e32024 (2012)

\title{
Author Contributions
}

VS and JF designed the study; VS and BP collected the data; VS analysed the data; VS and JF wrote the manuscript; all authors discussed the results and commented on the manuscript 


\title{
Chapter 2 - Monkeys Compare to Apes in the PCTB
}

\begin{abstract}
Understanding the evolution of intelligence rests on comparative analyses of brain sizes as well as the assessment of cognitive skills of different species in relation to potential selective pressures such as environmental conditions and social organization. Because of the strong interest in human cognition, much previous work has focused on the comparison of the cognitive skills of human toddlers to those of our closest living relatives, i.e. apes. Such analyses revealed that apes and children have relatively similar competencies in the physical domain, while human children excel in the socio-cognitive domain; in particular in terms of attention sharing, cooperation, and mental state attribution. To develop a full understanding of the evolutionary dynamics of primate intelligence, however, comparative data for monkeys are needed. We tested 18 Old World monkeys (long-tailed macaques and olive baboons) in the socalled Primate Cognition Test Battery (PCTB) (Herrmann et al. 2007, Science). Surprisingly, our tests revealed largely comparable results between Old World monkeys and the Great apes. Single comparisons showed that chimpanzees performed only better than the macaques in experiments on spatial understanding and tool use, but in none of the socio-cognitive tasks. These results question the clear-cut relationship between cognitive performance and brain size and - prima facie - support the view of an accelerated evolution of social intelligence in humans. One limitation, however, is that the initial experiments were devised to tap into human specific skills in the first place, thus potentially underestimating both true nonhuman primate competencies as well as species differences.
\end{abstract}




\section{Introduction}

Understanding the evolution of human cognition and communication rests primarily on comparative analyses with other extant members of the primate order. There are two major and interrelated streams of research; one focuses on the evolution of the brain, while the other aims at elucidating similarities and differences in behaviour. Such analyses thus incorporate information about the phylogenetic relationships between species as well as the putative selective pressures that might have played a role in shaping a species' cognitive skills. Within the hominoidea (apes and humans), the last common ancestor of humans and their closest relatives, the chimpanzees and bonobos, is dated at about 6 mya (Perelman et al. 2011), while the split between the Hominoidea and the Cercopithecoidea (Old World monkeys) occurred between 29 and 24 mya (Zalmout et al. 2010). Taking this phylogenetic information into account is a prerequisite for identifying the dynamics in the evolution of specific adaptations. One striking feature within the primate order is a disproportionate increase in relative brain size from monkeys to apes to humans (Jerison 1973). In particular, the neocortex has experienced considerable expansion. The neocortex is important for sensory perception, generation of motor commands, and higher cognition (Carlson 2007). In the 1980s, the most prominent hypothesis was that the increase in brain size in primates was related to frugivory, that is, the need to find food that is patchily distributed in space and time (Harvey et al. 1980). In recent years, the focus has returned to the idea that primate intelligence evolved in response to the challenges of living in large and complex groups - the so-called "Social Brain" hypothesis (Jolly 1972; Humphrey 1976; Byrne \& Whiten 1988; Dunbar 2003; Zuberbühler \& Byrne 2006; Byrne \& Bates 2010; Frith \& Frith 2010).

Whether this increase in brain size at the same time predicts an increase in cognitive abilities remains controversial. For instance, it has been proposed that overall brain size best predicts the cognitive abilities across nonhuman primates (Reader \& Laland 2002; Deaner et al. 2007). More recently, a number of scholars have aimed to derive more specific links between particular brain areas and cognitive performance. Shultz and Dunbar (2010), for example, claimed that the neocortex ratio and hippocampus volume are particularly important for problem solving and executive control. Others, however, have pointed out that attempts to link brain size to function is fraught with problems, including the choice of the variables entered in the analyses, and the problems associated with multiple correlations (Healy \& Rowe 2007). Further, size per se might not be the critical factor, but in fact the modularity and interconnectedness of different brain areas (Chittka \& Niven 2009). 


\section{Chapter 2 - Monkeys Compare to Apes in the PCTB}

Yet, it is undisputed that human brains are disproportionately larger than the brains of other primate species. In line with this, a systematic comparison of the cognitive skills of human toddlers and great apes revealed substantial differences in cognitive performance (Herrmann et al. 2007). Subjects were tested in largely identical experiments (the so-called Primate Cognition Test Battery [PCTB]). While great apes and children showed relatively similar competencies in the physical domain (space, quantities, causality), human children excelled in the sociocognitive tasks; in particular in terms of attention sharing, cooperation, and mental state attribution. This supports the assumption that social aspects were the driving force in the evolution of intelligence, at least in the transition from apes to humans.

To develop a full understanding of the evolutionary dynamics of primate intelligence, however, comparative data for monkeys are needed (MacLean et al. 2011). With the increase in brain size from monkeys to apes one would predict that apes would outperform monkeys in cognitive tasks. Indeed, Byrne and Whiten (1988) noted for example that tactical deception seems to be more common in great apes than in monkeys. Furthermore, only great apes recognize themselves in mirrors (Gallup 1982; Inoue-Nakamura 1997), lending further support for the distinction between monkeys and apes. A meta-analysis of published nonhuman primate cognition studies also indicated that "great apes significantly outperformed other lineages" ( $p$. 115) in their overall performance (2006).

In contrast to these results, a recent study by Amici and colleagues (2010) suggested that the cognitive abilities of monkeys and apes are not so different. They compared the performance of three monkey species (spider monkeys, capuchin monkeys, long-tailed macaques) and all four great ape species in spatial displacement and support tasks (i.e. using for example an unbroken cloth to pull in a reward) and found no support for a clear-cut difference between apes and monkeys. Notably, an additional analysis focusing on inhibition tasks revealed that species living in systems with fission-fusion dynamics (chimpanzees, bonobos, orangutans, and spider monkeys) outperformed members of species that live in more stable groups (long-tailed macaques, gorillas and capuchin monkeys). Apparently, the level of social complexity predicted the inhibitory skills better than phylogenetic relatedness or ecological conditions (Amici et al. 2008). One possible explanation for the discrepant assessments of the differences between monkeys and apes may be that the (meta-) analyses incorporated results of experiments or observations made in different studies using different methods. Furthermore, the differences between monkeys and apes may have been overestimated, because in many studies highly trained apes were compared to naive monkeys (Tomasello \& Call 1997). Thus, 
although more comparative studies are now available (Shettleworth 2009), systematic interspecific comparisons are still rare.

The differences in results may also be due to the fact that different tests may tap into different cognitive domains. In other words, there may be no increase in general intelligence from monkeys to apes, but more domain specific differences. Interestingly, Amici and colleagues (2010) found no clear distinction between monkeys and apes in their spatial memory, transposition, and support tasks, but what remains unknown is whether there are differences between the two lineages regarding other cognitive aspects. For instance, in the experiments by Herrmann et al. (Herrmann et al. 2007) great apes and children did not differ in their physico-cognitive capacities, but only in the experiments relying on social cognition. Perhaps this is also the case in the transition from monkeys to apes. Thus, we set out to systematically compare the skills of monkeys to that of apes, applying the same test as Hermann and colleagues on apes and toddlers. We therefore conducted the complete suite of experiments of the Primate Cognition Test Battery with Old World monkeys (olive baboons and long-tailed macaques) housed at the German Primate Center and compared them to the results of great apes. The data for the apes were kindly made available to us by Hermann and colleagues.

If an increase in brain size predicts an overall increase in cognitive performance, we would hypothesize that the monkeys perform less well than the apes in all experiments. In contrast, if an increase in brain size is (more or less) linearly related to an increase in socio-cognitive skills, then we would predict that the apes outcompete the monkeys especially in the socio-cognitive tasks, while they should perform on a more or less comparable level in the physical domain. However, it might also be the case that the human lineage underwent a nonlinear increase in socio-cognitive skills, in which case we would predict that apes and monkeys do not reveal substantial differences in either of the cognitive domains. As recent studies have shown further factors can influence the performance in cognitive tasks such as a shy or bold temperament (Herrmann et al. 2010; 2011) or the amount of inhibitory control (Amici et al. 2008; Schmitt \& Fischer 2011). To control for those aspects we included the temperament and inhibitory control experiments of Herrmann et al. (2007) in which we measured the subject's reaction to novel objects, people, and rewards, and their ability to control their impulses in a spatial memory task. In relation to the previous studies we expected to find an influence of these parameters on the cognitive performances of the monkeys. 


\section{MATERIAL AND METHODS}

\section{Ethics Statement}

All testing was non-invasive and the subjects participated voluntarily in the experiments. They were not food deprived for testing and water was always available ad libitum. All experiments were performed under the control of experienced veterinarians to ensure that the studies were in accordance with the NRC Guide for the Care and Use of Laboratory Animals and the European Directive 2010/63/EU on the protection of animals used for scientific purposes. Furthermore, in accordance with the German Animal Welfare Act and corresponding section for animals used for scientific purposes, the study approval was checked by the responsible Animal Welfare Officer of the German Primate Center (Permit Number 33.9-42502).

\section{Subjects}

We tested 13 long-tailed macaques (Macaca fascicularis) - 6 males and 7 females aged 1 to 7 years ( $M_{\text {age }}=2.8$ years) - living in a social group of 28 animals and 5 olive baboons (Papio anubis) -3 males and 2 females aged 3 to 9 years ( $M_{\text {age }}=6.1$ years) - living in a social group of 11 animals. The monkeys were housed at the German Primate Center in Göttingen and had access to indoor (baboons: 17sqm, macaques: 40sqm) and outdoor areas (baboons: 81sqm, macaques: 141sqm). The subjects were individually tested in their familiar indoor cages. Before the testing began all animals were trained to be separated from the group using positive reinforcement. One session lasted about 10 to 15 minutes. If an animal was not willing to participate in a session (e.g. not choosing a reward option) it was released again to the group and tested on another day.

\section{Primate Cognition Test Battery}

As the aim of this study was to conduct a systematic interspecific comparison, we used the same experimental procedures of the so-called Primate Cognition Test Battery (PCTB) as Herrmann and colleagues (2007). The PCTB consists of 16 tasks examining skills of physical cognition, i.e. an understanding of objects and their spatial, numeral and causal relationships, as well as of social cognition, i.e. an understanding of other animate beings and their intentional actions, perceptions, and knowledge. The 16 tasks are grouped into six scales. Three of these scales belong to the physical domain: Space, Quantity, and Causality. In these experiments we tested the monkeys' understanding of spatial displacements, their quantity discrimination abilities, and their understanding of the causal relations between two objects. The other three scales belong to the social domain: Social learning, Communication, and Theory of Mind. In these experiments we examined whether the monkeys imitate simple tasks such as shaking a 
reward out of a tube, understand communicative cues and intentional actions, as well as whether they follow the gaze of a human.

In contrast to Herrmann et al. we applied control conditions to some of the tasks and new quantity combinations in the quantity discrimination experiments (see File S1 for a detailed description of the methods). We adjusted the size of the material used to be operable for the baboons and long-tailed macaques, respectively. To facilitate the comparison of our results with those of Herrmann and colleagues, we here applied the same terminology as in the previous study. In the discussion, we will critically evaluate some of the connotations associated with the terms used for these experiments.

In the following we will shortly outline the experimental procedure of the 16 tasks of the PCTB. Some tasks consist of different items, which are described in detail in the Supporting Information and Herrmann et al (2007).

\section{Physical Domain}

Space: To test the monkeys' ability to track specific objects while they were being displaced in various ways, we conducted different 'spatial displacement' tasks. In total this scale is made up of four different tasks: Spatial Memory, Object Permanence, Rotation, and Transposition. In each task, three cups were aligned in a row on the testing tray and manipulated differently: To test their Spatial Memory two rewards were placed under two of the three cups and the subject was allowed to choose twice. In the Object Permanence task a small opaque cup, which contained a reward, was moved under one or two of the three larger cups in succession, leaving the reward under one of these at the end. The subject had to track these operations to locate the reward. We conducted an additional control condition in which the experimenter also touched the cups under which the smaller cup was not moved with her hand to examine whether the subjects only chose the last cup touched by the experimenter or really took into account where the smaller cup was moved to. In the Rotation task three cups, one containing the reward, were aligned on a moveable tray, which than was rotated $180^{\circ}$ and $360^{\circ}$. The subjects had to follow the rotation to locate the reward. In the Transposition task the position of the baited cup was switched with the position of the other cups in three different ways. The subjects had to follow these transpositions to locate the reward.

Quantities: To test the monkeys' abilities to discriminate between different food amounts, we conducted different two-choice experiments where they received the amount of food pieces they had pointed at. This scale consisted of two tasks: Relative Numbers and Addition Numbers. In the so-called Relative Numbers task the monkeys could choose between 1 and 8 food pieces lying on two different plates with differences between the two amounts ranging from 1 to 4 


\section{Chapter 2 - Monkeys Compare to Apes in the PCTB}

pieces. In the so-called Addition Numbers task the subjects were shown three different amounts of food items. The food items from the center plate were transferred to one of the side plates after a few seconds. The subjects had to choose the resulting larger number to be scored as a correct response.

Causality: To test their understanding of the spatial-causal relationships between two objects the monkeys were tested in four different tasks: Noise, Shape, Tool Use, and Tool Properties. In the Noise task, the subjects had to choose one of two cups. To give them a hint where the reward was located the cups were shaken. One cup contained a peanut and made a rattling sound when shaken. In the Shape task either two plastic boards or two pieces of cloth were placed on the tray. A reward was placed under one of the boards or cloths causing a visible bump, and the subjects were allowed to choose. To test their Tool Use abilities a reward was placed on the tray out of reach of the subject and a wooden stick was provided to the subject. The subject had to use the tool to retrieve the out of reach food. In the Tool Properties task a functional and a non-functional tool were presented. For example, a reward was placed on top of one piece of cloth, whereas the other reward was placed directly next to the other cloth piece. The subjects were allowed to pull one of the two pieces. Altogether, five different items were used in this task (cloth: food was placed on top or right next to a piece of cloth; Plexiglas bridge: a small bridge was placed over a piece of cloth; food was placed on top of the bridge or underneath directly onto the cloth; ripped cloth: food was placed on an intact or a ripped piece of cloth; broken wool: food was tied to the end of an intact or cut string of wool; tray circle: food was placed into a cardboard piece with a round hole in it or with a u-shaped opening, an attached string allowed the monkeys to pull the tray).

\section{Social Domain}

Social Learning: To test whether the monkeys' imitate simple actions done by a human to get food three different items were used. In all experiments a human demonstrator showed the subjects how to open three different plastic tubes which contained a reward (Paper tube, Banana tube, Stick tube). We scored whether the subjects solved the problem by the same means as the demonstrator. The behaviour of the subjects was compared to that of a control group ( 3 baboons and 3 macaques) who were given the opportunity to open the tubes without prior exposure to a human demonstrator (baseline condition).

Communication: To test their ability to use communicative cues by humans, the subjects were tested in three different tasks: Comprehension, Pointing Cups, and Attentional State.

The Comprehension task consisted of a two-choice paradigm in which the experimenter gave different cues to locate the reward. She either looked or pointed at the cup, which contained 
the reward or - in the control condition - placed an iconic marker (e.g. picture of a peanut) on it. The animal was then allowed to make its choice.

In the two tasks under the umbrella term Production two experimenters were needed. In the Pointing Cups task, one experimenter baited one of two cups, which were placed about 70 $\mathrm{cm}$ apart and left the room. Then the second experimenter entered the room. We then scored whether the subject indicated its choice by pointing at a cup. In the Attentional State task the attentional state of the main experimenter varied in four different ways. A second experimenter first placed a reward in front of the subject's cage and left the room. When the main experimenter entered the testing area, she either turned around and looked away from the reward, looked towards the reward, turned towards the reward but looked away or turned away from the reward but looked at it. The subject had to draw the experimenter's attention to the reward (e.g. by moving into her visual field and reaching for the reward) in order to receive it.

Theory of Mind: The experiments under this umbrella term encompassed experiments in two different tasks: Gaze Following and Intentions. In the Gaze Following task the experimenter sat in front of the monkey, hid a piece of food in her hands, and then completed three different actions: She held her hands in front of her body and looked up with her head and eyes; she sat with her back facing the subject, holding her hands next to her shoulders and looked up to the ceiling; or she held her hands in front of her body and glanced with her eyes only up to the ceiling. A response was scored if the subject followed the gaze of the experimenter and looked up. The behaviour of the subjects was compared to a baseline condition in which we measured how often the monkeys gazed upwards when the experimenter looked straight at the subject's chest (we did not stare directly at their eyes as this is a threatening behaviour in monkeys).

In the Intentions task, the experimenter tried to retrieve a reward out of one of two cups but failed. In the first test, she tried in vain to remove a lid; in the second test, a Plexiglas barrier blocked her access to the cup. The subject was then allowed to choose one of the cups.

\section{Testing Apparatus and General Procedure}

To test the cognitive capacities of the animals, they were separated from their group in their familiar indoor compartments. The testing apparatus used in most of the experiments (when other material was used it is indicated in the description of the experiments in the Supporting Information) consisted of a sliding board made of grey polyvinylchloride (length $0.8 \mathrm{~m}$, width $0.27 \mathrm{~m}$, height $0.01 \mathrm{~m}$ (baboons); length $0.55 \mathrm{~m}$, width $0.2 \mathrm{~m}$, height $0.01 \mathrm{~m}$ (macaques)), which was attached to a fixed polyvinylchloride table (length $0.8 \mathrm{~m}$, width $0.38 \mathrm{~m}$, height $0.01 \mathrm{~m}$ 


\section{Chapter 2 - Monkeys Compare to Apes in the PCTB}

(baboons); length $0.55 \mathrm{~m}$, width $0.3 \mathrm{~m}$, height $0.01 \mathrm{~m}$ (macaques)) by two drawer rails so that the sliding table could be moved horizontally. Three white opaque cups $(\varnothing 7.5 \mathrm{~cm} \times 7.5 \mathrm{~cm}$ height) or other materials (which are reported in File S1) were used to cover / present the food reward. These were placed on the sliding table. The sliding table was attached with an iron mount in front of a plastic panel. The middle of the plastic panel was cut out, which allowed one of two different kinds of plastic slices to be inserted, depending on the tasks performed. One of the plastic slices had three holes in it ( $\varnothing 1 \mathrm{~cm}$, distance $20 \mathrm{~cm}$ (baboons), distance $15 \mathrm{~cm}$ (macaques)) that allowed the subjects to point with their fingers at the cups. The other slice had two oval openings at the outer sites $(5.5 \mathrm{~cm} \times 2.5$, distance $25 \mathrm{~cm}$ (baboons), $5.5 \mathrm{~cm} \times 1.5 \mathrm{~cm}$, distance $30 \mathrm{~cm}$ (macaques)) to allow the subjects to retrieve e.g. pieces of cloth. Depending on the tasks, one of the two slices was attached to the panel.

Throughout testing, unless otherwise indicated, a choice was scored when the subjects pointed with one finger at one of the locations or put their fingers through one of the oval openings to retrieve e.g. cloth after the sliding table had been pushed against the Plexiglas panel. When the monkeys indicated the correct location, they were given a small food reward. However, unless otherwise stated, when they made incorrect responses they were always shown the location of the hidden food after each trial. The same desirable food items were used as rewards for most of the tasks (raisins, peanuts, pieces of fruits). It was possible to set up an occluder of grey plastic (length $0.8 \mathrm{~m}$, height $0.3 \mathrm{~cm}$, thickness $0.03 \mathrm{~m}$ ) in front of the panel so that the subjects were not able to watch the baiting procedures. All sessions were videotaped with a digital video camera (Sony DCR-HC90E). A naïve second observer coded $20 \%$ of all videotapes to assess inter-observer reliability, which was excellent (Cohen's $K=.97, N=809$ ).

\section{Design}

In general, we followed the design of Herrmann et al. (Herrmann et al. 2007) but doubled the number of trials in the object-choice tasks (see File S1) in order to include all possible spatial positions and combination of locations to control for the influence of using only a subset of all possible manipulations (see description of the specific tasks for details). Furthermore, we wanted to reduce the risk of obtaining significant effects by chance due to our smaller sample size in combination with a very small number of trials. We also controlled for learning over the trials, but did not find any effects in any of the tasks (Pearson Correlations between Trial and Performance; note however that only a small number of trials were conducted per condition and individual). For the other tasks, i.e. those that did not include object choice (Social learning, Attentional State and Gaze following), we applied the same number of trials as in the study by 
Herrmann and colleagues (2007) since in these experiments, the subjects had to perform specific actions, which probably did not happen coincidentally (see also control experiments for Social learning and Gaze following).

Furthermore, we pseudo-randomized and balanced the order of administering the experiments of the different scales across individuals to exclude any order effect. Eight of the individuals started with the experiments of the physical domain ( 5 baboons and 3 macaques) and ten started with the tasks of the social domain. There was no difference in the performance of the monkeys in relation to whether they started with the social or physical domain (ANOVA with first domain as between subject factor and performance in the two domains as dependent variables, $\left.F(2,15)=0.28 p=.757, \eta^{2}=.036\right)$. Within each scale and task, the order of the experiments was also pseudo-randomized and balanced across individuals.

All macaques were completely naive to cognitive testing and working with a human experimenter prior to these experiments. The baboons had already participated in an experiment on size discrimination (manuscript in preparation) and a study about inferential reasoning (Schmitt \& Fischer 2009).

\section{Influence of Temperament, Inhibitory Control and Rank on Performance}

To test whether differing temperaments, inhibitory control or rank positions influenced the monkeys' performances in the test battery, each subject participated in a set of additional tests comparable to those used by Herrmann et al. (2007) (see File S2 for detailed descriptions). In terms of temperament we measured the subjects approaching behaviour to novel objects, people and foods. Their amount of inhibitory control was examined during an additional spatial memory task, which specifically assessed whether the monkeys are able to skip the middle out of three cups. To assess the influence of rank we classified each individual as high, middle or low ranking on the basis of focal observations done by V.S..We then tested whether the results of these measurements correlated with the performance of the monkeys in the PCTB (Pearson correlations).

\section{Data analysis}

First, we determined the overall proportion of correct responses in each task for every subject. To measure whether the baboons and macaques performed above chance level or baseline we used the Wilcoxon-test because of the small sample sizes (to correct for multiple testing we applied a Benjamini-Hochberg correction). On the individual level we used Binomialtests to see whether the performance was significantly better than expected by chance. To explore whether there were significant differences between the performances of the baboons 


\section{Chapter 2 - Monkeys Compare to Apes in the PCTB}

and macaques in the test battery we conducted multivariate analysis of variance (MANOVA) with species and sex or rank as between-subject factor and performance of the baboons and macaques in each task as dependent variable. In case of significant effects we controlled for age by using analysis of covariance (ANCOVA). To further compare the performance of the baboons and macaques in each task we used univariate analysis of Variance (ANOVA) or in cases when data were not normally distributed (normality test using Shapiro Wilks-tests) a Kruskal-Wallis ANOVA. In case of significant effects, post-hoc tests (Bonferroni) were conducted. For the tool use task no statistical analyses were possible as performance was zero for all subjects. As we did not have information about the performance of the apes in each task, we only conducted repeated measures ANOVAs on the scale level, with follow-up post-hoc tests (Bonferroni) to compare the performance of the four species (baboon, macaque, chimpanzee, orangutan). The critical p-value was set to $\alpha=.05$ (except for pair wise comparisons) and all tests were twotailed.

\section{RESULTS}

\section{Performance in the different Tasks}

Space: On the species level both baboons and macaques performed significantly above chance level (0.33) in all four tasks of the scale Space (see Figure 1) (macaques: Spatial Memory $(z=3.04$, adjusted $p=.012)$, Object Permanence $(z=3.18$, adjusted $p=.022)$, Rotation $(z=3.18$ adjusted $p=.015)$; and Transposition $(z=2.76$, adjusted $p=.016)$; baboons: Spatial Memory, Object Permanence, Rotation and Transposition (all $z s=2.02$, all adjusted $p s=.049$ )). The macaques also performed above chance level in the control task we conducted during the Object Permanence tests $(z=2.93$, adjusted $p=.012)$, and the performance of the baboons also nearly reached significance $(z=1.83$, adjusted $p=.075)$. On the individual level, however, none of the macaques performed above chance in the transposition task, whereas four out of five baboons did (see Table 1).

Quantity: Both species performed significantly above chance level $(0.5)$ in the two tasks on quantity discrimination (see Figure 1 ) (macaques: Relative Numbers $(z=3.06$, adjusted $p=.013$ ), Addition Numbers $(z=2.80$, adjusted $p=.017)$; baboons: Relative Numbers $(z=2.02$, adjusted $p$ $=.049)$, Addition Numbers $(z=2.02$, adjusted $p=.049))$. On the individual level none of the baboons, but two macaques performed above chance in the Addition Number task (see Table 1).

Causality: None of the baboons or macaques solved the tool use task where they had to use a stick to retrieve food. However, on the species level the macaques performed above chance 
(0.5) in two other tasks of the scale Causality: Shape $(z=2.67$, adjusted $p=.019)$, and Tool properties $(z=3.18$, adjusted $p=.044)$ and nearly reached significant values in the task Noise $(z$ $=2.20$, adjusted $p=.055$ ) (see Figure 1 ). The baboons however only performed significantly above chance in the Shape condition ( $z=2.02$, adjusted $p=.049$ ).

Social learning: In the baseline condition, where the subjects did not get any demonstration on how to open the different tubes, none of the six subjects used a method similar to the one demonstrated in the test condition. In this condition, neither the baboons nor the macaques showed any evidence of social learning. Only once did one baboon use a similar technique as the human demonstrator, but that does not deviate significantly from the baseline (0.0) (see Figure 1).

Communication: The macaques performed significantly above chance level in all three tasks of the scale Communication, i.e. Comprehension (chance $0.5, z=2.83$, adjusted $p=.014$ ), Pointing Cups (chance 0.5, $z=2.93$, adjusted $p=.012$ ), and Attentional State (chance $0, z=2.37$, adjusted $p=.038$ ). The baboons performed significantly above chance only in the Comprehension ( $z=2.02$, adjusted $p=.049)$ and Pointing Cups tasks $(z=2.02$, adjusted $p=$ .049), but not in the Attentional State condition ( $z=1.60$, adjusted $p=.113$ ) (see Figure 1 ). However, none of the baboons performed above chance in the Gaze and Point condition of the Comprehension task, but three macaques did (Binomial-tests, $p=.016$ ). In contrast, none of the macaques performed significantly above chance in the Attentional state task (see Table 1), whereas two of the baboons scored a $100 \%$ correct.

Theory of Mind: Considering gaze following both baboons and macaques performed significantly above baseline, which we assessed by the monkeys' looks upwards while the experimenter was looking straight (they looked upwards in $M=10$ percent of all trials). In the test situation the macaques followed the human gaze very often $(\mathrm{M}=.57, z=3.18$, adjusted $p=$ .011 ), whereas the baboons did so a bit less ( $M=.33, z=2.02$, adjusted $p=.049)$ (see Figure 1 ). Both species also performed significantly above chance level $(0.5)$ in the task on understanding intentions (macaques: $z=2.52$, adjusted $p=.027$; baboons: $z=2.02$, adjusted $p=.049$ ). On the individual level 12 macaques and three baboons followed the human gaze significantly more often than in the baseline condition, whereas only two out of 13 macaques performed above chance in the Intention task, but three out of five baboons did (see Table 1). 


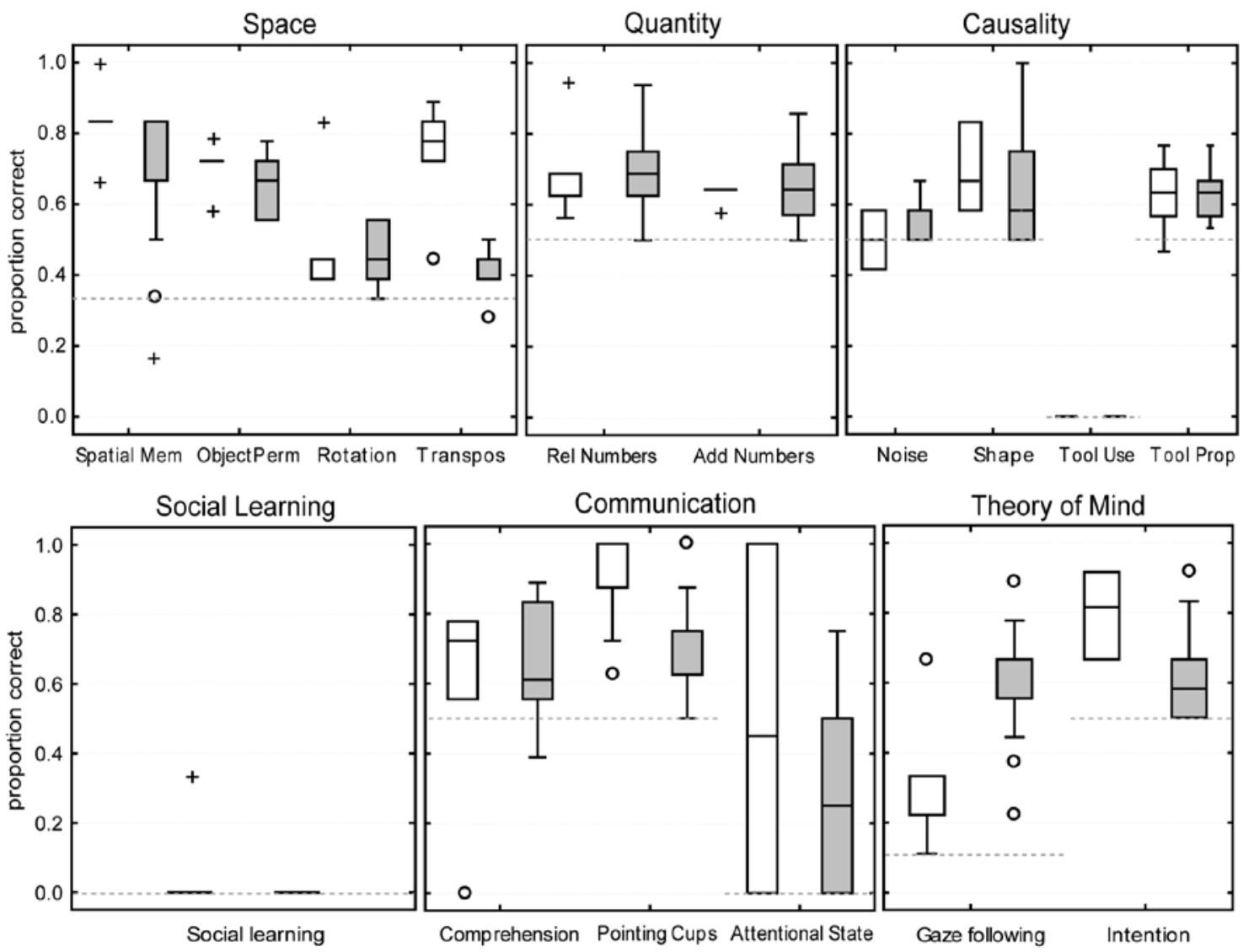

Figure 1. Performance of the monkeys in the PCTB. Shown are the proportions of correct responses of the baboons (white) and macaques (grey) in the 16 tasks of the PCTB grouped into the respective scale. Boxes show the interquartile range from the $25^{\text {th }}$ to the $75^{\text {th }}$ percentile. The line across the boxes represents the median. The whiskers indicate the maximum and minimum values excluding outliers (circles) and extreme values (crosses). The dotted lines represent the chance level and baseline, respectively for each task.

\section{Comparison of Baboons and Macaques}

As none of the baboons or macaques solved the tool use task, we had to exclude it from the following statistical analysis of variance. Considering the performance in the other 15 tasks of the PCTB a multivariate analysis of variance revealed no significant differences between the two species (MANOVA with species and sex as between-subject factor and performance in the 15 different tasks as dependent variables; Wilk's Lambda, $\left.F(11,1)=4.88, p=.346, \eta^{2}=.982\right)$. However, as Figure 1 indicates, there was a large difference between the species in the transposition task, and univariate analyses indeed revealed that here the baboons performed significantly better than the macaques (Kruskal-Wallis ANOVA, $H(1, N=18)=8.10, p=.004$ ) also when age was controlled for $\left(F(1,15)=119.61, p<.001, \eta^{2}=.889\right)$. There were no significant differences between the species in any other tasks after correction for multiple testing (all ps $>.01$ ). 


\section{Influence of Temperament, Inhibitory Control and Rank}

We found no significant correlations between the temperament measures and the performance of the monkeys in the two domains for either macaques (social domain $(r(12)=-$ $.27, p=.395)$, physical domain ( $r(12)=.18, p=.576)$; nor baboons (social domain $(r(5)=.66, p=$ .229), physical domain ( $r(5)=.58, p=.299)$. Furthermore, there were no significant correlations between the performance in the social and physical domain and the inhibitory control task for the macaques (social domain: Spearman correlations $(13)=-.18, p=.565$; physical domain: Spearman correlations $(13)=.04, p=.892$ ) and baboons (social domain: Spearman correlations $(5)=.58, p=.308$; physical domain: Spearman correlations $(5)=.00, p=1)$. Rank and sex had also no effect on the performance of the monkeys in the PCTB (rank: $F(18,2)=1.84, p=.409, \eta^{2}$ $\left.=.943 ; \operatorname{sex}: F(11,1)=2.15, p=.491, \eta^{2}=.959\right)$.

\section{Comparison of Monkeys and Apes}

To compare the performances of the four species (baboons, macaques, chimpanzees, orangutans) we calculated the mean proportion of correct responses in each scale for the two monkey species and compared this to the results for the chimpanzees and orangutans taken from Herrmann et al. (2007) (Figure 2). Statistical analysis revealed a significant effect of species (repeated measures ANOVA; Wilk' Lambda, $F(18,407.78)=6.09, p<.001, \eta^{2}=.211$ ). Post-hoc test (Bonferroni), however, showed that there were no significant differences between monkeys and apes in the scales Quantity, Social learning and Communication (all ps >.266). The chimpanzees performed significantly better than the macaques only in the scales Space and Causality (Post-hoc tests, space $p<.001$, causality $p<.001$ ) and better than the baboons only in the scale Causality (Post-hoc test, $p=.005$ ). However, the differences in the scale Causality were mainly due to the 'tool use' task, which none of the monkeys solved. Looking at the scale Causality without including the tool use task there were no significant differences between the species (Posthoc tests, $p=1$ ). In the scale Theory of Mind the macaques performed significantly better than the chimpanzees $(p<.001)$ and orangutans $(p<.001)$, and the baboons performed significantly better than the orangutans $(p=.002)$. There were no significant differences between the baboons and chimpanzees after correction for multiple testing in this scale. 

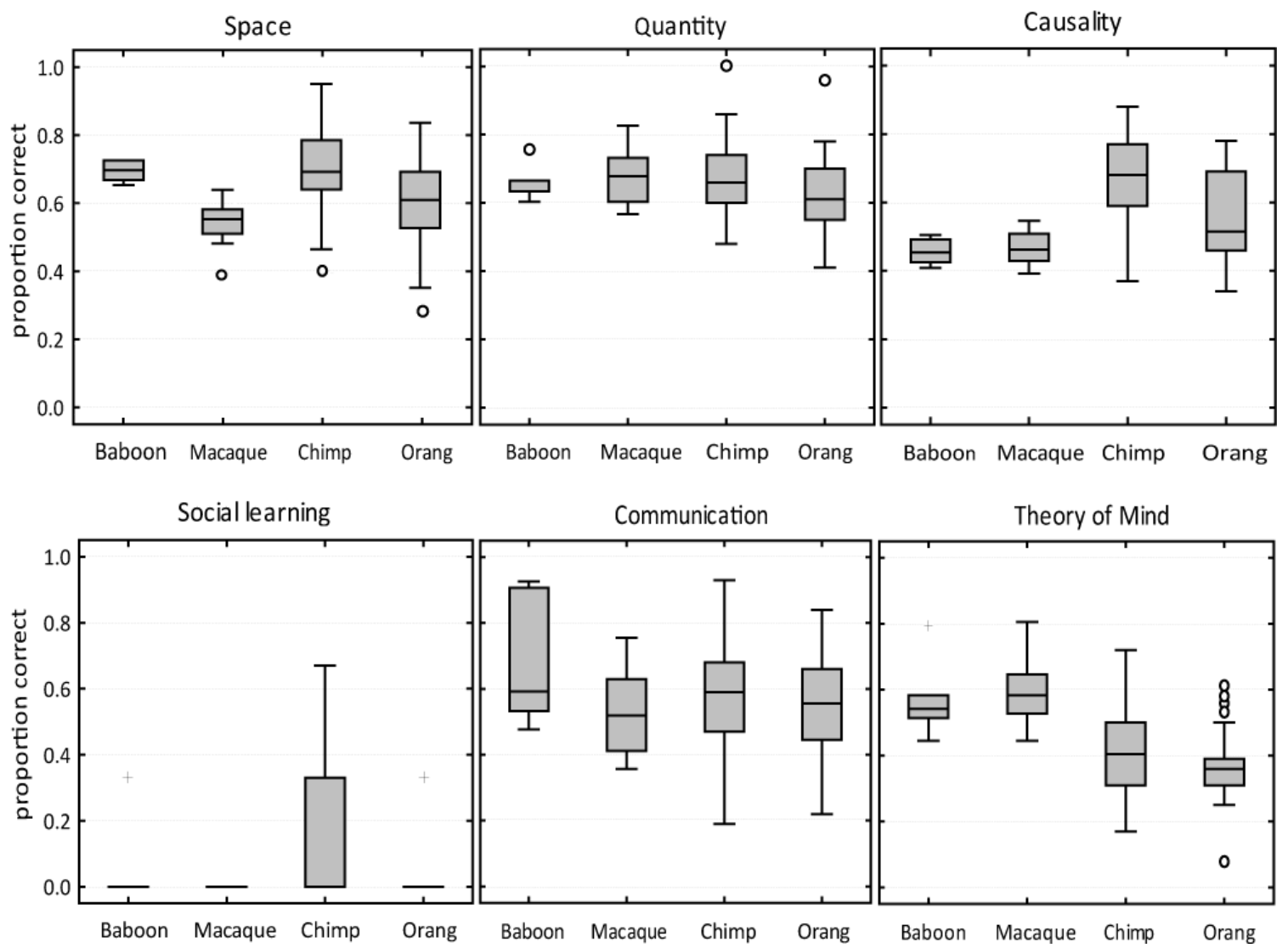

Figure 2. Comparison of species. Shown are the proportions of correct responses on the scale level for the four different primate species. Boxes show the interquartile range from the $25^{\text {th }}$ to the $75^{\text {th }}$ percentile. The line across the boxes represents the median. The whiskers indicate the maximum and minimum values excluding outliers (circles) and extreme values (crosses).

\section{DISCUSSION}

The results of our experiments indicate that olive baboons and long-tailed macaques have a very good understanding of objects and their spatial, numeral, and causal relations. Both monkey species performed above chance in all tasks on spatial displacements and quantity discrimination and showed only some limitations in experiments on causal understanding (e.g. tool use). An analysis of the performance in the social domain reveals a more inconsistent picture. Although the monkeys followed the gaze of the human experimenter significantly more often than in the baseline condition, they only marginally used the gazing cue in the objectchoice task. Furthermore, members of both species did not show any indication of imitation in the social learning tests and only two baboons seemed to show some understanding of the attentional state of the experimenter. In contrast the monkeys performed very well when intentional actions of the experimenter served as a cue in an object choice task. However, as this can also be explained by simply using local enhancement, this result should be interpreted with caution (see below). 
The good performances of our monkeys in the physical domain are in line with a recent comparative study on New and Old World monkeys, which found no clear-cut distinction between the capacities of monkeys and apes (Amici et al. 2010). Comparing the performance of our monkeys to that of great apes in the physical tasks of the PCTB also revealed no distinct differences between the two taxa (i.e. Hominoidea and Cercopithecoidea). However, we found cognitive differences between particular species, especially in tasks on spatial transpositions. The olive baboons in our experiments outperformed all three monkey species (spider monkeys, capuchin monkeys, long-tailed macaques) tested by Amici and colleagues (2010), mirroring our finding that the baboons performed significantly better than the macaques in the Transposition task. This is particularly interesting as mastering these fairly demanding object displacement tasks has not yet been reliably shown in Old World monkeys and the ability has long been used as the main type of data to support the distinction between apes and monkeys (Deaner et al. 2006). We, however, found no significant differences between the performances of baboons and great apes in these tasks. Interestingly Herrmann et al. (2007) also found significant differences in the scale Space between chimpanzees and orangutans. Thus, although there are differences between species in this aspect, there seems to be no deep split between apes and monkeys. Recently, differences in cognitive capacity have been linked to social organisation. Specifically, it was suggested that subjects living in fission-fusion societies may exhibit enhanced cognitive skills (Amici et al. 2008, 2010). Constant fission and fusion among subgroups is thought to require enhanced memory, inhibitory control and analogical reasoning as subjects are permanently confronted with changing group compositions (Barrett et al. 2003). For our two species, however, this explanation does not apply as both olive baboons and long-tailed macaques live in stable female-bonded groups. Why certain species do better than others in spatial tasks may also have something to do with the foraging techniques used, but this issue requires further empirical investigation.

Furthermore, we found no differences between monkeys and apes in the quantity discrimination tasks, and the differences in the scale Causality were mainly due to the monkeys failing to use a stick to retrieve out-of-reach food. In contrast to the finding by Amici and colleagues (2010), however, our long-tailed macaques performed reasonably well in the tasks on tool properties. Indeed, long-tailed macaques have been reported to use tools in the wild, supporting the assumption that they should have some understanding of the causal relations between an object and food. For instance, they use stones to crack open oysters or crabs and do so quite efficiently (Malaivijitnond et al. 2007b; Gumert et al. 2009). That they failed in the Tool Use task of the PCTB may therefore be due to the high difficulty of this task as it requires 


\section{Chapter 2 - Monkeys Compare to Apes in the PCTB}

quite fine scaled motor control and may have had too little ecological relevance for the monkeys. These results further support the view that the physico-cognitive capacities of monkeys and apes are not that distinct in general, but that differences between species exist in more specific aspects, which may be better explained by socio-ecological aspects than by phylogenetic relationships (Amici et al. 2010).

Concerning the tasks of the social domain, we also did not find an increase in performance from monkeys to apes. The long-tailed macaques even scored significantly higher than the apes in the Theory of Mind scale. However, despite the fact that the monkeys did well in the Theory of Mind tasks, it should be noted that most of the tasks can be solved without attribution of mental states. For instance, gaze following can be conceived as a simple orienting reflex or somewhat more elaborate as behaviour reading (for a study on gaze following see Teufel et al. 2010). Thus, the extensive gaze-following behaviour of the macaques does not imply an enhanced understanding of other minds, especially in comparison to the baboons and apes. The macaques seemed to be more re-active and tuned to the experimenter during the gaze following experiments, probably leading to a slowed habituation.

The Pointing Cups and Intention tasks also consisted of two-choice tests, which could have been solved by just behaviour reading or by using spatial associations as e.g. the proximity between the experimenter's hand and the cup (for a discussion on behaviour reading see Emery \& Clayton 2009; Shettleworth 2010a). Furthermore, the baboons had already participated in two-choice experiments before and it may well be that their former experience with human subjects in this kind of setup improved their performance. To summarize, we do not imply that our subjects attribute mental states to others, and only chose this terminology to remain consistent with previous studies (Herrmann et al. 2007).

Taken together, our experiments neither showed an increase in general intelligence nor in socio-cognitive abilities from Old World monkeys to apes, contradicting the theory that an increase in brain size is necessarily linked to an increase in intelligence (Deaner et al. 2006). In contrast, the species differences we found were on a more domain specific level (for example spatial displacements) with variation between but also within taxa. These findings may be somewhat surprising as a number of studies claimed that there is a large difference between apes and monkeys, in particular in their ability to form mental representations (Byrne 2000), i.e. to hold in mind and operate on mental objects that have semantic properties (Pitt 2008). Whereas the cognitive system of great apes was interpreted as qualitatively more human-like with some understanding of others' mental states, desires, and intentions (Tomasello et al. 2003b, a; Call et al. 2004), the cognitive abilities shown by monkeys were mainly attributed to 
rapid learning capacities (Byrne 1997; 2000). However, a recent study suggests that not only apes but also monkeys are able to form mental representations (Schmitt \& Fischer 2011). We tested the same olive baboons and long-tailed macaques in a quantity discrimination task with food and non-food items and found that the performance of the monkeys was influenced by their representation of the items as reward or choice stimuli and not by their quality (being edible or not).

Yet, we do not claim that the cognitive abilities of monkeys and apes are generally similar, either. It could also be the case that only the cognitive competencies in the items that were tested in the PCTB do not differ substantially. The PCTB mainly consists of experiments from developmental psychology that were designed to unravel the ontogeny of human specific skills (Social learning, Communication, Theory of Mind). Thus, it is possible that the tasks have been too difficult to allow a measurable difference between monkeys and apes. On the other hand, the good performance in the physical domain may constitute a ceiling effect. In other words, these tasks were structurally simpler and thus yielded high scores in many of the tasks. Furthermore, although the monkeys were able to solve most of the tasks in the physical domain, it is not clear whether they really had an understanding of the underlying physical properties. So it may well be that one would find differences between species when analyzing more specifically how the subjects solved the different tasks (Call 2007).

Another issue that needs to be evaluated critically is the fact that in the original study (Herrmann et al. 2007), two to three year old children were compared to mainly adult apes and monkeys, respectively. It would be highly desirable to assess the performance of adult humans in these tasks to obtain a more comprehensive picture. Moreover, in contrast to nonhuman primates, children are tested by members of their own species (for a critical review see Boesch 2007). Thus, such species comparisons often cannot control for a number of serious confounds, which should be held in mind.

Despite these limitations, the idea to test different species in such a large battery of tasks is a productive approach in comparative cognition studies. There is a caveat, however. As Tinbergen (Tinbergen 1951) already pointed out, the same test for a different species may not be the same test. Above all, this applies to situations where a given test yields different results (as in the case of the children vs. the nonhuman primates). In such instances, it is necessary to further investigate why a given species apparently fails in a certain test, and to develop experiments with a high ecological validity for each species. For instance, baboons and macaques hardly ever use sticks to retrieve food, so it is perhaps not surprising that they failed in this task. 


\section{Chapter 2 - Monkeys Compare to Apes in the PCTB}

A recent paper on comparative phylogenetic methods strongly encourages the integration of comparative psychology and evolutionary biology (MacLean et al. 2011). It is particularly important to consider variation in the species' socio-ecology in such analyses. Furthermore, future studies should also take care of variations in physiological characteristics between species, as e.g. in visual fields or attention patterns. Differences between species may be influenced by perceptual rather than cognitive differences. In addition, it would be highly desirable to compare the abilities of the same species in different labs and settings, to obtain a comprehensive understanding of the variability between and within species. Although we did not find any significant correlations between the temperament and inhibition control measures respectively and the cognitive performance of the monkeys in our study, taking such additional factors into account has proven to be useful when comparing the cognitive abilities of different species (Herrmann et al. 2007; Amici et al. 2008).

In conclusion, our study provides the first evidence that the cognitive skills of monkeys and apes are much more similar than expected both in the social and physical domain, at least in the tests of the РСТВ. Hence, our results support the view of an accelerated evolution of social respectively cultural intelligence in humans (Herrmann et al. 2007). We could furthermore show that it is essential to use a wide range of experiments when comparing the cognitive capacities of different species. Using only a subset of experiments (e.g. only spatial displacements) would have led to completely different conclusions. Thus, future comparative approaches should also consider including multiple cognitive experiments of different domains.

\section{ACKNOWLEDGMENTS}

First of all we would like to thank Esther Herrmann and her colleagues for providing us with the data for the chimpanzees and orangutans performances in the PCTB, which were already published in their study in 2007. We are furthermore indebted to Uwe Schönmann and Annette Husung and all the animal caretakers for their help.

\section{FUNDING}

This study was funded by the German Initiative of Excellence and the Leibniz Graduate School for Primate Neurobiology (Neuroprim). The funders had no role in study design, data collection and analysis, decision to publish, or preparation of the manuscript. 
Table 1: Mean proportion of correct responses of the baboons and macaques in each task (and Scale) of the PCTB.

\begin{tabular}{|c|c|c|c|c|c|c|c|c|c|c|}
\hline & & ma & aques & & & & boons & & & \\
\hline Tasks & Trials & $\mathrm{n}$ & M (SD) & $95 \% \mathrm{Cl}$ & Ind & $n$ & $M(S D)$ & $95 \% \mathrm{Cl}$ & Ind & Chance \\
\hline Space & & & $.54(.06)$ & {$[.51, .58]$} & & & $.69(.03)$ & {$[.65, .73]$} & & \\
\hline Spatial Mem & 6 & 13 & $.68(.22)$ & {$[.55, .81]$} & 7 & 5 & $.83(.12)$ & {$[.69, .98]$} & 4 & .33 \\
\hline Object Perm & 18 & 13 & $.65(.08)$ & {$[.60, .70]$} & 13 & 5 & $.71(.06)$ & {$[.64, .79]$} & 5 & .33 \\
\hline Rotation & 18 & 13 & $.46(.09)$ & {$[.41, .51]$} & 5 & 5 & $.49(.19)$ & {$[.25, .73]$} & 1 & .33 \\
\hline Transposition & 18 & 13 & $.39(.07)$ & {$[.35, .44]$} & 0 & 5 & $.73(.17)$ & {$[.52, .95]$} & 4 & .33 \\
\hline Quantity & & & $.67(.08)$ & {$[.62, .72]$} & & & $.66(.06)$ & {$[.59, .73]$} & & \\
\hline Rel Numbers & 16 & 13 & $.70(.13)$ & {$[.62, .78]$} & 4 & 5 & $.69(.15)$ & {$[.51, .87]$} & 1 & .50 \\
\hline Add Numbers & 14 & 12 & $.64(.11)$ & {$[.57, .71]$} & 2 & 5 & $.63(.03)$ & {$[.59, .67]$} & 0 & .50 \\
\hline Causality & & & $.46(.05)$ & {$[.43, .50]$} & & & $.46(.04)$ & {$[.41, .51]$} & & \\
\hline Noise & 12 & 13 & $.56(.07)$ & {$[.51, .60]$} & 0 & 5 & $.50(.08)$ & {$[.40, .60]$} & 0 & .50 \\
\hline Shape & 12 & 13 & $.66(.17)$ & {$[.56, .76]$} & 2 & 5 & $.70(.13)$ & {$[.54, .86]$} & 2 & .50 \\
\hline Tool Use & 1 & 13 & 0 & & 0 & 5 & 0 & & 0 & .00 \\
\hline Tool Prop & 30 & 13 & $.64(.08)$ & {$[.59, .68]$} & 4 & 5 & $.63(.12)$ & {$[.48, .77]$} & 2 & .50 \\
\hline Social learning & 3 & 10 & 0 & & 0 & 5 & $.07(.15)$ & {$[-.12, .25]$} & 0 & $.00^{a}$ \\
\hline Communication & & & $.53(.13)$ & {$[.45, .61]$} & & & $.69(.21)$ & {$[.42, .95]$} & & \\
\hline Comprehension & 18 & 13 & $.66(.17)$ & {$[.56, .76]$} & 4 & 5 & $.71(.09)$ & {$[.60, .82]$} & 4 & .50 \\
\hline Pointing Cups & 8 & 13 & $.69(.15)$ & {$[.60, .78]$} & 3 & 5 & $.90(.16)$ & {$[.70,1.10]$} & 4 & .50 \\
\hline Attention State & 4 & 13 & $.23(.26)$ & {$[.07, .39]$} & 0 & 5 & $.45(.51)$ & {$[-.19,1.09]$} & 2 & .00 \\
\hline Theory of Mind & & & $.59(.10)$ & {$[.53, .65]$} & & & $.57(.13)$ & {$[.41, .74]$} & & \\
\hline Gaze following & 9 & 13 & $.57(.17)$ & {$[.47, .67]$} & 12 & 5 & $.33(.21)$ & {$[.08, .60]$} & 3 & $.10^{\mathrm{a}}$ \\
\hline Intention & 12 & 13 & $.62(.14)$ & {$[.53, .70]$} & 2 & 5 & $.82(.11)$ & {$[.68, .95]$} & 3 & .50 \\
\hline
\end{tabular}

Note: Significant deviations from chance level are in boldface $(\alpha=.05)$. Performance on the scale level was not compared to chance as this varies between tasks. Trials $=$ Number of trials performed in each task; $\mathbf{n}=$ Number of tested individuals; Ind = Number of individuals performing above chance level; $\mathbf{C l}=$ confidence interval; ${ }^{a}$ results of the baseline conditions 


\section{SUPPORTING INFORMATION}

File S1: Detailed description of the experiments used in the PCTB

File S2: Detailed description of the methods used to assess temperament, inhibitory control and rank

\section{File S1}

\section{Supporting Information on Methods of the PCTB}

\section{Primate Cognition Test Battery}

The tasks and procedures were adopted from the study by Herrmann et al. (2007). When we did changes in the experimental procedure these are marked in bold and italic face. Otherwise the methods used were the same (therefore we also used similar wording to describe the tasks in order to avoid confusion) and only the size of the material was adjusted to be operable for the baboons and long-tailed macaques, respectively.

\section{Physical Domain}

1. Space

\section{a. Spatial Memory}

Three cups were placed in a row on the platform in front of the testing cage. The experimenter then showed the subject two rewards and placed them under two of the three cups in full view of the subject. Then the platform was pushed towards the subject and it was allowed to make up to two choices in succession. If, however, the subject chose the empty cup first, it was not allowed to make further choices. The response was counted as correct when the subject had chosen both baited cups in succession.

\section{b. Object Permanence}

Three cups were placed in a row in front of the testing cage. An additional small opaque cup was placed on the far left or far right side, respectively of the platform. The experimenter placed a reward under this small cup while the subject was watching. The small cup was then moved towards one of the larger cups, which was slightly lifted by raising the side not facing the subject. The experimenter then made a swapping movement with the small cup as if swapping the reward under the larger cup. There were three possible displacements performed:

Single displacement: The experimenter moved the small cup hiding the reward under one of the three cups as described above, swapped the reward under it and did not touch the other two cups.

Double adjacent displacement: The experimenter moved the small cup hiding the reward under two adjacent cups in succession as described above, left the reward under one of these cups without touching the third cup.

Double non-adjacent displacement: The experimenter moved the small cup hiding the reward under the left and right cup in succession as described above and left the reward under one of them. The cup standing in the middle was not touched.

Single displacement touch: The experimenter moved the small cup hiding the reward under one of the three cups as described above and left the reward under this cup. However, in this condition the experimenter touched the other two cups with her hand to examine whether the 
subjects only chose the last cup touched by the experimenter or really took into account where the smaller cup was moved to.

After moving the small under the specific larger cups the experimenter lifted the small cup to show the monkey that the small cup was now empty. The platform was pushed forward and the monkey was allowed to choose either one cup (in the single displacement item and control condition) or up to two cups (in the double displacement items). However, if the subject chose a cup under which the smaller cup was not moved during the demonstration, no further choices were allowed. A correct response was counted when the monkey had chosen the baited cup before choosing a cup which was not manipulated at all.

\section{c. Rotation}

Three cups were placed in a row on a tray, which was then placed on the platform in front of the testing cage. The experimenter showed a reward to the monkey and placed it under one of the three cups while the subject was watching. Then the tray was rotated in three possible ways:

$180^{\circ}$ middle: The reward was placed under the middle cup, and the tray was rotated $180^{\circ}$ in clockwise or counterclockwise direction, respectively. After the rotation, the reward was located in the same location as it was initially placed.

$360^{\circ}$ : The reward was placed under either the left or right cup, and the tray was rotated $360^{\circ}$ in clockwise or counterclockwise direction, respectively. After the rotation, the reward was located in the same location as it was initially placed.

$180^{\circ}$ side: The reward was placed under either the left or right cup, and the tray was rotated $180^{\circ}$ in clockwise or counterclockwise direction, respectively. After the rotation, the reward was located on the opposite side of where it was initially placed.

After the completed rotation the subject was allowed to choose one cup. A correct response was scored when the subject chose the baited cup first.

\section{d. Transposition}

Three cups were placed in a row on the platform in front of the monkeys' testing cage. The experimenter showed a reward to the monkey and placed it under one of the three cups while the subject was watching. Then one of three possible manipulations was performed:

Single transposition: The experimenter switched the position of the baited cup with one of the empty cups. The third cup was not touched.

Double unbaited transposition: The experimenter switched the position of the baited cup with one of the empty cups. Then the positions of the two empty cups were switched.

Double baited transposition: The experimenter switched the position of the baited cup with one of the empty cups. Then she switched the position of the baited cup again with one of the empty cups.

After the transpositions were completed the subject was allowed to choose one cup. A correct response was scored if the monkey chose the baited cup first.

\section{Quantities}

\section{a. Relative Numbers}

The experimenter placed two plates on the platform in front of the testing cage and put up an occluder to prevent the monkeys from watching the baiting procedure. Then she baited the plates with different amounts of equal sized food pieces (half a peanut or raisin was used as a unit). The experimenter then placed the plates in the middle on the platform and removed the 
occluder so the subjects could see the amounts lying on each plate. After $\sim 5$ seconds had passed and the subject paid attention, the experimenter moved the plates simultaneously to the sides of the platform, one to the right and one to the left. The sliding table was pushed against the Plexiglas panel and the subject was allowed to choose and received all food pieces lying on the respective plate. Each subject received one trial for each of the following pairs of numbers (the order was randomized):

1:0, 1:2, 1:3, 1:4, 1:5, 2:3, 2:4, 2:5, 2:6, 3:4, 3:5, 3:6, 3:7, 4:6, 4:7, 4:8 and four control conditions 1:1, 2:2, 3:3, 4:4 to monitor any laterality bias, i.e. going on the same side on every trial.

A correct response was scored if the subject chose the larger quantity first.

\section{b. Addition Numbers}

The experimenter placed three plates on the platform in front of the testing cage and put up an occluder to prevent the monkeys from watching the baiting procedure. Then she baited the three plates with different amounts of reward (same as in Relative Numbers) covered them with lids, and placed them in the middle of the platform. After the occluder was removed, the experimenter lifted the lids of the two outer plates simultaneously. After $\sim 5$ seconds had passed, the experimenter covered the two outer plates again and uncovered the plate in the middle. The monkeys were able to view the amount lying on the middle plate for $\sim 5$ seconds. Then the experimenter transferred the rewards from the middle plate to one of the side plates. During the transfer the subject could not see the content of the side plates. Then the experimenter removed the empty plate in the middle and the subject was allowed to choose between the two covered plates on the outer sides. Each subject received two trials for each of the following pairs (the order was randomized):

$1: 0+3: 0=4: 0 ; 6: 1+0: 2=6: 3,2: 1+2: 0=4: 1,4: 3+2: 0=6: 3,4: 0+0: 1=4: 1,2: 1+0: 2=2: 3,4: 3$ $+0: 2=4: 5$. Each of the combinations was presented with the resulting higher number being once on the left and once on the right side, resulting in 14 trials in total.

A correct response was scored if the subject chose the larger quantity first.

\section{Causality}

a. Noise

The experimenter placed two cups on the platform in front of the testing cage and put up an occluder to prevent the monkeys from watching the baiting procedure. Then she put a reward (peanut) in one of the two cups and closed them with a lid. After the occluder was removed one of two possible manipulations were performed:

Noise full: The experimenter shook the baited cup three times so that the food rattled inside and only lifted the empty cup without shaking it. Starting with the baited or empty cup was randomized.

Noise empty: The experimenter shook the empty cup (producing no sound) three times and then lifted the baited cup without shaking it. Starting with the baited or empty cup was randomized.

After the manipulations the subject was allowed to choose one cup. A correct response was scored if the subject chose the baited cup first.

\section{b. Shape}

The experimenter put up an occluder and placed two identical pieces of cardboard or cloth, respectively on the platform in front of the subjects test cage. Then the experimenter placed a 
reward (peanut) underneath one of the two identical objects causing a visible inclination or bump, respectively. After that the occluder was removed, and the subject was allowed to make one choice.

Board: The experimenter hid the reward underneath one of two cardboard boards $(15 \times 12 \mathrm{~cm})$. The reward caused a visually apparent inclination as it was placed on the food (the other board remained flat on the table).

Cloth: The experimenter hid the reward underneath one of the two pieces of cloth $(15 \times 10 \mathrm{~cm})$. The reward made a visible bump under this piece of cloth (the other cloth remained flat on the table).

A correct response was scored if subjects chose the baited board or cloth first.

\section{c. Tool Use}

A reward was placed on a table approximately $20 \mathrm{~cm}$ out of reach of the subject in front of their cage A wooden stick (20 cm in length) was provided for the subject. In this experiment no plastic panel was used. The animals could handle the stick through the wire mesh of their cage. To be successful the subject had to use the tool to retrieve the out of reach object or food within two to three minutes.

A correct response was scored if the subject was able to retrieve the reward.

\section{d. Tool Properties}

In these experiments a plastic panel with two oval openings on the left and right side was attached to the Plexiglas panel. The experimenter put up an occluder and placed two different tools on the platform in front of the testing cage. One tool was functional and could be used to retrieve a reward associated with it (e.g. lying on top of it), whereas the second tool was nonfunctional and could not be used to obtain the associated reward. In total five different objects were used:

Side: The experimenter put two identical pieces of cloth $(15 \mathrm{~cm} \times 10 \mathrm{~cm})$ on the platform behind an occluder and placed a reward on top of one cloth piece, whereas the other reward was placed directly next to the other cloth piece (i.e. making the second tool ineffective for retrieving the food). After the occluder was removed, the subject could only retrieve the reward by pulling the piece of cloth with the reward on top of it.

Bridge: The experimenter put two identical small plastic bridges over each of the far ends of the two identical cloth pieces behind an occluder. One reward was then placed on top of the bridge (making the tool ineffective in retrieving the food), the other reward was placed on the cloth underneath the bridge. After removing the occluder, the subject could only obtain the reward by pulling the cloth with the reward placed directly on it.

Ripped: The experimenter put up an occluder and placed a rectangular, intact cloth piece $(15 \mathrm{~cm}$ $x 10 \mathrm{~cm}$ ) on one side of the table, and two smaller cloth pieces $(9.5 \mathrm{~cm} \times 10 \mathrm{~cm}$ and $4.5 \mathrm{~cm} \times 10$ $\mathrm{cm}$ ) on the other side, arranging the small pieces of cloth in a way that there was a $1 \mathrm{~cm}$ gap between them. Then one reward was placed on top of the far end of the intact cloth, and the other reward was placed on the out of reach piece of the two disconnected pieces (making the tool ineffective to retrieve the reward). After removing the occluder, the subject could only acquire a reward by pulling the large, intact cloth piece.

Broken wool: The experimenter put up an occluder and placed two strings of wool on the platform, from which one was cut into two pieces. Like in the Ripped cloth condition both strings were arranged in a way that the gap was visible, but that both resulted in an equal 


\section{Chapter 2 - Monkeys Compare to Apes in the PCTB}

length. A peanut was tied to the far end of the wool strings out of the subject's reach. After removing the occluder, the reward could only be retrieved by pulling the intact piece of wool. Tray circle: The experimenter placed two small cardboard trays $(6 \mathrm{~cm} \times 6.5 \mathrm{~cm})$ on the platform behind an occluder. One tray had a hole cut out of it that formed a circle (3.5 $\mathrm{cm}$ in diameter) the other tray had a u-shaped hole, thus open to the end facing away from the monkey. A string was attached to both trays that could be used to pull the tray and the reward within reach. Then a reward was placed into the holes of each tray. In case of the u-shaped hole the tray surrounded the food but did not hold it. After removing the occluder, the subjects could only obtain the reward if they pulled the rope which was attached to the tray with the circle-shaped hole in it.

A correct response was scored if the subject first chose the functional tool by pulling it.

\section{Social Domain}

\section{Social Learning}

Before we conducted the social learning tasks, we established a baseline with 3 olive baboons and 3 long-tailed macaques. None of the six subjects solved the problem with the same means we demonstrated in the following three tasks (i.e. making it likely that any reproduction of the demonstrations described below are due to social influences). In the test conditions the subjects were given two minutes to solve the problem after the experimenter demonstrated the solution. To count as a correct response the subject had not only to obtain the reward but do so by using a highly similar procedure as the one demonstrated by the experimenter.

\section{a. Paper Tube}

The experimenter placed a reward inside a $30 \mathrm{~cm}$ long transparent plastic tub, which was coverd by two pieces of paper attached over both ends. Then she stood in front of the monkeys' cage and demonstrated how to open the tube: She held the tube in one hand and poked a hole into the paper with a finger. Then she ripped the paper further by twisting her finger in the tube. After that she tilted the tube and let the reward fall in her hand. After the demonstration she handed an identical tube to the subject.

\section{b. Banana Tube}

A slice of banana was placed in the center of a $30 \mathrm{~cm}$ long transparent Plexiglas tube. The banana was trapped in the tube and could only be retrieved by applying a specific force. The experimenter stood in front of the monkeys' cage and showed them how to retrieve the banana by banging one end of the Plexiglas tube on the floor. After the successful demonstration, she handed an identical tube with a banana inside to the subject.

\section{c. Stick Tube}

A $15 \mathrm{~cm}$ long opaque plastic tube with caps on each end was baited with a reward (peanut). One of the caps had a small hole in it but was tightly attached to the tube, whereas the other cap had no hole but could be removed. The experimenter stood in front of the monkeys' cage and demonstrated how to open the tube: She inserted a wooden stick through the cap with a hole, and pushed the stick through the hole which forced the cap on the other end to fall off. After the successful demonstration she handed an identical grey tube to the subject.

\section{Communication}

\section{a. Comprehension}


The experimenter placed two cups on the testing platform behind an occluder, one on the left and the other on the right side. Then she hid a reward under one of the cups. After removing the occluder she gave one of three social cues:

Look: The experimenter sat behind the platform and alternated her gaze between the subject and the baited cup three times while calling the subject's name. After these gaze alternations she continuously looked towards the cup until the subject chose.

Point: The experimenter sat behind the platform and continuously pointed to the baited cup with the extended index finger of her cross-lateral hand. At the beginning of the point, she alternated her gaze between the subject and the cup three times while calling the subject's name and then only stared in the baited cup's direction.

Marker: The experimenter held an iconic photo marker, which depicted the reward, in her hand and alternated her gaze three times between the photo and the subject while calling the subject's name. Then she placed the photo on top of the baited cup.

After the cue the subject was allowed to choose one cup. A correct response was scored if the subject chose the baited cup first.

\section{b. Production: Pointing Cups}

In the following task two experimenters were needed (E1 \& E2). Two cups served as hiding places for a food reward. These cups were placed on the outer part of the platform in front of the testing cage. The hiding places were spread apart (ca. $50 \mathrm{~cm}$ (macaques), ca. $70 \mathrm{~cm}$ (baboons)) and both equidistant from the subjects' starting point between the two hiding places. The second experimenter (E2) entered the testing area, placed a reward under one of the two cups while the subject was watching, and then left the area. Then E1 entered the testing area and centered the monkey by giving her a piece of food between the two cups through the middle hole in the Plexiglas panel. Then E1 stood equidistant to both cups and waited until the subject approached one cup and pointed towards it through a hole in the Plexiglas panel. A correct response was scored if the subject chose the correct cup within one minute.

\section{c. Production: Attentional State}

In the following task again two experimenters were needed (E1 \& E2). The second experimenter (E2) entered the testing area and placed a reward out of reach but in front of the subjects' cage on its right or left side. Then E2 left the area and E1 entered, but stood on the end of the room opposite of the reward and thus did not notice the reward on the floor. E1 stood and looked in four different ways:

Away: E1 turned around and looked away from the reward. When the monkey approached E1 from her front in order to see each other within 20 seconds (20s), E1 turned around and waited for the subject to direct her attention to the reward. If the subject went back to the reward's location and indicated the reward within 20s, E1 handed the reward to the subject.

Towards: E1 looked towards the reward. When the monkey approached the reward and directed E1 attention towards the reward within 20s, E1 handed it over to the subject.

Away Body-facing: Identical to Away, except that E1's body faced toward the reward and only the face was turned away. When the monkey approached E1 and directed her attention towards the reward within 20s, E1 handed it over to the subject. 
Towards Body-away: Identical to Towards, except that E1's body was turned away and only the face was directed towards the reward. When the monkey approached the reward and directed E1 attention towards the reward within 20s, E1 handed it over to the subject.

\section{Theory of Mind}

\section{a. Gaze Following}

The experimenter sat in front of the subject and gave it a piece of food to attract its attention. When the monkey sat and looked at the experimenter, she started the trial. The gaze cue was conducted in three different ways: (which were conducted on a different day within the test battery to minimize any kind of habituation):

Head + Eyes: The experimenter called the subject's name and showed them a piece of food. Then she hid the food in her hand, which remained in front of her body. She then looked up with both her head and eyes for $\sim 10$ s.

Back: The experimenter sat with her back facing the subject. She called the subject's name and showed them a piece of food. Then she hid the food in her hand, which remained in front of her body. She then looked up to the ceiling for $\sim 10$ s. Within the $\sim 10$ s she looked back over her shoulder at the subject three times to ensure that the subject was still paying attention. If the subject was not paying attention when the experimenter looked the second time, the trial was repeated.

Eyes: The experimenter called the subject's name and showed them a piece of food. Then she hid the food in her hand, which remained in front of her body. She then glanced up at the ceiling for $\sim 10$ s while her face was still facing the subject.

A correct response was obtained if the subject followed the gaze of the experimenter.

Straight: To control whether the subjects also gaze upwards without the experimenter looking up, we conducted this control condition. The experimenter called the subject's name land showed them a piece of food before hiding it in her hand, which remained in front of her body). However, instead of gazing upwards the experimenter looked straight forward at the subjects' chest.

\section{b. Intentions}

In these tasks two experimenters were needed (E1 \& E2). E1 put up an occluder and placed two cups on the platform in front of the testing cage. Then she hid a reward in one of the two cups. After removing the occluder, E2 manipulated the cups in one of two ways:

Trying: E2 reached for the baited cup and tried in vain to remove the lid while looking at the cup.

Reaching: A Plexiglas barrier blocked E2's access to the cups. Therefore, E2 unsuccessfully tried to reach the baited cup by extending the equilateral arm, looking at the correct cup. She continued to give this cue until the subject indicated a choice.

After each demonstration E1 approached the table after 3s and pushed the platform forward so that the subject was allowed to make a choice. To count as a correct response subjects had to choose the baited cup first. 
Table S1: Number of trials for each task and item

\begin{tabular}{|l|l|}
\hline Task/Item & Trials \\
\hline Spatial memory & $\mathbf{6}$ \\
\hline Object Permanence & $\mathbf{1 8}$ \\
Single displacement & 6 \\
Double adjacent displacement & 6 \\
Double non-adjacent displacement & 6 \\
\hline Rotation & $\mathbf{1 8}$ \\
$180^{\circ}$ middle & 6 \\
360 & 6 \\
180 ${ }^{\circ}$ side & 6 \\
\hline Transposition & $\mathbf{1 8}$ \\
Single transposition & 6 \\
Double unbaited transposition & 6 \\
Double baited transposition & 6 \\
\hline Relative Numbers & $\mathbf{1 6}$ \\
\hline Addition Numbers & $\mathbf{1 4}$ \\
\hline Noise & $\mathbf{1 2}$ \\
Noise full & 6 \\
Noise empty & 6 \\
\hline Shape & $\mathbf{1 2}$ \\
Board & 6 \\
Cloth & 6 \\
\hline Tool use & $\mathbf{1}$ \\
\hline Tool properties & $\mathbf{3 0}$ \\
Side & 6 \\
Bridge & 6 \\
Ripped & 6 \\
Broken wool & 6 \\
Tray circle & 6 \\
\hline & \\
\hline
\end{tabular}

\begin{tabular}{|l|l|}
\hline Task/Item & Trials \\
\hline Social learning & $\mathbf{3}$ \\
Paper tube & 1 \\
Banana tube & 1 \\
& 1 \\
\hline Comprehension & $\mathbf{1 8}$ \\
Look & 6 \\
Point & 6 \\
Marker & 6 \\
\hline Pointing Cups & $\mathbf{8}$ \\
\hline Attentional State & $\mathbf{4}$ \\
Away & 1 \\
Towards & 1 \\
Away Body-facing & 1 \\
Towards Body-away & 1 \\
\hline Gaze following & $\mathbf{9}$ \\
Head \& Eyes & 3 \\
Back & 3 \\
Eyes & 3 \\
\hline Intentions & $\mathbf{1 2}$ \\
Trying & 6 \\
Reaching & 6 \\
\hline
\end{tabular}




\section{File S2}

\section{Assessment of Temperament, Inhibitory Control and Rank Temperament test}

To assess differences in temperament between the monkeys we also used the same methods as Herrmann et al. (2007). To assess differences in the temperament of the subjects we tested their reaction to novel objects, persons and foods. When appropriate we also used the same wording to describe the tasks as Herrmann et al. The test situation varied concerning 1) the nature of the different items shown (humans, objects or food pieces), 2) whether the items were presented alone or in combination (e.g. human moving a novel object) and 3) whether the objects were moved or not or could be touched during their presentation (e.g. item was moved from left to right by the experimenter, etc.). We measured whether the subjects approached the new items, how fast they did that and whether they tried to touch the presented objects.

\section{Procedure and Design}

Similar to the temperament tests done by Herrmann et al. (2007) each monkey participated in 29 different items which could be grouped into four categories: human, object, food/reward and nonhuman (see Table S2). For the presentation of each item the unfamiliar experimenter (E1) sat in front of the monkeys' cage (excluding the familiar human and nonhuman condition). A second, familiar experimenter (E2) made sure that the subject was at a designated starting point by offering food. When the monkey was in the correct place E1 presented the different stimuli for 30 seconds each. The subjects received one session per day and the objects were presented in the same order across all subjects. On the first day (Visible) the subjects were only able to view the experimenter sitting behind the table and the different items were placed on the table. On this day we also conducted two additional non-social trials in which the monkeys could either view the table alone or when a bright red spot was placed on the table top before E1 left the area. On the second day (Movement) the different items were moved from left to right during the 30 s of presentation by the experimenter. On the third day (Touch) the items were put close to the monkeys' cage so that the subjects were able to touch them.

During all of the experiments a camera filmed a predetermined 'visible area' (including the items and about $2 \mathrm{sqm}$ of the cage near the items). From the videos we then recorded three measures: latency (time to come into the proximity of the object), duration (time spent near the object), and proximity (how close the monkeys approach the object).

Table S2: Items and Methods used in the Temperament Test.

\begin{tabular}{|l|l|l|l|}
\hline Visible & Category & Item & Description \\
\cline { 2 - 4 } & Human & $\begin{array}{l}\text { - Familiar } \\
\text { - Non-Familiar (E1) }\end{array}$ & The Person sat behind the table facing the mesh. \\
\cline { 2 - 4 } & $\begin{array}{l}\text { - Orange ashtray } \\
\text { - Plastic Beetle } \\
\text { - Police car }\end{array}$ & $\begin{array}{l}\text { E1 sat behind the table, hands on her lap with the } \\
\text { object placed in the middle of the table. } \\
\text { In the police car condition E1 held the remote } \\
\text { control and pressed the horn button ten times. }\end{array}$ \\
\cline { 2 - 4 } & $\begin{array}{l}\text { Food } \\
\text { - - Fruit piece } \\
\text { - 3 Peanuts } \\
\text { - Lemon }\end{array}$ & $\begin{array}{l}\text { E1 sat behind the table, hands on her lap with the } \\
\text { food placed in the middle of the table. }\end{array}$ \\
\cline { 2 - 4 } & Non- & - Red spot & E1 placed a red spot in the middle of the table and \\
\hline
\end{tabular}




\begin{tabular}{|c|c|c|c|}
\hline & Human & - Nothing & $\begin{array}{l}\text { left. } \\
\text { Nothing is on the table and E1 is out of sight. }\end{array}$ \\
\hline \multirow[t]{3}{*}{ Movement } & Human & $\begin{array}{l}\text { - Hand } \\
\text { - Body }\end{array}$ & $\begin{array}{l}\text { E1 sat behind the table, moved her right hand from } \\
\text { the left side to the right side. } \\
\text { In the body condition E1 nodded up and down. }\end{array}$ \\
\hline & Object & $\begin{array}{l}\text { - Orange Ashtray } \\
\text { - Plastic Beetle } \\
\text { - Police car }\end{array}$ & $\begin{array}{l}\text { E1 sat behind the table, moved the object from the } \\
\text { left side to right side and back on the table. } \\
\text { In the police car condition E1 let the car drive to the } \\
\text { on the table. }\end{array}$ \\
\hline & Food & $\begin{array}{l}\text { - Undesirable food } \\
\text { - Fruit piece } \\
\text { - } 3 \text { Peanuts } \\
\text { - Lemon }\end{array}$ & $\begin{array}{l}\text { E1 sat behind the table, moved the food from the } \\
\text { left } \\
\text { side to right side and back on the table. }\end{array}$ \\
\hline \multirow[t]{3}{*}{ Touch } & Human & - Hand & $\begin{array}{l}\text { E1 sat behind the table, put her right hand as a fist } \\
\text { on the table. }\end{array}$ \\
\hline & Object & $\begin{array}{l}\text { - Orange Ashtray } \\
\text { - Plastic Beetle } \\
\text { - Police car } \\
\text { - Box }\end{array}$ & $\begin{array}{l}\text { E1 sat behind the table, hands on her lap with the } \\
\text { object placed on the table within reach of the } \\
\text { subject. }\end{array}$ \\
\hline & Food & $\begin{array}{l}\text { - Undesirable food } \\
\text { - Fruit piece } \\
\text { - } 3 \text { Peanuts } \\
\text { - Lemon }\end{array}$ & $\begin{array}{l}\text { E1 sat behind the table, hands on her lap with the } \\
\text { food placed on the table within reach of the subject. }\end{array}$ \\
\hline
\end{tabular}

\section{Analyses and Results}

To analyse the differences between baboons and macaques in the three measures (i.e. latency, duration or proximity) we used the same methods as for the performance in the different PCTB tasks (MANOVA and ANCOVA). To compare the results of these temperament tests to the performance in the PCTB we first determined the most indicative temperament variable. Therefore, we did a variance component analysis to examine which factors (i.e. individual, item, or procedure) best explained the variation in the three measurements and compared this to the performance in the PCTB (Pearson correlations).

The variance component analyses revealed that the measure "Duration" accounted for the largest differences between individuals. With this measure we then conducted Pearson correlation analyses with the performance in the physical and social domain, which were all not significant.

Concerning species there was a significant difference between the baboons and macaques (MANOVA with species and sex as between-subject factor and scores in the three measurements as dependent variables; $\left.F(3,11)=28.72, p<.001, \eta^{2}=.887\right)$. Post hoc tests showed that the baboons spent more time next to new objects than the macaques $(p<.001)$ and approached new stimuli faster $(p=.002)$. There was also a significant effect of sex (Wilk's Lambda, $F(3,11)=5.89, p<.012, \eta^{2}=.617$ ) and interaction between species and sex (Wilk's Lambda, $\left.F(3,11)=5.05, p<.019, \eta^{2}=.579\right)$ with male baboons staying significantly longer near new items than female baboons (Posthoc-test, $p=.011$ ) and longer than male and female macaques (Posthoc-tests, $p<.001$ ). Furthermore, female macaques took significantly longer to approach a new item than female (Posthoc-test, $p=.023$ ) and male baboons (Posthoc-test, $p=$ .002). When controlling for age these differences still remain significant (ANCOVA with species 
and sex as between-subject factor, age as covariate and scores in the three measures as dependent variables, all $p<.034$ ).

\section{Inhibitory control test}

To examine whether the amount of inhibitory control correlates with the monkeys' performance in the PCTB, we conducted the same inhibitory control tasks as Herrmann et al. (2007). We conducted three additional trials within the spatial memory tasks. Rewards were placed under two out of three cups while the subject was watching. For these trials, however, only the two outer cups were baited, while the middle cup was left empty. If the subject first chose one of the outer cups it was allowed to make a second choice. If, however, it chose the middle cup first, no further choices were possible. A correct response was scored when the monkey chose the two outer cups in succession while skipping the middle cup.

We found no significant difference between the performance of the baboons and macaques (Mann-Whitney U-Test, $z=0, p=1$ ).

\section{Rank}

To examine whether rank has an influence on the subjects' performance we completed focal observations for each individual of the long-tailed macaques, which allowed us to classify them as high, middle or low ranking (see Table S3). Focal observations lasted 120 min (6 20 min) for each animal. Rank was calculated by subtracting the number of events in which aggression was received from the number of events aggression was given, corrected for the total number of aggressive events observed per animal. The resulting score determined whether a subject was considered as high, middle or low ranking. The rank of the baboons was estimated by personal observations (VS) over 2.5 years.

Table S3: Results of the focal observations done on the monkeys and their classification as high, middle or low ranking.

\begin{tabular}{|c|c|c|}
\hline $\begin{array}{c}\text { Subject } \\
\text { Macaque }\end{array}$ & $\begin{array}{l}\text { (Aggression given- } \\
\text { Aggression received)/N of } \\
\text { Aggression }\end{array}$ & Rank \\
\hline $\mathrm{Su}$ & 0.92 & $\mathrm{~h}$ \\
\hline $\mathrm{Ma}$ & 0.76 & $\mathrm{~h}$ \\
\hline $\mathrm{IS}$ & 0.70 & $\mathrm{~h}$ \\
\hline $\mathrm{Po}$ & 0.26 & $\mathrm{~m}$ \\
\hline $\mathrm{Pa}$ & 0.11 & $\mathrm{~m}$ \\
\hline $\mathrm{Sa}$ & 0.11 & $\mathrm{~m}$ \\
\hline $\mathrm{Pi}$ & 0.00 & $\mathrm{~m}$ \\
\hline Se & -0.08 & $\mathrm{~m}$ \\
\hline Le & -0.11 & $\mathrm{~m}$ \\
\hline So & -0.54 & $\mathrm{I}$ \\
\hline Sam & -0.57 & $\mathrm{I}$ \\
\hline Li & -0.78 & $\mathrm{I}$ \\
\hline Sun & -1.00 & $\mathrm{I}$ \\
\hline
\end{tabular}

\begin{tabular}{|c|c|}
\hline $\begin{array}{c}\text { Subject } \\
\text { Baboon }\end{array}$ & Rank \\
\hline $\mathrm{Pk}$ & $\mathrm{h}$ \\
\hline $\mathrm{Ms}$ & $\mathrm{h}$ \\
\hline $\mathrm{Bh}$ & $\mathrm{m}$ \\
\hline $\mathrm{Jg}$ & $\mathrm{m}$ \\
\hline $\mathrm{Tg}$ & $\mathrm{l}$ \\
\hline
\end{tabular}

$$
\begin{array}{ll}
> & >0.5=\text { High } \\
> & -0.5-0.5=\text { Middle } \\
> & <-0.5=\text { Low }
\end{array}
$$


CHAPTER 3

\title{
Representational Format Determines Numerical Competence in Monkeys
}

\author{
Vanessa Schmitt ${ }^{1,2}$ \& Julia Fischer ${ }^{1,2}$ \\ ${ }^{1}$ Cognitive Ethology Lab, German Primate Center, Göttingen, Germany \\ ${ }^{2}$ Courant Research Centre Evolution of Social Behaviour, University of Göttingen, \\ Germany
}

Nature Communications 2, 257 (2011)

DOI: $10.1038 /$ ncomms1262

\section{Author Contributions}

VS and JF designed the study; VS collected the data; JF analysed the data; both authors contributed substantively to the writing and editing of the final draft 


\title{
Chapter 3-Quantity Discrimination
}

\begin{abstract}
A range of animal species possess an evolutionarily ancient system for representing number, which provides the foundation for simple arithmetical operations such as addition and numerical comparisons. Surprisingly, nonhuman primates tested in ecologically, highly valid quantity discrimination tasks using edible items often show a relatively low performance, suggesting that stimulus salience interferes with rational decision making. Here we show that quantity discrimination was indeed significantly enhanced when monkeys were tested with inedible items compared with food items ( 84 versus $69 \%$ correct). More importantly, when monkeys were tested with food, but rewarded with other food items, the accuracy was equally high (86\%). The results indicate that the internal representation of the stimuli, not their physical quality, determined performance. Reward replacement apparently facilitated representation of the food items as signifiers for other foods, which in turn supported a higher acuity in decision making.
\end{abstract}




\section{Introduction}

The ability to judge quantities is of great relevance in a variety of ecological contexts, such as predation, foraging, and breeding (Dehaene 1997). Previous research conducted in the laboratory and in the field has provided compelling evidence that numerical abilities are not exclusively human (for reviews see Feigenson et al. 2004; Nieder 2005). Some basic arithmetical skills have been shown in dogs, cats, chicks and even mosquitofish (Gambusia holbrooki) (Agrillo et al. 2007; Ward \& Smuts 2007; Rugani et al. 2008; Pisa \& Agrillo 2009), suggesting that a broad array of species possesses an evolutionarily ancient system for representing numbers.

On the assumption that food quantity discrimination is ecologically highly valid, numerical skills of animals have frequently been examined through testing whether they are able to select the larger of two food quantities (relative numerousness judgment) (Beran et al. 2005; Anderson et al. 2007; Beran et al. 2008; Evans et al. 2009). A further advantage of using food quantity discrimination is that it avoids complicated or time-consuming training procedures, which must be employed when symbols are used (Olthof et al. 1997; Addessi et al. 2008a).

Somewhat counter-intuitively, however, studies using simple food quantity discrimination paradigms often report performance at relatively low levels. Western lowland gorillas (Gorilla gorilla gorilla), for instance, performed at chance level in a food quantity discrimination task and only learned to select the larger of two food quantities after additional training (Anderson et al. 2005). Similarly, chimpanzees and orang-utans performed only at about $65 \%$ correct in a relatively simple food quantity discrimination task (Herrmann et al. 2007). But why is this the case? From reversed reward paradigms it is known that the salience of the choice stimulus is a crucial factor in experiments. In this paradigm, animals have to point to the smaller quantity to obtain the larger one. Chimpanzees fail at this task, as they appear to be unable to inhibit reaching towards the larger food amount. In contrast, when symbols (Arabic numerals) were used, they did significantly better (Boysen \& Berntson 1995).

To gain a better understanding of the factors that support accurate decision making, we tested Old World monkeys in a series of quantity discrimination tasks. We conducted twochoice experiments with olive baboons (Papio anubis) and long-tailed macaques (Macaca fascicularis) in which they had to discriminate between arrays of edible and inedible items (pebbles). We predicted that the monkeys would perform better when tested with inedible, that is, less salient items. There are two possible explanations. For one, the highly salient food items might impair impulse inhibition. Alternatively, the monkeys might have difficulties to simultaneously maintain two mental representations of the food items, first as choice stimulus and second as food reward (DeLoache 2000). To distinguish between these two possible 
explanations, we introduced a third condition in which the monkeys were required to discriminate between food items, but under a different reward contingency scheme: in this experiment, the subjects were not rewarded with the food items they had pointed at, but with other food items of the same kind as the choice stimuli hidden underneath the plates presenting the food.

Our results revealed that the reward contingency is more important than stimulus salience, as subjects performed equally well when tested with inedible items and when food items were replaced. These findings suggest that the mental representation of what choice stimuli stand for is more important for controlling choice behaviour than physical appearance of the stimuli.

\section{Results}

Procedure. We tested 16 Old world monkeys (olive baboons and long-tailed macaques) housed at the German Primate Center in a two-choice paradigm. Animals were presented simultaneously with two different amounts (1-8 items) of edible (raisins or peanuts) or inedible items (pebbles). Quantities differed in magnitude from 1 to 4 (Table 1). After the subjects made their choice by pointing at the desired quantity, they were rewarded either with the food items they had pointed at or with an amount of food equivalent to the amount chosen. To accustom the monkeys to the respective choice paradigm, they passed a short familiarization phase before the actual test phase of each condition began. There was no significant difference in performance between the two species across conditions (generalized linear mixed model (GLMM) analysis with Monte-Carlo-Markov chain (MCMC) procedure: $N=16, P=0.25$, Table 2); therefore, results are presented for the pooled data set.

Table 1. Absolute difference and ratios of the quantities used in the experiments.

\begin{tabular}{cccccc} 
Difference & $\mathbf{0}$ & \multicolumn{1}{c}{$\mathbf{1}$} & \multicolumn{1}{c}{$\mathbf{2}$} & \multicolumn{1}{c}{$\mathbf{3}$} & \multicolumn{1}{c}{$\mathbf{4}$} \\
\hline & $1: 1$ & $2: 1(2.0)$ & $3: 1(3.0)$ & $4: 1(4.0)$ & $5: 1(5.0)$ \\
Combinations & $2: 2$ & $3: 2(1.5)$ & $4: 2(2.0)$ & $5: 2(2.5)$ & $6: 2(3.0)$ \\
& $3: 3$ & $4: 3(1.33)$ & $5: 3(1.67)$ & $6: 3(2.0)$ & $7: 3(2.33)$ \\
& $4: 4$ & $5: 4(1.25)$ & $6: 4(1.5)$ & $7: 4(1.75)$ & $8: 4(2.0)$
\end{tabular}

Difference refers to the absolute difference between the two amounts of items used in the test. Equal amounts (difference $=0$ ) served as control condition. The numerical ratios (the larger divided by the smaller quantity) are given in parentheses. 
Test conditions. In the ecologically most valid 'Food' condition, food items were used as choice stimuli and as rewards; that is, the food items selected by the monkey were fed to her (Supplementary Movie 1). In this condition, the monkeys chose the larger amount above chance but at relatively low levels ( $68.8 \%$ of the choices; Fig. 1 ). When small black pebbles served as choice stimuli, and the animals were rewarded with the equivalent amount of food items (Pebble condition), subjects chose the larger amount of items significantly more frequently (84.4\%; Fig. 1, Table 2; $N=16$; post hoc test between these two conditions: $P<0.001$ ).

In the 'Food replaced' condition, the subjects were rewarded with other food items hidden underneath the plates presenting the food. In this condition, the choice stimulus was highly salient, while choice stimulus and reward were separate entities, that is, the reward contingency was the same as in the non-food condition. If the performance of the monkeys is determined by the quality of the stimulus (being edible or not), they should obtain similar poor results in the Food and 'Food replaced' conditions. In contrast, if the reward contingency is decisive, they should do well both when tested with inedible items and when rewarded with other food items. In this condition, the subjects performed at a similar level as in the 'Pebbles' condition (see Fig. 1, post hoc test: $P=0.59$ ) and significantly better than in the initial 'Food' condition (Supplementary Movie 2). They chose the larger amount in $85.6 \%$ of all trials (post hoc test: $P<0.001)$.

The performance of the animals in all three test conditions was influenced by the absolute magnitude of the difference between the two quantities as well as the ratio between the two quantities (GLMM analysis with MCMC procedure, Fig.2 and Table 2).

Table 2. Effects of the different predictor variables on performance.

\begin{tabular}{lcccc} 
Predictor & Estimate & s.e. & $\boldsymbol{t}$ & $\mathbf{P}_{\text {MCMC }}$ \\
\hline (Intercept) & 0.108 & 0.173 & 0.624 & 0.5554 \\
Species & -0.042 & 0.034 & -1.237 & 0.2452 \\
Condition & 0.019 & 0.003 & 7.402 & 0.0001 \\
Difference & 0.042 & 0.011 & 3.740 & 0.0001 \\
Ratio (In) & 0.101 & 0.033 & 3.027 & 0.0024
\end{tabular}

Condition refers to the three experimental conditions Food, Pebbles and Food replaced; difference refers to the absolute difference between the two amounts presented and ratio (In) to the In-transformed ratios between the two quantities. Parameter, s.e. (standard error) of the Estimate and $t$-value (test statistic) were obtained from a GLMM analysis. A Monte-Carlo-Markov-Chain procedure was used to approximate the significance levels of the parameter estimates $\left(P_{\mathrm{MCMC}}\right)$. 


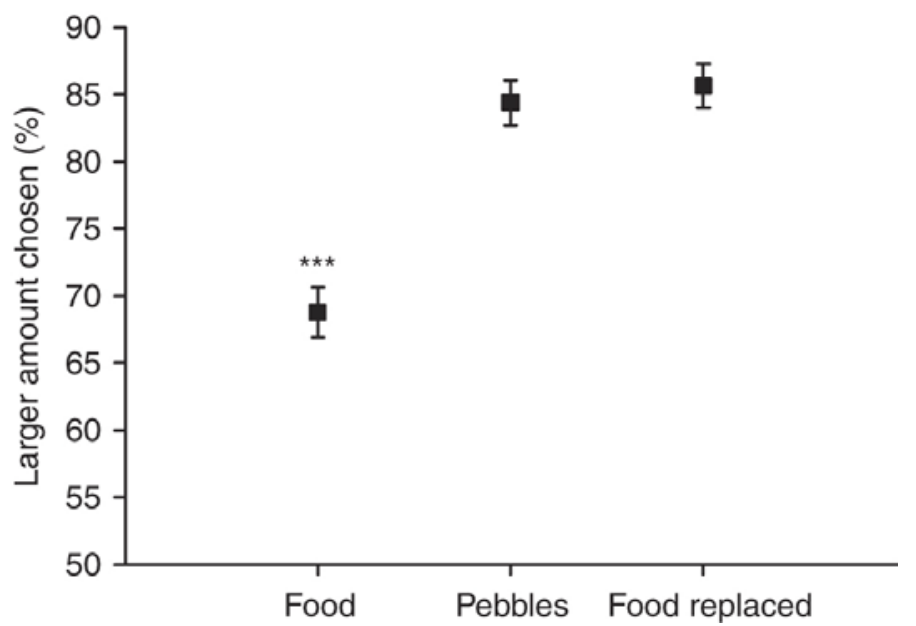

Figure 1 | Percent of trials in which the larger quantity was chosen in the different test conditions (means and standard error of means). Performance in the 'Food' condition was significantly worse than in the other two conditions (GLMM, $N=16$ subjects; $P<0.001$ ).

Control Conditions. To test the hypothesis that the poor performance in the Food condition was due to the changing appearance of the choice stimuli while the food items were given to the monkeys, leading to a decrease in associative strength of these stimuli, we added two types of control conditions. Varying the appearance of the stimuli by either removing all food stuffs before giving them to the monkeys, or by removing a pebble each time one food items was given to the subject did not change the pattern: monkeys were still significantly better when discriminating between different amounts of pebbles ( $86 \%$ correct) compared to food items (75\% correct; $P<0.001)$.

To test whether unintentional cueing by the experimenter might have affected the monkeys' performance ('Clever Hans effect'), we ran an additional control. In these experiments, we used boxes with a lid that opened to one side and small drawers to deposit the corresponding amount of food pieces. The boxes were baited by a second experimenter so that the first experimenter did not know how many pebbles were in each box. She then presented the boxes to the subjects and opened the lids so that the monkeys could see the content of the boxes while the experimenter could not. After choosing, the monkeys were rewarded with the food items in the corresponding box. There were no significant differences in performance in relation to whether the monkeys were tested in the regular 'Pebbles' condition or in the 'Experimenter blind' condition (mean \pm s.e.m. performance in the regular condition $0.81 \pm 0.03$; and $0.81 \pm 0.02$ in the Experimenter blind condition; $T=10.5, N=8, P=1$; exact Wilcoxon signed-ranks test). 
a

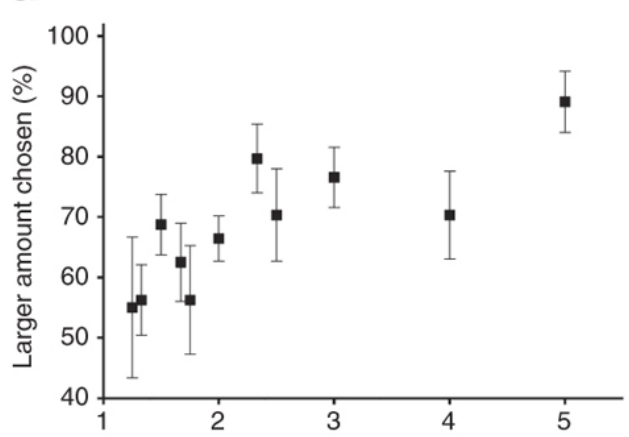

b

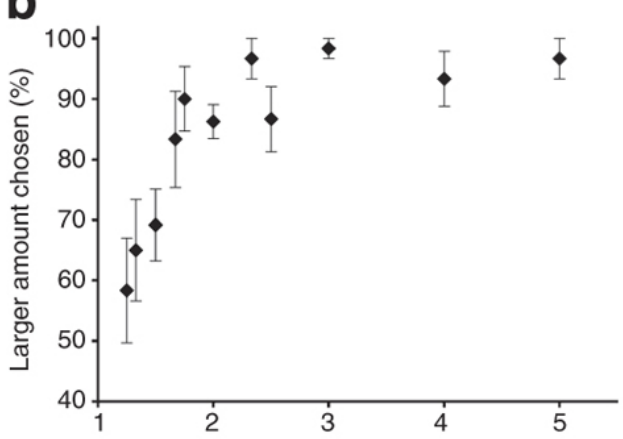

C

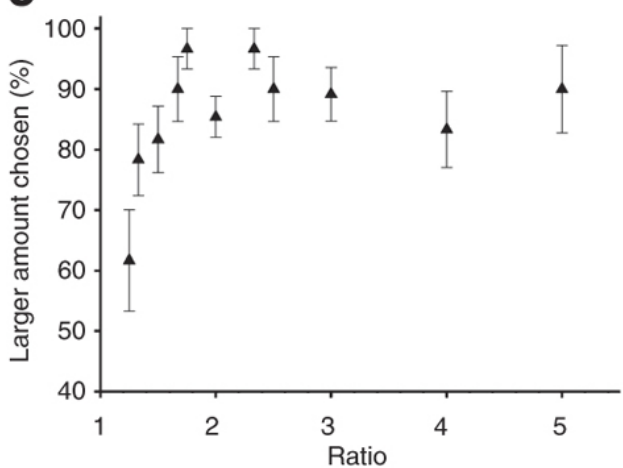

d

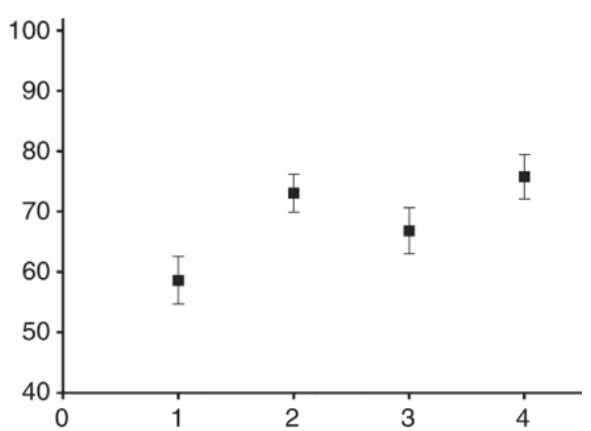

e

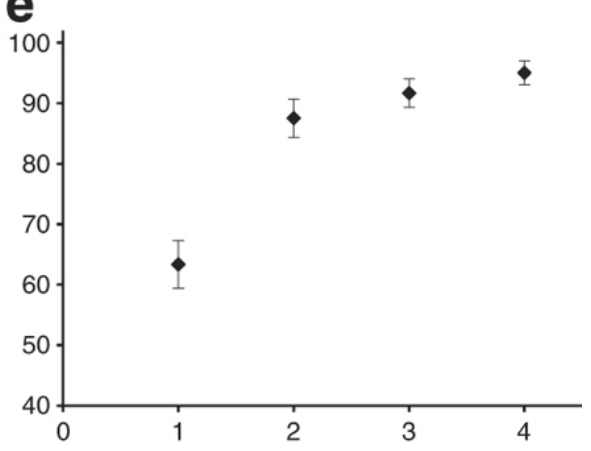

f

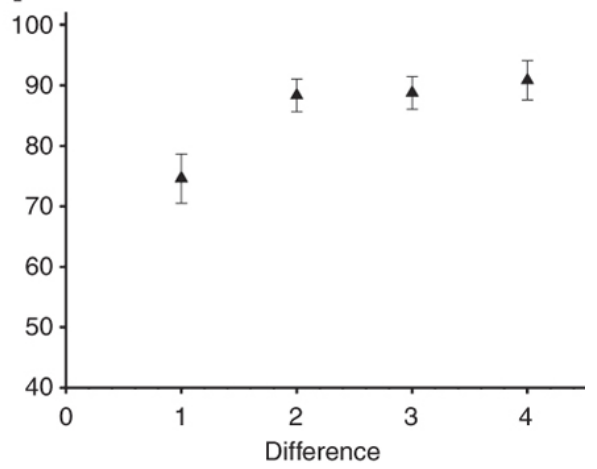

Figure 2 | Effects of relative and absolute difference between choice stimuli on performance. Percent of trials in which the larger amount was chosen (means and standard error of means) in relation to the ratio between the quantities presented for the three different conditions (a) Food, (b) Pebbles, (c) Food replaced; and in relation to the absolute difference between quantities (d) Food, (e) Pebbles, (f) Food replaced. There was a combined effect of relative and absolute difference on performance (GLMM, $N=16$ subjects, Effect of absolute difference $P=$ 0.0001 , Effect of ratio $P=0.0024$ ).

\section{Discussion}

In the ecologically most valid 'Food' condition the monkeys chose the larger amount above chance but at relatively low levels. In contrast, subjects chose the larger amount of items significantly more frequently in the 'Pebbles' condition. Thus, the monkeys' poor performance in the 'Food' condition was not due to an inability to discriminate between the different 


\section{Chapter 3 - Quantity Discrimination}

quantities. These results are compatible with the notion that highly salient stimuli impair impulse inhibition. Likewise, human children performed significantly better when symbolic representations substituted for real candies in the reversed-reward paradigm (Carlson et al. 2005), similar to the results obtained with chimpanzees (Boysen \& Berntson 1995).

Strikingly, in the critical 'Food replaced' condition, the subjects performed at a similar level as in the 'Pebbles' condition and significantly better than in the initial 'Food' condition. This finding refutes the assumption that a lack of impulse inhibition is the sole explanation for the poor performance in the 'Food' condition (see also Shifferman 2009 for alternative explanations of the reversed-reward paradigm). Instead, the internal representation of the choice stimuli, not their physical quality, seems to be crucial for the improved performance. In particular, it appears that the monkeys fail to master the dual representation of the stimuli as choice stimulus and as food reward.

"Dual representation", that is, the mental representation of an object as well as the representation of the relation between an object and what it stands for, is seen as a foundation of abstract reasoning and symbolic understanding (DeLoache 2000). Research on children has shown that increasing the salience, that is, attractiveness of an object, impairs dual representation (DeLoache 2000). Our results suggest that in the 'Food' condition, our subjects failed at this dual representation, in the sense that they were unable to simultaneously maintain both representations of the items as food and as choice stimuli. In the 'Food replaced' condition, in contrast, the representation as food was diminished and that as signifiers for different quantities enhanced. This in turn supported the increase in accuracy.

Representing food items as choice stimuli can be seen as a form of representational redescription (RR). RR is posited as a process by which implicit information in the mind becomes explicit knowledge to the mind by recoding information from one representational format to another (Karmiloff-Smith 1992). Thus, the stimuli become available to explicit reasoning and decision making. Clearly though, this elementary form of RR needs to be distinguished from relational RR as described by Penn and colleages (Penn et al. 2008). Relational RR involves structurally systematic, rule-governed relational redescriptions, and, has been suggested to be a distinguishing feature of humans.

Overall, the performance of our subjects was comparable to those of other monkey species (Brannon \& Terrace 1998; Beran 2007) and great apes (Herrmann et al. 2007). Their accuracy was influenced by the absolute magnitude of the difference as well as the ratio between the two quantities. Two different mechanisms have been invoked to account for numerousness judgments, the analogue magnitude and the object-file model (for review see Hauser \& Spelke 
2004). The analogue magnitude model estimates large numerical magnitudes and is characterized through: (1) less accurate discrimination as the size of quantities increases and (2) as the ratio between the larger divided by the smaller magnitude decreases (Weber's law) (Dehaene et al. 1998). The object-file model predicts a decline in discrimination ability when more than four items are to be judged. It operates by keeping track of individual objects and therefore serves for the representation of small exact numerosities. A range of studies found support for the analogue magnitude model (Call 2000; Nieder \& Miller 2004), whereas others favoured the object-file model (Hauser et al. 2000). Our results are in line with the assumptions of the analogue magnitude model, because performance was poor when the ratio approached 1. In contrast, they are not compatible with the object-file model because subjects were still very good at discriminating between large quantities, for example, 7 and 3 items, and showed poor performance when the difference was small.

Our results have two main implications. First, we demonstrate that quantity discrimination paradigms using food may underestimate the true competence of a species (Spelke \& Hespos 2001). Second, we provide further insight into the conditions that favour rational decision making, specifically the effects of reducing the appetitive value of the choice stimulus. Taken together, our findings mirror those made with children (DeLoache 2000) and suggest that the basic cognitive operations that facilitate abstract reasoning have deep evolutionary roots (Addessi et al. 2008b; Diester \& Nieder 2010).

\section{Methods}

Subjects. Six olive baboons (Papio anubis) - four males and two females aged 3-9 years-living in a group of 11 animals and 10 long-tailed macaques (Macaca fascicularis)-five males and five females aged 1-7 years-living in a group of 32 animals were tested. The animals were housed at the German Primate Center in Göttingen and had access to indoor (baboons: 17 sqm, macaques: $40 \mathrm{sqm}$ ) and outdoor areas (baboons: 81sqm, macaques: $141 \mathrm{sqm}$ ). They were individually tested in their familiar indoor cages. Water was always available ad libitum and subjects were not food deprived for testing.

Materials. Two round white plastic plates (height $0.01 \mathrm{~m}$, diameter $0.08 \mathrm{~m}$ ) were baited with different amounts of food items, that is, raisins or pieces of peanuts (one piece corresponds to half a peanut), depending on food preferences, or little black pebbles ( $0.01 \mathrm{~m}$ in diameter) and put on a sliding table in front of the subject. The sliding board consisted of grey polyvinylchloride (length $0.8 \mathrm{~m}$, width $0.27 \mathrm{~m}$, height $0.01 \mathrm{~m}$ ) and was attached to a fixed polyvinylchloride table (length $0.8 \mathrm{~m}$, width $0.38 \mathrm{~m}$, height $0.01 \mathrm{~m}$ ) by two drawer rails so that the sliding table could be moved horizontally. The sliding table was attached with an iron mount 


\section{Chapter 3 - Quantity Discrimination}

in front of a plastic panel (height $0.7 \mathrm{~m}$, width $0.8 \mathrm{~m}$ ). The plates were placed on the right and left side of the sliding table. Two holes (diameter $0.01 \mathrm{~m}$, distance $0.3 \mathrm{~m}$ ) in the plastic panel allowed the subjects to point with their fingers at the cups. It was possible to set up an occluder of grey plastic (length $0.8 \mathrm{~m}$, height $0.3 \mathrm{~m}$, thickness $0.03 \mathrm{~m}$ ) in front of the panel so that the subject was not able to watch the baiting procedure. All sessions were videotaped with a digital video camera (Sony DCR-HC90E).

Procedure. Before each test condition, the subjects went through a familiarization phase to accustom them to the choice paradigm used in the following test condition. The procedure was the same in the familiarization and the corresponding test condition. The experimenter placed the two plates in the middle of the sliding table and baited every plate with the designated number of pieces (food or pebbles) behind an occluder, trying to avoid consistent arrangements of the choice stimuli. The occluder was removed and the experimenter waited until the animal paid attention (usually, they were already sitting in front of the table). Then the two plates were simultaneously moved in front of the two holes. After that the sliding table was pushed against the Plexiglas panel and the subject was allowed to choose. To avoid cueing the subject, the experimenter looked at the middle of the Plexiglas panel during the whole procedure (see also controls below). A choice was coded when the subject pointed with one finger to one of the locations through a hole in the screen. In the familiarization phase, the subjects were offered only two types of pairwise combinations (that is, 7 versus 1 and 8 versus 2) with 10-16 trials per session, one session per day. After reaching $80 \%$ correct responses within a session (always accomplished in the first or second session), the corresponding test session with different quantity combinations began. After the completion of each test condition, the subjects went through the new familiarization phase to introduce them to the paradigm of the next condition.

In the test phase, we used the following conditions: Food: The experimenter put up the occluder in front of the plates and baited them with the designated quantity of food items (raisins or peanuts). Next, the occluder was removed, the plates were moved in front of the two holes and the sliding table was pushed against the Plexiglas panel. After the subject had made its choice, it received all the food items on the plate it had pointed at. Pebbles: The experimenter put up the occluder and placed the same number of food items (raisins or peanuts) underneath the plates as pebbles were placed onto the plates. Then the occluder was removed, the plates were moved in front of the holes, the table was pushed forward and the subjects could choose. The monkeys then received all food items under the plate they had pointed at. 'Food replaced' condition: The experimenter put up the occluder and placed the same number of food items (raisins or peanuts) underneath the plates as food items were placed onto the plates. For all baboons, raisins were put onto the plates and the same number of pieces of peanuts was put underneath. We can exclude that they may prefer peanuts to raisins and perform better because of this simple explanation, as some of the baboons did not want to take the peanuts near the end of the sessions, so we used raisins instead. In these trials, 
raisins were placed on top of the plates as well as underneath. The baboons performed equally in these trials and in the rest of these sessions. However, to exclude any inferences from using different food kinds as choice stimuli and reward, we always used the same kind of food as choice stimuli and reward for the macaques, thus peanuts and peanuts or raisins and raisins depending on the food preferences of each subject. After baiting, the occluder was removed, the plates were moved in front of the holes, the table was pushed forward and the subject could choose. The monkeys then received all peanuts or raisins, respectively, under the plate they pointed at. The subject's responses were initially coded live by the experimenter. To test for observer reliability $30 \%$ of all trials $(N=740)$ were independently scored by a second coder. The inter-observer reliability was excellent (Cohen's k: 0.98).

Design. Initially, we started the study with the baboons. Every subject received four sessions per condition (one session per day; except the baboon $\mathrm{BH}$ that received only two sessions in the 'Food replaced' condition and the baboon MC that participated only in the 'Food' condition because of motivational problems). One session consisted of 20 trials resulting in 80 trials per condition per animal, thus a total of 240 trials per animal (but only 200 for $\mathrm{BH}$, and 80 for MC). Each session included five numeric differences (four experimental and one control difference), ranging from 0 to 4 . Within each numeric difference, there were four trials with different quantities of items used (Table 1). The sequence of the trials was balanced and the position of the larger quantity was counterbalanced across sessions. The baboons received the conditions in the following order: Food, Pebbles and 'Food replaced' condition. To exclude a learning effect across all conditions we repeated the initial 'Food' condition at the end. The baboons performed equally as in the first condition (70\% correct), thus learning the different quantity combinations could not account for the differences in their performance in the other conditions.

To test the consistency of the results found for the baboons, we repeated the test with longtailed macaques. Every subject received two sessions (one session per day) in each of the three test conditions. One session consisted of 20 trials resulting in 40 trials per condition per animal, thus a total of 120 trials per animal. The design of the sessions was the same as for the baboons. To exclude any order effects, the order of the conditions was balanced across individuals.

The control trials were conducted to examine whether subjects exhibited a laterality bias, that is, going on the same side on every trial. Furthermore, in the control trials of the 'Pebbles' condition, raisins were placed only under one plate to discover whether the subjects used other cues such as smell, sight or cues from the experimenter or the baiting procedure, which they did not $(47.5 \%$ correct).

Because it was suggested that the difference in performance might be due to the fact that in the Food condition, the choice stimuli had lesser associative strength because they changed in appearance while the items were fed to the monkeys (T Dickinson, personal communication), we ran a further set of control experiments with eight of the macaques (two subjects were 


\section{Chapter 3 - Quantity Discrimination}

excluded due to motivational problems). In the first control condition, all food items were taken away after the subject had made their choice ('Food away'), and then given to the monkeys while hidden in the experimenter's palm. In the second control condition ('Pebbles away'), we used pebbles as choice stimuli. After the subject made its choice, a pebble was removed each time when one of the food items underneath the plate was given to the monkey. To control for learning effects, we ran the initial 'Food' condition again. Overall, there was a slight increase in accuracy between the first and second sets of experiments in the 'Food' condition $174.1 \%$ correct). This increase was not significantly different $(P>0.2)$.

Statistics. We used a generalized linear mixed model implemented in the $\mathrm{R}$ statistical computing environment32. GLMM was implemented using the glmer function from the Ime4 package33. We used species (2 levels), condition (3 levels), absolute magnitude of the difference (4 levels) and ratio (11 levels) as fixed factors and subject as random factor. A MCMC procedure was used to approximate the significance levels of the parameter estimates. In the additional control experiments, we only tested macaques, and compared the performance in the 'Food away' versus the 'Pebbles away' conditions. To test the effect of experience, we compared the performance of the macaques in the initial and repeated 'Food' condition.

\section{Acknowledgments}

We thank Sara Jalali and Dennis Golm for help with the data collection. We are indebted to Uwe Schönmann and Annette Husung and all the animal caretakers for their help. We thank Christoph Teufel and Tony Dickinson for discussion and Sara Shettleworth and Andreas Nieder for helpful comments on an earlier version of the manuscript. All experiments followed the federal guidelines for the use of animals in research and were approved by the local ethical committee. This study was funded by the German Initiative of Excellence and the Leibniz Graduate School for Primate Neurobiology (Neuroprim). 


\section{CHAPTER 4}

\section{Food, Sex, and the Brain: Which Factors Influence Size Discrimination in Primates?}

\footnotetext{
Vanessa Schmitt ${ }^{1,2}$, Iris Kröger ${ }^{3}$, Dietmar Zinner ${ }^{1}$, Josep Call ${ }^{3}$, Julia Fischer ${ }^{1,2}$

${ }^{1}$ Cognitive Ethology Lab, German Primate Center, Göttingen, Germany

${ }^{2}$ Courant Research Centre Evolution of Social Behaviour, University of Göttingen, Germany

${ }^{3}$ Max Plank Institute for Evolutionary Anthropology, Leipzig, Germany
}

prepared for submission

Author Contributions

JC and DZ conceived the initial study; VS and JF extended it; IK and VS collected the data; VS, JC and IK analysed the data; VS and JF wrote the manuscript; all authors commented on the manuscript 


\title{
Chapter 4 - Size Discrimination
}

\begin{abstract}
Animals regularly face the problem to distinguish between different sized competitors, food items, or any traits conveying information of the risks and benefits for the animal. These challenges may exert differential selective pressures on species' abilities to discriminate objects of different size. In order to assess the ability for fine-grained size discrimination, we tested five different primate species, including great ape (chimpanzees, bonobos, gorillas) and two Old World monkey species (olive baboons, long-tailed macaques) in two-choice tests. The results are consistent with the view that dietary specializations drive species' discriminatory abilities. Species with a predominantly frugivorous diet (chimpanzees, bonobos, baboons, macaques) were able to discriminate three-dimensional cubes with a difference in size of only $10 \%$ (i.e. between cubes of $50 \mathrm{~mm}$ and $48 \mathrm{~mm}$ side length) in both simultaneous and successive presentations. Gorillas, a more folivorous species, did not discriminate between objects with $30 \%$ size difference (i.e. $6 \mathrm{~mm}$ ), but managed to distinguish between objects differing $60 \%$ in size. Presence of exaggerated sexual swellings, as an example for a trait in which changes in size is believed to convey information on ovulation probability and brain size, as a proxy for general cognitive abilities did not account for variation in performance. These findings highlight the importance of considering environmental factors in comparative studies, which might have influenced the evolution of perceptual and cognitive capacities.
\end{abstract}




\section{Introduction}

Animals are confronted with differently sized items such as foods, conspecifics or predators throughout their lives. The ability to discriminate items on the basis of their size is assumed to be highly advantageous. Sexual selection theory for example predicts that females should mate selectively with high-quality males and choose their mates according to signals that reliably indicate male quality (Kappeler \& van Schaik 2004). One predictor of male quality is body size, because it shows that (1) the male was actually able to accumulate enough nutrients and energy to grow to its respective size and (2) larger bodied males may have a higher resource holding potential and are more successful competitors (Trivers 1972; Andersson 1994). Choosing the larger male consequently may increase a females' reproductive success and her fitness. Recent playback studies in red deer revealed that females indeed prefer large males and use acoustic cues to infer males' body size in a mate choice context (Charlton et al. 2007). The ecological significance of size discrimination is also demonstrated in the behaviour of cowbirds. These brood parasites lay their eggs into the nests of other bird species, choosing the nest with eggs smaller than their own, so their young can outcompete the hatching nest mates (White et al. 2007). To discriminate between different sized objects animals may not only use visual but also tactile information. Studies in humans and monkeys have for example shown that they can choose the larger object only via haptic comparisons, i.e. touching the objects (Hille 2001; Kahrimanovic et al. 2011; see also Simon et al. 2006 showing size disrimination in bats using echolocation).

The importance to discriminate different sized objects may however differ among species, depending on factors such as ecology or mating system. Nonetheless, behavioural studies on visual size discrimination comparing the performance of different species and accounting for the possible influences of ecological factors, are rare and only few psychophysical studies investigated the actual abilities of animals in this domain (see Mishkin \& Hall 1955 for a brain lesion study in monkeys; Cloarec 1986 studying insects; see also the growing interest in studies on visual illusions, Suganuma et al. 2007; Tudusciuc \& Nieder 2010). This is quite surprising considering the amount of studies on quantity discrimination skills (see e.g. Nieder 2005 for a review), a generally similar ecological challenge. Instead, most studies interested in perceptual capacities do not examine the actual discrimination thresholds of animals, but use different sized objects to explore other cognitive features, such as relational learning (see e.g. Sarris et al. 2001; Hauf 2008). In their studies on relational matching Kennedy and colleagues (Kennedy \& Fragaszy 2008; Flemming \& Kennedy 2011) used objects of different size to test whether capuchin monkeys and chimpanzees are able to match a demonstrator's action to find hidden 
food. Whereas the chimpanzees performed well, only one capuchin mastered the task, supposedly showing species differences in analogical reasoning. However, as the size differences between the objects used were rather small, the results may be influenced by perceptual rather than cognitive differences (see also Bshary et al. 2011 for a discussion on incorporating perceptual characteristics in cognitive studies).

As nonhuman primates are such a diverse order, environmental factors may have differently influenced the evolution of the ability (and motivation) to attend to differences in size (see Shettleworth 2010b for examples on sensory adaptations in animals). In particular, specific aspects of their ecology, such as a frugivorous diet, may have facilitated size discrimination abilities as for example choosing the larger fruit item (which has a higher nutritional value) increases an animals' fitness. Furthermore, females of some Old World primate species exhibit exaggerated sexual swellings during their fertile phase, and their fluctuating size should encode information on ovulation probability, which in turn influences male sexual behaviour and malemale competition for matings (Zinner et al. 2002; Zinner et al. 2004). In such species there may be a premium on (males') ability to discriminate between swellings of different size. Moreover, brain size may influence general intelligence (Reader et al. 2011), such as decision-making abilities, which may promote more efficient choices in situations facing a judgment based on size. Comparing species differing in these aspects would help to conclude which factors may have influenced the evolution of size discrimination.

Species similarities may, however, also be due to phylogenetic relatedness, as a specific competence may be inherited by all species through common descent (MacLean et al. 2011; Nunn 2011). To distinguish between these different accounts, we tested five closely related primate species, which differed among others in brain size, diet, and the presence of exaggerated sexual swellings in females. Specifically, we included three great ape (chimpanzees, bonobos, gorillas) and two Old World monkey species (olive baboons, long-tailed macaques) in our study. The subjects were tested in two-choice tests in which they were rewarded for choosing the larger of two cubes, which were presented simultaneously. Because in real life, objects are not always fully visible at the same time, we included a second condition in which the two cubes were not shown simultaneously to the subjects, but in succession (increasing the time interval from $5 \mathrm{~s}$ to $10 \mathrm{~s}$ to $60 \mathrm{~s})$. We generated the following predictions: 1) If ecological factors like diet influenced the evolution of size discrimination abilities, then species relying on fruit should be able to discriminate between smaller size differences than folivorous species; 2) if female swelling size mainly influenced species capacities, then chimpanzees, bonobos and baboons should outperform long-tailed macaques and gorillas, as these exhibit much smaller 
swellings than the other species; 3 ) if brain size has an influence on the discrimination abilities of the species, then monkeys should perform worse than apes, as these possess much smaller brains corrected for body size (Jerison 1973) (see Table 1).

Table 1: Summary of the hypotheses and predictions regarding the size discrimination abilities of the tested species

\begin{tabular}{cl}
\hline Hypothesis & \multicolumn{1}{c}{ Predictions } \\
\hline Frugivory & Gorillas should perform worse than all other species \\
Swelling size & $\begin{array}{l}\text { Gorillas and long-tailed macaques should perform worse than } \\
\text { chimpanzees, bonobos and baboons }\end{array}$ \\
Brain size & $\begin{array}{l}\text { Long-tailed macaques and baboons should perform worse than } \\
\text { great apes }\end{array}$ \\
\hline
\end{tabular}

\section{Experiment 1: Small size discrimination}

In this experiment we tested, which size respectively volume differences of three-dimensional cubes the monkeys and apes were able to distinguish: first, when they were presented simultaneously; and second, when the cubes were only shown in succession.

\section{Methods}

\section{Subjects}

Apes

Five chimpanzees (Pan troglodytes), five bonobos (Pan paniscus) and eight gorillas (Gorilla gorilla) participated in this study -6 males and 12 females with an age of 7 to 28 years (Online Resource 1). All subjects were housed at the Wolfgang Koehler Primate Research Centre Leipzig, Germany. The apes lived in social groups and had access to indoor and outdoor enclosures. Subjects were individually tested in a familiar testing room (chimpanzees and gorillas) or in their sleeping cages (bonobos). Water was always available at libitum and subjects were not food deprived for testing. All apes were used to experimental testing situations.

\section{Monkeys}

Nine olive baboons (Papio anubis) and eight long-tailed macaques (Macaca fascicularis) - 6 males and 11 females with an age of 2 to 11 years - participated in this study (Table S1). One baboon (NS) dropped out of the study ahead of time because she was transferred to another facility. The long-tailed macaques (Macaca fascicularis) lived in a social group of 28 animals. The olive baboons (Papio anubis) lived in a social group of 11 animals. The monkeys were housed at 
the German Primate Center in Göttingen and had access to indoor (baboons: 17sqm, macaques: 40sqm) and outdoor areas (baboons: 81sqm, macaques: 141sqm).

Subjects were individually tested in their familiar indoor enclosure. Water was always available at libitum and subjects were not food deprived for testing. None of the baboons had experience in cognitive experiments, whereas the macaques had already participated in previous studies (Schmitt \& Fischer 2011; Schmitt et al. 2012)

\section{Materials}

A set of 9 equilateral cubes of different volumes (Table 2) was used. The cubes were built of pink cardboard and covered with transparent adhesive plastic film. One side of the cube was open so that the cubes could be placed over a food reward (grape or peanut). The cube with an edge length of $5 \mathrm{~cm}$ was set to represent $100 \%$ (Table 2 ).

A sliding table was used to place the cubes in front of the subjects. To do so, a sliding board was attached to a table so that the board could be moved horizontally. The table was attached with an iron mount in front of a plastic pane. Two cubes were placed on the right and left side of the sliding board. Two holes (apes: diameter $35 \mathrm{~mm}$, distance from centre to centre $0.56 \mathrm{~m}$; monkeys: diameter $15 \mathrm{~mm}$, distance $0.30 \mathrm{~m}$ ) in the plastic panel allowed the subjects to point with their fingers at the cubes. Additionally two blue plastic cups (height $75 \mathrm{~mm}$, diameter $90 \mathrm{~mm}$ ) were used to cover the pink cubes in the successive conditions. In addition, an occluder could be set up in front of the panel so that the subject was not able to watch the baiting of the cubes. All trials were videotaped.

Table 2: Cube set used to test the size discrimination abilities of the apes and monkeys.

\begin{tabular}{cccccccccc}
\hline Size & $140 \%$ & $130 \%$ & $120 \%$ & $110 \%$ & $100 \%$ & $95 \%$ & $90 \%$ & $80 \%$ & $70 \%$ \\
\hline $\begin{array}{c}\text { edge length } \\
\begin{array}{c}(\mathbf{m m}) \\
\text { Volume } \\
\left(\mathbf{m m}^{3}\right)\end{array}\end{array}$ & 58 & 56 & 54 & 52 & 50 & 49 & 48 & 46 & 44 \\
\hline
\end{tabular}

\section{Test Design}

\section{Simultaneous presentation}

Each subject was first tested in the simultaneous condition. Here every trial consisted of the following elements: the sliding table was removed from the panel and the occluder was positioned to hide the setup. The experimenter showed a food reward (grape or peanut) to the subject and then placed the reward on the sliding table where the subject was not longer able 
to see it. Then the experimenter showed the two cubes with the open side towards the subject so that it could see the cubes where empty. Next, the experimenter covered the reward with the larger cube and placed one cube to the right and the other to the left side of the sliding table (pseudorandomly, with the restriction that the reward should not appear on the same side for more than two consecutive trials but equally often left and right). The experimenter removed the occluder and pushed the table to the panel. The subject was allowed to choose one of the cubes by pointing at it through the holes in the panel. If the subject chose the bigger cube it received the reward, otherwise it received nothing, but was shown the place of the reward.

Each trial was repeated 12 times per session. If the subject chose 11 or 12 times the larger cube the session was scored as "passed". Each subject received a maximum of 12 sessions per volume difference.

Every subject started with a volume difference of $30 \%$ (Table 2). If the subject reached criterion twice with this volume difference (i.e. passed two sessions), the condition was scored as "passed" and the volume difference was decreased. If the subjects did not reach criterion within the 12 sessions, the condition was scored as 'failed' and the subject was not tested further. The volume difference was progressively decreased until the subject either failed the condition or reached the $5 \%$ volume difference condition. Afterwards the subject was tested using successive presentations of the stimuli.

All of the baboons failed in the start condition of $30 \%$ size difference. As, however, none of them had had prior experience in an experimental test situation, we increased the size difference to $100 \%$ (Table 3). The subjects who passed this condition continued with $80 \%, 60 \%$ and so on until they failed. The rest of the procedure was the same as for the other species.

\section{Successive presentation}

The procedure was the same as in the simultaneous presentation but additionally both cubes were covered with blue cups before the occluder was removed. Then each cup was lifted one after the other for 3 seconds so that the subject could see the cube underneath. The time span between the hiding of the first cup and the lifting of the other was increased incrementally from $5 \mathrm{~s}$ to $20 \mathrm{~s}$ and then $60 \mathrm{~s}$. At the time of choice both cubes were covered. Subjects were rewarded when they chose the cup underneath of which the larger cube was hidden.

The successive presentations started with the volume difference the subject had passed at last in the simultaneous presentations, that is, if a subject passed $10 \%$ in the simultaneous presentation but failed the $5 \%$ condition, it was first tested with $10 \%$ volume difference and a 
time span of 5 seconds in the successive presentation. If the subject passed the condition ( $>10 / 12$ correct in two sessions), the time span was extended to $20 \mathrm{~s}$ and afterwards to $60 \mathrm{~s}$. If a subject did not pass one of the time intervals the volume difference was increased (e.g. from $10 \%$ to $20 \%$ ) and the subject was tested with the respective time interval. If the subject passed now, the time interval was increased again until the 60s interval was reached. If it failed, the volume difference was increased further (e.g. from $20 \%$ to $30 \%$ ) until the subject passed the time interval or failed in all conditions.

The baboons received a slightly different procedure to account for the different cube combinations in the simultaneous condition. As for the other species the successive presentations started with the volume difference the baboon had passed at last in the simultaneous presentations. However, if a subject failed the 5-second delay with this size difference, we immediately increased the difference to $100 \%$ (see Table 3). If the baboon passed, we then stepwise decreased the size difference until the subject failed in a size difference. We then increased the time delay to $20 \mathrm{~s}$ and $60 \mathrm{~s}$ for the size difference the subject had passed at last. Again each subject received a maximum of 12 sessions per condition, with 12 experimental trials and 2 motivational trials (i.e. the cubes were shown simultaneously) per session.

Table 3: Cube combination for the apes and monkeys (only the baboons were tested with the $40 \%-100 \%$ volume differences)

\begin{tabular}{lcccccccc}
\hline $\begin{array}{l}\text { Volume } \\
\text { difference }\end{array}$ & $\mathbf{1 0 0 \%}$ & $\mathbf{8 0 \%}$ & $\mathbf{6 0 \%}$ & $\mathbf{4 0 \%}$ & $\mathbf{3 0 \%}$ & $\mathbf{2 0 \%}$ & $\mathbf{1 0 \%}$ & $\mathbf{5 \%}$ \\
\hline $\begin{array}{l}\text { Cube } \\
\text { combination }\end{array}$ & $58 \mathrm{~mm}$ & $56 \mathrm{~mm}$ & $54 \mathrm{~mm}$ & $52 \mathrm{~mm}$ & $50 \mathrm{~mm}$ & $50 \mathrm{~mm}$ & $50 \mathrm{~mm}$ & $50 \mathrm{~mm}$ \\
\hline
\end{tabular}

\section{Results}

\section{Simultaneous Discrimination}

Figure 1 presents the number of subjects that passed (or failed) the simultaneous discrimination as a function of sex and species. None of the gorillas learned to choose the larger of the two cubes, whereas 4 to 5 subjects of each other species did. In total 9 females and 9 males chose the larger cube successfully.

Excluding gorillas, the subjects were able to discriminate between alternatives that differed on average $20.6 \%$ in size $(S D=10.1$ ) (see Fig. 2 and Table 4). Two subjects were even able to discriminate reliably between cubes that differed only by $1 \mathrm{~mm}$ in edge length (i.e. $5 \%$ ). Neither $\operatorname{sex}(F(1,10)=1.99, p=.188)$, species $(F(3,10)=1.57, p=.256)$ or species $x \operatorname{sex}(F(3,10)=2.09, p$ $=.165)$ had any influence in the lowest discrimination point reached by the subjects. 


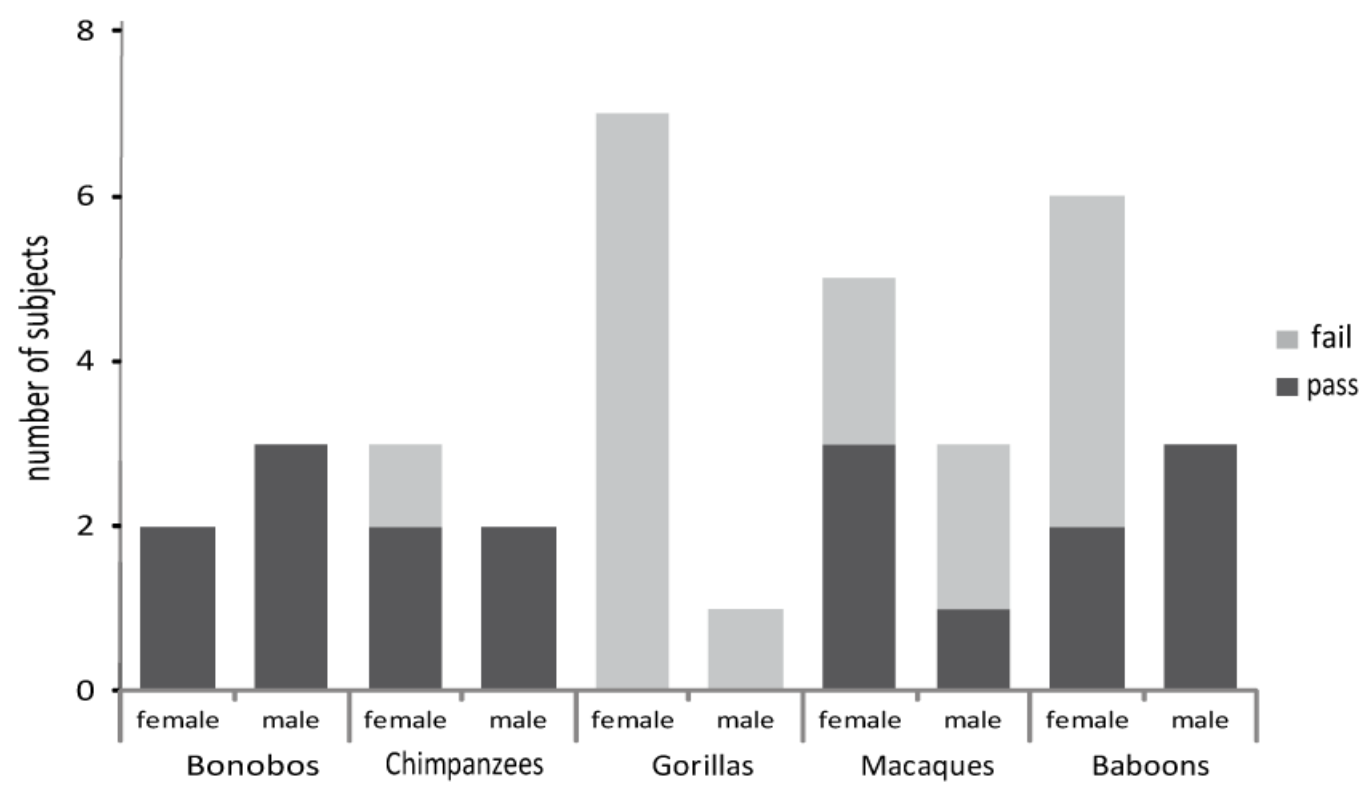

Fig.1: Number of subjects, which did and did not learn to choose the larger of two cubes in the simultaneous condition of Experiment 1.

\section{Successive Discrimination}

All subjects that had learned to choose the larger cube in the simultaneous presentation were still able to discriminate between the stimuli when these were presented in succession (except for one bonobo). After a 5-second delay subjects could discriminate between alternatives with an average difference of $18.13 \%$ in size $(S D=8.34)$. One macaque did not pass the subsequent 20s delay, but the other subjects were able to discriminate between approximately $18 \%$ size difference with 20 s and 60 s between the presentations of the cubes. There were no significant differences between the species $(F(3,7)=0.628, p=.619)$ or sexes $(F(1,7)=0.019, p=.895)$ in the successive conditions.

Comparing the two conditions, a two-way repeated measures ANOVA revealed a significant interaction between species and condition $(F(3,12)=3.91, p=.037)$. There were no significant differences between the simultaneous and successive condition for the bonobos, chimpanzees and macaques (Post hoc tests, all $p>.259$ ), but the baboons performed significantly better in the successive than on the simultaneous condition $(t=2.84 \mathrm{p}=.015)$. In contrast to the baboons, the other subjects were able to discriminate smaller size differences in the simultaneous than in the successive condition. 


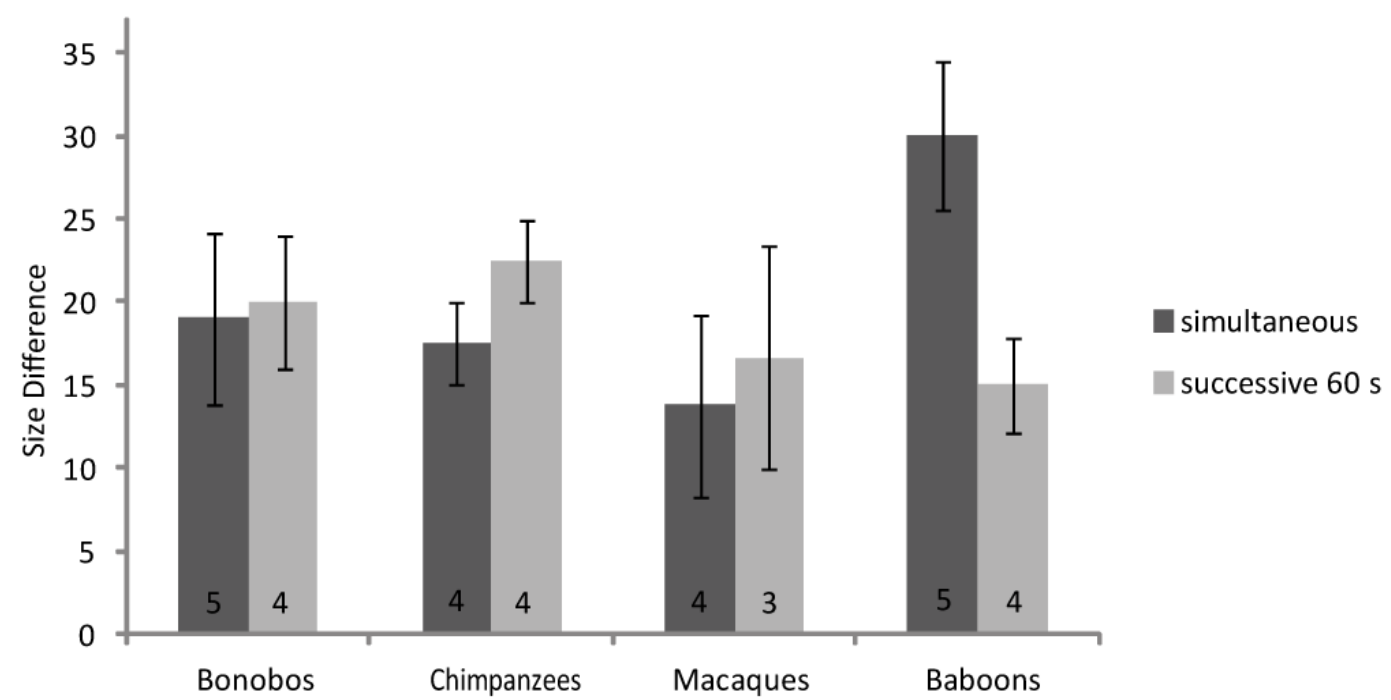

Fig. 2: Mean $( \pm S E)$ smallest size difference the subjects were able to discriminate, when the cubes were presented simultaneously and with a 60 -second delay. The values inside the bars indicate the number of subjects included in the condition.

Table 4: Smallest size difference (in \%) the subjects were able to discriminate in each condition. $f=$ failed the condition

\begin{tabular}{|c|c|c|c|c|}
\hline Condition & simultan & $5 s$ & $20 s$ & 60s \\
\hline \multicolumn{5}{|l|}{ Chimpanzee } \\
\hline Frodo & 20 & 20 & 20 & 20 \\
\hline Patrick & 20 & 20 & 20 & 20 \\
\hline Dorien & 20 & 30 & 30 & 30 \\
\hline Natascha & $f$ & & & \\
\hline Fraukje & 10 & 20 & 20 & 20 \\
\hline \multicolumn{5}{|l|}{ Bonobo } \\
\hline Joey & 30 & $f$ & & \\
\hline Limbuko & 10 & 10 & 10 & 20 \\
\hline Kuno & 5 & 10 & 10 & 10 \\
\hline Ulindi & 30 & 30 & 30 & 30 \\
\hline Yasa & 20 & 20 & 20 & 20 \\
\hline \multicolumn{5}{|l|}{ Gorilla } \\
\hline Gorgo & $f$ & & & \\
\hline Ndiki & $f$ & & & \\
\hline Bebe & $f$ & & & \\
\hline Viringika & $f$ & & & \\
\hline Bianka & $f$ & & & \\
\hline Hakuna & $f$ & & & \\
\hline Lena & $f$ & & & \\
\hline Ruby & $f$ & & & \\
\hline \multicolumn{5}{|l|}{ Baboon } \\
\hline Meister & 40 & 10 & 20 & 20 \\
\hline Jago & 40 & 10 & 10 & 10 \\
\hline Pünktchen & 20 & 10 & 10 & 10 \\
\hline Tröpfchen & $f$ & & & \\
\hline Nase & 30 & & & \\
\hline Schecki & $f$ & & & \\
\hline Brille & $f$ & & & \\
\hline Beinhaar & 20 & 20 & 20 & 20 \\
\hline Tiger & $f$ & & & \\
\hline
\end{tabular}




\begin{tabular}{ccccc}
\hline Condition & simultan & 5s & 20s & 60s \\
\hline $\begin{array}{c}\text { Macaque } \\
\text { Samson }\end{array}$ & $f$ & & & \\
Pit & 30 & 30 & 30 & 30 \\
Lenny & $f$ & & & \\
Sunny & $f$ & 10 & 10 & 10 \\
Maja & 10 & & & \\
Sally & $f$ & 30 & $f$ & 10 \\
Linda & 5 & 10 & 10 & \\
Sophie & 10 & & & \\
\hline
\end{tabular}

\section{Discussion}

The better performance of the baboons in the second, successive condition was probably due to their familiarization with the general setup und a better understanding of the test situation. Being able to choose the larger cube in the successive condition implicates a successful simultaneous discrimination. Indeed, in the motivational trials of the successive condition, when the cubes were presented simultaneously, all baboons choose the larger cube. In sum, the gorillas were outperformed by the other species regarding these fine grained size discrimination abilities, but there were no differences between apes or monkeys. To test whether gorillas have difficulties to discriminate between two different sized objects in general, we conducted an additional experiment with larger size differences between the stimuli.

\section{Experiment 2: Large size discrimination}

In this experiment we examined the abilities of gorillas, chimpanzees, bonobos and macaques to discriminate two objects with larger difference in size (about 60\%). Furthermore, we included a control condition to exclude that the subjects took any hint from the experimenter or baiting procedure to solve the task. (As the group of baboons was transferred to another facility we could not test them in this condition, but they had successfully discriminated $60 \%$ size differences in Experiment 1).

\section{Methods}

\section{Subjects}

Eight chimpanzees (Pan troglodytes; 3 males, 5 females), five bonobos (Pan paniscus; 3 males, 2 female), six gorillas (Gorilla gorilla, 2 males, 4 females) and seven long-tailed macaques (Macaca fascicularis, 4 males and 3 females) participated in the study. All apes were housed at the Wolfgang Köhler Research Center in Leipzig Zoo, the macaques where housed at the German Primate Center in Göttingen (s. Experiment 1 \& Table S1). 


\section{Materials}

The apparatus was the same as described above. Instead of the cubes two different pairs of opaque containers were placed on the platform. An occluder was used to hide the baiting from the monkeys. There were two sets of containers:

Size: Two white plastic plant pots identical in shape but differing in size (Set 1: $90 \mathrm{~mm}$ high $\mathrm{x}$ $110 \mathrm{~mm}$ in diameter vs. $120 \mathrm{~mm}$ high $\times 140 \mathrm{~mm}$ in diameter; Set 2: $100 \mathrm{~mm}$ high $\times 120 \mathrm{~mm}$ in diameter vs. $140 \mathrm{~mm}$ high $\times 160 \mathrm{~mm}$ in diameter). The larger pots were approximately $60 \%$ larger in volume than the smaller pots.

Control: Two green or two orange plastic cups of identical size $190 \mathrm{~mm}$ high and $70 \mathrm{~mm}$ in diameter) and shape.

\section{Procedure}

The experimenter put up the occluder, baited the larger pot with a reward and placed the pots to the left and right side on the table. The occluder was lifted and the subject was allowed to choose. If it chose the larger pot it received the reward, if it chose the smaller one it received nothing but was shown the place of the reward. The position of the baited object was pseudorandomized with the restriction that the reward should not appear on the same side for more than two consecutive trials, but equally often left and right.

All subjects participated in a total of 96 trials in eight 12 -trial sessions; four control trials were randomly interspersed within each session. Starting with Set 1 or Set 2 was randomised and balanced across individuals. Each individual received one or two sessions per day.

\section{Results}

A repeated measures ANOVA (with stimulus set as random factor and species as dependent factor) revealed no significant differences between the species tested $(F(3,20)=2.729, p=.217)$. All four species chose the larger pot significantly above chance (Wilcoxon-test: bonobos $p=$ .043 ; chimpanzees $p=.012$; macaques $p=.018$; gorillas $p=.046$ ) (see Fig.3). In the control condition none of the subjects performed above chance, making it unlikely that they took any hint from the baiting procedure or the experimenter to solve the task. Macaques, bonobos and gorillas chose the correct cup even less often than expected by chance (Wilcoxon-test: macaques $p=.063$; bonobos $p=.079$; gorilla $p=.249$; chimpanzees $p=.916$ ). 


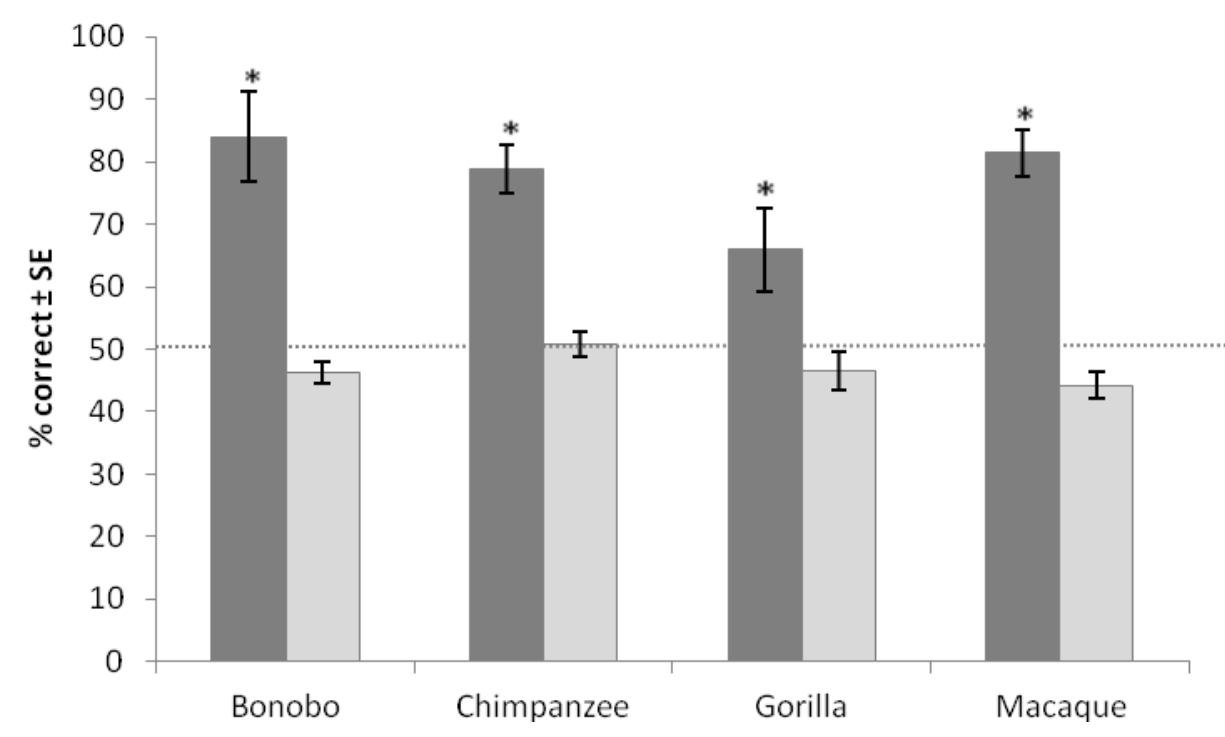

Fig. 3: Percent of correct responses of the apes and monkeys in the test condition (dark grey) and the control condition (light grey). The dotted line represents the chance level. ${ }^{*} p<.05$

\section{General Discussion}

Gorillas performed significantly worse in discriminating small size (volume) differences of two cubes than chimpanzees, bonobos, baboons and macaques. On average these were all able to recognize differences in volume which were only $20 \%$, and two subjects even discriminated between $5 \%$ size differences, i.e. $1 \mathrm{~mm}$ difference in side length. In the control experiment (Exp. 2), when the size difference was large, the gorillas were able to discriminate between the stimuli and performed only slightly worse than the other species, demonstrating that they probably do not have lower discriminatory abilities in general, but only problems to discriminate between subtle differences in size. That gorillas do not generally fare worse than the other great apes was also found in other studies (for a review on primate cognition see Tomasello \& Call 1997).

With the exception of gorillas, all species performed on the same discriminatory level in Experiment 1. There were no significant differences between chimpanzees, bonobos, baboons and macaques, suggesting that presence and size of sexual swellings may not have significantly influenced the evolution of species discriminatory abilities. Long-tailed macaques exhibit much smaller sexual swellings than baboons or chimpanzees (Engelhardt et al. 2004; Malaivijitnond et al. 2007a), but were able to discriminate the same size differences or even smaller ones (none of the baboons or chimpanzees passed the $5 \%$ size difference, but one macaque did). Regardless of this negative result, our experiments showed that males are able to notice size differences of about $20 \%$ and less, which corresponds to the observed changes in the period of maximum swellings in female chimpanzees (Deschner et al. 2004). Thus, the good size 


\section{Chapter 4 - Size Discrimination}

discrimination abilities, which may have evolved in response to frugivory, can be put to use in the context of sexual selection, where males need to be able to discriminate between females based on different swelling sizes.

Similarly, brain size did not have an influence on the species performances. Baboons and macaques, having smaller brains than apes (Jerison 1973), performed as well as chimpanzees and bonobos, refuting the assumption that larger brains enhance the ability to discriminate differently sized objects at this scale. The fact that the baboons had to be trained with a $100 \%$ size difference at the beginning may well be due to their lack of experience. None of the animals had ever participated in an experiment before and they had to get used to the testing situation itself to understand the task. Our results further indicate that monkeys and apes were both able to form mental representations of the cubes as they were able to pick the larger cube even after a 60 seconds delay, suggesting that they compared the objects internally (see also Schmitt \& Fischer 2011), a capacity which is often only assigned to apes (Byrne 2000). These findings corroborate a recent study, which also found no significant differences between apes and monkeys in a variety of cognitive tasks (Schmitt et al. 2012) challenging the view of a deep cognitive split between monkeys and apes (see also Amici et al. 2010).

The results suggest that ecological factors, like a frugivorous diet, primarily promoted the evolution of size discrimination abilities in primates. The fruit eating species outperformed the folivorous species. Being able to choose the larger of two fruit items has a substantial influence on an animals' fitness and evolution may have favoured subjects, which could pick the larger fruit item when competing with conspecifics. However, we could test only one folivorous species and future studies should examine the capacities of other folivorous primates to assess whether diet is indeed the determining factor. The fact that colour vision probably also evolved in response to frugivory demonstrates, however, that diet can have a significant influence on species' perceptual abilities (Osorio \& Vorobyev 1996).

Taken together our study shows that primates are able to notice and remember subtle differences between two objects, even after successive presentation, but that this capacity seems to differ significantly between species. Visual size discrimination seems to be largely influenced by environmental factors, such as diet, with no apparent influence of brain size. These findings emphasize the importance of considering environmental factors in comparative studies as these probably influenced the evolution of perceptual and also cognitive capacities. 


\section{Acknowledgments}

We thank Uwe Schönmann and Annette Husung of the German Primate Center and all the animal caretakers for their help. This study was funded by the German Initiative of Excellence and the Leibniz Graduate School for Primate Neurobiology (Neuroprim).

\section{Ethical standards}

All experiments followed the federal guidelines for the use of animals in research and were approved by the local ethical committee. 


\section{Supplement}

Table S1. Name, gender, age, rearing history, and study participation

\begin{tabular}{|c|c|c|c|}
\hline Subject & Gender & Age (years) & Study participation \\
\hline \multicolumn{4}{|l|}{ Chimpanzee } \\
\hline Robert & M & 30 & 2 \\
\hline Frodo & M & 12 & 1,2 \\
\hline Patrick & M & 9 & 1,2 \\
\hline Riet & $\mathrm{F}$ & 28 & 2 \\
\hline Dorien & $\mathrm{F}$ & 25 & 1,2 \\
\hline Sandra & $\mathrm{F}$ & 13 & 2 \\
\hline Jahaga & $\mathrm{F}$ & 13 & 2 \\
\hline Fifi & $\mathrm{F}$ & 13 & 2 \\
\hline Natascha & $\mathrm{F}$ & unknown & 1 \\
\hline Fraukje & $\mathrm{F}$ & unknown & 1 \\
\hline \multicolumn{4}{|l|}{ Bonobo } \\
\hline Joey & M & 23 & 1,2 \\
\hline Limbuko & M & 10 & 1,2 \\
\hline Kuno & M & 8 & 1,2 \\
\hline Ulindi & $\mathrm{F}$ & 12 & 1,2 \\
\hline Yasa & $\mathrm{F}$ & 9 & 1 \\
\hline \multicolumn{4}{|l|}{ Gorilla } \\
\hline Gorgo & M & 25 & 1,2 \\
\hline Nkwango & M & 9 & 2 \\
\hline Ndiki & $\mathrm{F}$ & 28 & 1,2 \\
\hline Bebe & $\mathrm{F}$ & 25 & 1,2 \\
\hline Viringika & $\mathrm{F}$ & 11 & 1,2 \\
\hline Bianka & $\mathrm{F}$ & unknown & 1 \\
\hline Hakuna & $\mathrm{F}$ & unknown & 1 \\
\hline Lena & $\mathrm{F}$ & unknown & 1 \\
\hline Ruby & $\mathrm{F}$ & unknown & 1,2 \\
\hline \multicolumn{4}{|l|}{ Baboon } \\
\hline Meister & M & 7 & 1 \\
\hline Jago & M & 2 & 1 \\
\hline Pünktchen & M & 2 & 1 \\
\hline Tröpfchen & $\mathrm{F}$ & 11 & 1 \\
\hline Nase & $\mathrm{F}$ & 7 & 1 \\
\hline Schecki & $\mathrm{F}$ & 6 & 1 \\
\hline Brille & $\mathrm{F}$ & 5 & 1 \\
\hline Beinhaar & $\mathrm{F}$ & 3 & 1 \\
\hline Tiger & $\mathrm{F}$ & 3 & 1 \\
\hline \multicolumn{4}{|l|}{ Macaque } \\
\hline Popey & M & 4 & 2 \\
\hline Samson & M & 3 & 1,2 \\
\hline Pit & M & 3 & 1,2 \\
\hline Lenny & M & 2 & 1,2 \\
\hline Sunny & $\mathrm{F}$ & 9 & 1 \\
\hline Maja & $\mathrm{F}$ & 4 & 1 \\
\hline Sally & $\mathrm{F}$ & 4 & 1,2 \\
\hline Selina & $\mathrm{F}$ & 3 & 2 \\
\hline Linda & $\mathrm{F}$ & 2 & 1 \\
\hline Sophie & $\mathrm{F}$ & 2 & 1,2 \\
\hline
\end{tabular}




\title{
CHAPTER 5
}

\section{Now you see me, now you don't - Social Inhibition of the Use of Pointing Cues?}

\author{
Vanessa Schmitt ${ }^{1,2}$, Christian Schloegl ${ }^{1,2}$, Julia Fischer ${ }^{1,2}$ \\ ${ }^{1}$ Cognitive Ethology Lab, German Primate Center, Göttingen, Germany \\ ${ }^{2}$ Courant Research Centre Evolution of Social Behaviour, University of Göttingen, \\ Germany
}

Submitted

Author Contributions

VS, JF and CS designed the study; VS collected the data; VS analysed the data; all authors contributed substantively to the writing and editing of the final draft 


\title{
Chapter 4 - Understanding Pointing
}

\begin{abstract}
The cultural intelligence hypothesis postulates that humans evolved special skills for cooperative communication. Supporting this assumption, nonhuman primates typically fail to use human communicative cues like pointing to find rewards. In contrast, dogs (but not wolves) also can make use of human cues, which was explained as a result of domestication. More puzzling is the fact that nonhuman primates apparently do not even learn to use pointing cues. Here we investigated whether the presence of the experimenter impairs learning in a group of long-tailed macaques. We found that the monkeys successfully used pointing cues in an objectchoice paradigm when only the arm of the experimenter (72\% correct), a doll's arm (78\%) or a stick $(70 \%)$ was visible, compared to the baseline condition with the experimenter visible $(61 \%)$. Performance was significantly better when the distance between stimulus and target was close. Intriguingly, after these experiments the monkeys' performance was also significantly improved with the experimenter being visible (70\%). Apparently, the monkeys were first distracted by the perception of the experimenter, but then learned to use the cue. These findings highlight the importance of test conditions, and call into question some of the assumptions about speciesspecific differences in the ability to comprehend communicative gestures.
\end{abstract}

Keywords: Communication; Human cues; Pointing; Primates; Social Inhibition; Local Enhancement 


\section{Introduction}

The pointing gesture - pointing to an object with the extended index finger - is assumed to be a uniquely human communicative action (Tomasello 2008; see Miklósi \& Soproni 2006; Mulcahy \& Hedge 2012 for reviews on pointing) and is suggested to rely on our special cooperative nature, which further enables cultural learning, imitation and the emergence of language (cultural intelligence hypothesis, Herrmann et al. 2007; Tomasello 2008). Dogs are also able to use this cue when a person points to the location of a hidden reward (Hare et al. 2002). Apes and monkeys, in contrast, generally failed in this test paradigm (Tomasello 2008; Mulcahy \& Hedge 2012). This led to the hypothesis that through domestication, dogs acquired the sociocognitive skills to respond to human communicative gestures (Hare et al. 2002); but it remains controversial whether dogs understand the communicative intention of pointing or whether their performance is due to rapid learning (Reid 2009; Udell et al. 2010).

While nonhuman primates may not understand the communicative intent of the signaler (Tomasello 2008), it is puzzling that they even have difficulties to learn the significance of the pointing cue, while they do learn to comprehend acoustic cues in social contexts (Fischer et al. 2000) or arbitrary cues (e.g. cup color) in object choice paradigms (Schmitt \& Fischer 2009); furthermore, they also reliably follow the gaze of conspecifics (Teufel et al. 2010) and human experimenters (Emery 2000). So why is it so difficult for primates to make use of the pointing cue?

To address this question, we set out to test three non-mutually exclusive hypotheses regarding the use of the pointing cue. First, the monkeys may be distracted by the experimenters' body and face in the normal pointing paradigm. Direct gazing is often a threat gesture in animals (Emery 2000) and previous studies suggest that especially primates may see humans more as competitors than as cooperation partners (Hare \& Tomasello 2004). Thus, we 'removed' the body of the experimenter by placing her behind a curtain with only the pointing arm being visible to the subjects. If the presence of the body/face was a problem, the monkeys should perform better with the experimenter standing behind the curtain. Possibly though, the monkeys still linked the arm to the presence of the experimenter. We therefore also used a doll's arm and a stick to deliver the pointing cues. If the degree of abstractness enhances performance, we would predict that the monkeys' performance is best in the 'stick' condition, and intermediate in the 'doll' condition. Second, a considerable effect of local enhancement (distance between the cue and the cup) regardless of condition, would further suggest that the animals do not take the social aspect - and thus the communicative intent - into account. Third, the absence of the experimenter may facilitate general learning to understand the pointing cue. 
In this case, we would predict that the performance in a re-test in the standard object-choice task with the experimenter present after the completion should be significantly better.

\section{Material and Methods}

\section{Subjects}

We tested 10 long-tailed macaques (Macaca fascicularis) - 4 males and 6 females aged 2 to 8 years- at the German Primate Center in Göttingen (one subject did not finish the study because of health problems). The subjects were individually tested in their familiar indoor cages and were not food deprived for testing. All subjects had already participated in a battery of tasks assessing their socio- and physico-cognitive capacities (Schmitt \& Fischer 2011; Schmitt et al. 2012). Here they had also been tested in a pointing task, however, only few trials had been conducted and none of the animals had performed above chance.

\section{Testing apparatus}

Two blue opaque cups $(\varnothing 5 \mathrm{~cm} \times 5 \mathrm{~cm}$ height) were used to hide the reward (raisins or peanuts). These were fixed to a sliding board (distance $30 \mathrm{~cm}$ ) so that the monkeys could lift them by tilting them back (like a high-joint). The sliding board was attached to a fixed table (length $55 \mathrm{~cm}$, width $30 \mathrm{~cm}$ ) so that the sliding board could be moved horizontally. The table was attached to a plastic panel, which had two oval openings at the outer sites $(5.5 \mathrm{~cm} \times 2 \mathrm{~cm}$, distance $30 \mathrm{~cm}$ ) to allow the subjects to put one hand through and reach for a cup.

To hide the experimenter we put up a large black curtain $(2 \mathrm{~m} \times 1.50 \mathrm{~m})$ in front of the testing apparatus (see Figure S1). We cut a small slot into the middle of the curtain to allow inserting an arm or stick to point at a cup. Furthermore, we attached a baton to the end of the sliding board, which passed through the curtain and allowed to move the table back and forth without stepping in front of the curtain.

To point at the cups we either used the arm of the experimenter, a grey coloured stick $(\varnothing$ $4 \mathrm{~cm} \times 80 \mathrm{~cm}$ length) or a left and right arm of a normal fashion mannequin $(\varnothing 30 \mathrm{~cm} \times 80 \mathrm{~cm}$ length). It was possible to lower down an occluder of black cardboard $(70 \mathrm{~cm} \times 100 \mathrm{~m})$ hanging from the ceiling from behind the curtain to prevent the subject from seeing the baiting procedure. All sessions were videotaped (Sony DCR-HC90E).

\section{Experimental Procedure}

The experimenter baited one of two opaque cups, which were fixed on a sliding table, behind an occluder, which prevented the subjects from seeing the experimenter. In the test conditions she then stepped back behind a large curtain, remotely lifted the occluder and put 
her arm, a stick, or the arm of a mannequin doll through a slot in the middle of the curtain to point at the baited cup. She always used the arm contralateral to the baited cup, i.e. the left arm to point at the right cup and vice versa (also called cross pointing, Miklósi \& Soproni 2006). After approximately 3 seconds she pushed the sliding table, while still pointing, to the monkey, which then could choose one of the cups. In the baseline and the re-test condition the experimenter did not step behind the curtain and only pointed with her own arm at the cup. Half of the animals first passed all conditions with proximal cueing $(5-10 \mathrm{~cm}$ between cup and finger) and then received distal cueing $(30-40 \mathrm{~cm})$, the others received the opposite order.

\section{Conditions and Design}

\section{Baseline}

The experimenter stood in front of the curtain, pointed with her arm at the baited cup and gazed straight forward, not looking at the monkey or the cups. Each subject received two 9-trial sessions (18 trials in total) with proximal cueing and the two sessions with distal cueing in randomized order (one monkey passed only the proximal condition due to health problems).

\section{Test}

The experimenter stood behind the curtain throughout the experiment and either put her own arm (human), the arm of a doll (doll), or a stick (stick) through the curtain to point at the baited cup. Each subject received six 9-trial sessions (54 trials in total) with proximal and six sessions with distal cueing (one monkey passed only the proximal conditions). Each session contained 3 trials of each test condition (i.e. human, stick, doll) resulting in 18 trials per condition for the distal and for the proximal variant each. Conditions were randomised but balanced within and across individuals.

Re-Test

After the completion of all experimental conditions, all subjects were re-tested in the baseline condition with the experimenter present. Each subjects received two 9-trial sessions with proximal and distal cueing each.

\section{Control}

To control whether the animals used any cues like smell to find the reward we administered a control condition in which the experimenter baited one cup behind the occluder, lifted it, but then gave no cue to the correct location. Each subject received two 9-trial sessions. 


\section{Statistics}

We conducted a two-way repeated measures ANOVA to test whether the monkeys' performance differed between the four different conditions (baseline, doll, human, stick) or between the different distances between the cue and the cup (i.e., proximal vs. distal), and whether there was a significant interaction between these two factors (see Table S1 for the results). We conducted Post hoc tests (Holm-Sidak method) in case of a significant result. To test whether the monkeys improved in the re-test condition we compared these results to the baseline condition with proximal cueing using a paired t-test (data were normally distributed).

\section{Results}

All subjects performed at chance level in the control condition (51.1\% correct) and thus did not use any cue like smell or sight to find the reward. In the baseline and test conditions a twoway repeated measures ANOVA revealed a significant interaction between distance and condition $(F(3,24)=5.56, p=.005)$. This interaction was due to the fact that the subjects performed near chance level in the baseline condition at both distances, while in the other conditions they generally performed better in the proximal than the distal condition (Figure 1). For the post-hoc test, we thus compared the performances in the proximate conditions only, which revealed a significant difference between the baseline and doll and the baseline and human condition (Results pair-wise comparisons; doll vs. baseline: $p<.001$; human vs. baseline: $p=.039$; stick vs. baseline: $p=.114$ ) but no differences between the human and the doll condition and the stick and the other two conditions (human vs. doll: $p=.380$; stick vs. human: $p=.595 ;$ stick vs. doll: $p=.218$ ).

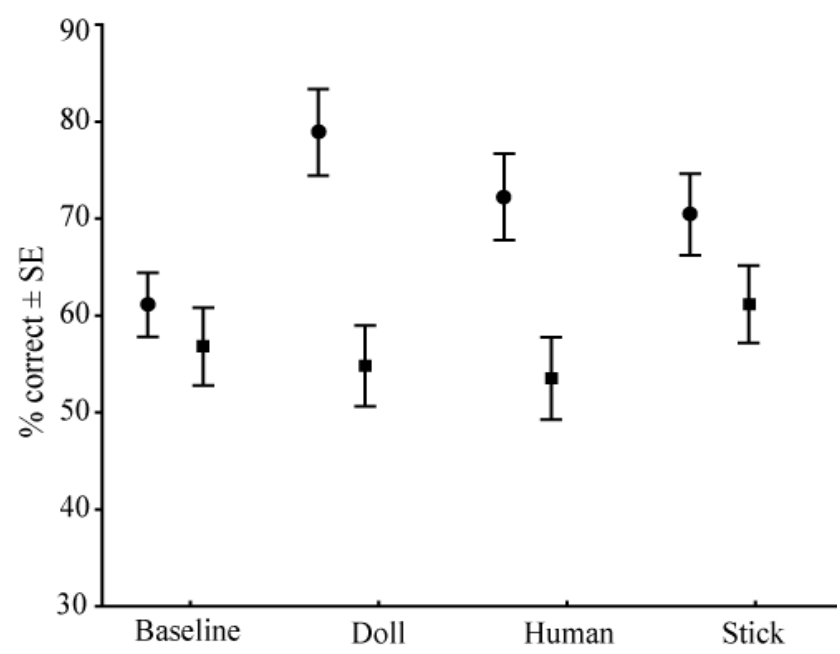

Figure 1: Percent of correct trials in the proximal (circle) and distal (square) conditions (means and standard error of means). Performance in the proximal Doll and Human condition was significantly better than in the proximal Baseline condition. 
When we repeated the baseline test at the end of the experiment the monkeys' performance improved significantly to $70.4 \%$ correct responses with proximal cueing compared to the baseline condition ( $t=3.41, p=.009$ ) (Figure 2).

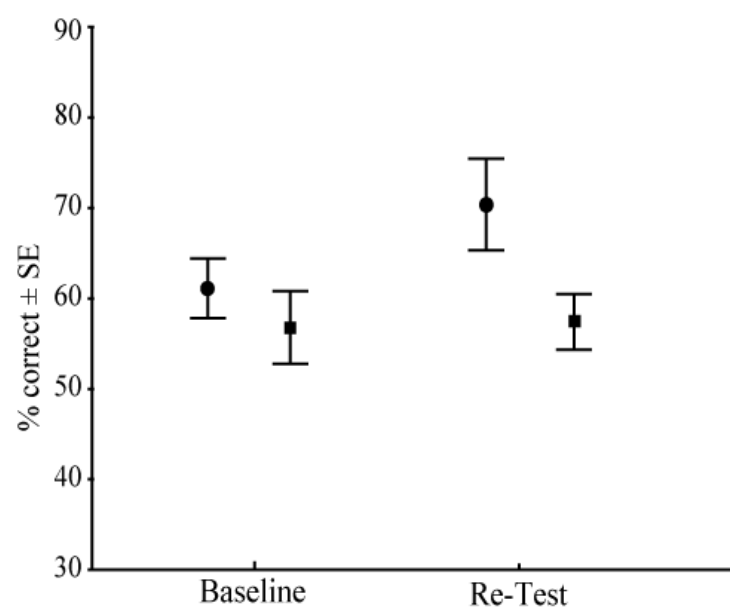

Figure 2: Mean percent of correct trials in the Baseline and Re-test (circle:proximal; square: distal). Performance in the proximal Re-Test condition was significantly better than in the proximal Baseline condition.

\section{Discussion}

Our results show that primates are able to use pointing and suggest that the presence of the experimenter indeed distracted them in normal pointing tests. Whether this is due to fear as they perceive the experimenter as a competitor or whether the cue is more conspicuous when the rest of the person stands behind a curtain remains to be tested. Furthermore, which kind of cue used to point at the correct location (human, doll, stick) did not have an influence on the monkeys' performance, as long as the distance between the stimulus and the target was close. This suggests that for the use of a human pointing gesture a special understanding of its communicative intent is not necessary. Instead, local enhancement may account for much of the monkeys' performance. If they had understood the communicative intent, they should have performed similarly in the distal and the proximal condition, but that was not the case. It remains a question of empirical investigation whether the monkeys would also learn to use a distal pointing cue when subjected to extensive training (so far only proximal cues have been trained successfully, Anderson et al. 1996). In children, it is also not fully clear to what extent learning accounts for the initial utilisation of pointing. Twelve month old infants look in the direction of the pointing, but only 15-months olds show some understanding of the communicative intent (Morissette et al. 1995; Behne et al. 2005).

Our results further suggest that social inhibition may explain the failure of primates to use human cues. When the experimenter hid behind the curtain the performance improved 


\section{Chapter 4 - Understanding Pointing}

considerably, supporting the hypothesis that the monkeys might have been afraid of the experimenters' face and eyes. Hiding these body parts seemed to reduce the monkeys' fear and allowed them to concentrate on the pointing cue. This finding may also shed new light on the outstanding performance of dogs. Some researchers suggested that through domestication dogs did not acquire special socio-cognitive skills but that learning was facilitated, especially through the reduction of fear (Reid 2009; Udell et al. 2010) and an increased tolerance to the sight of humans (what may also be the case for enculturated apes, Call \& Tomasello 1994). In conclusion, dogs may not learn to use human cues faster than primates but may be just better adapted to the situation (see also a recent study on the limits of dogs and children to use a pointing cue, Lakatos et al. 2009).

In conclusion, social inhibition seems to constrain learning when animals are confronted with human pointing (a recent review showed that in only three out of seventy experiments on pointing learning effects were observed, Mulcahy \& Hedge 2012). Furthermore, simple local enhancement was sufficient to associate the cue with the baited cup. There was no difference in performance concerning social vs. non-social cues suggesting that the social aspect may not play a major role in the acquisition of this association. Possibly, children and dogs also use associative learning first, which then scaffolds higher cognition.

\section{Ethical Standards}

All experiments were performed in accordance with the NRC Guide for the Care and Use of Laboratory Animals and the European Directive 2010/63/EU on the protection of animals used for scientific purposes. In accordance with the German Animal Welfare Act and corresponding section for animals used for scientific purposes, the study approval was checked by the responsible Animal Welfare Officer of the German Primate Center.

\section{Acknowledgements}

We thank Uwe Schönmann and Annette Husung and all the animal caretakers for their help. This study was funded by the German Initiative of Excellence and the Leibniz Graduate School for Primate Neurobiology (Neuroprim). CS was supported by the Leibniz Graduate School "Foundations of Primate Social Behaviour". 


\section{Supplementary Information}

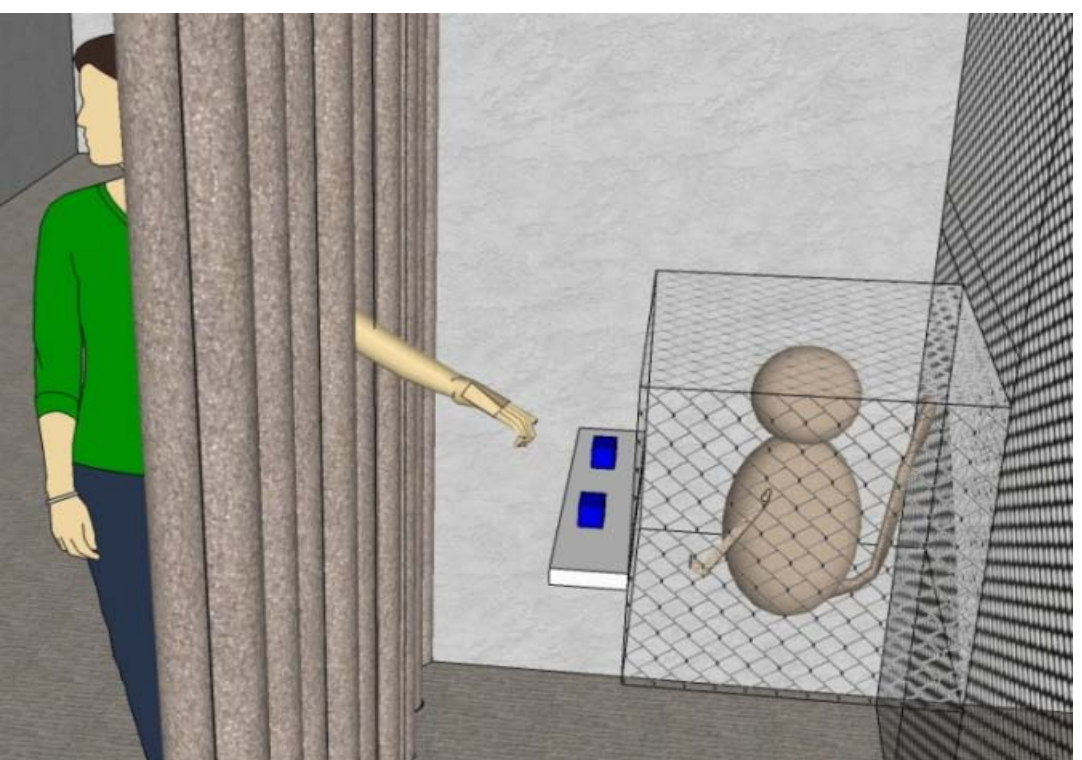

Figure S1: Simplified drawing of the test situation. The experimenter stood behind a curtain and only the arm was visible to the subject.

Table S1: Results of the two-way repeated measures ANOVA

\begin{tabular}{llllll}
\hline $\begin{array}{l}\text { Source of } \\
\text { Variation }\end{array}$ & DF & SS & MS & F & $\mathrm{p}$ \\
\hline Distance & $\mathbf{1}$ & $\mathbf{0 . 4 0 5}$ & $\mathbf{0 . 4 0 5}$ & $\mathbf{2 1 . 2 9 2}$ & $\mathbf{0 . 0 0 2}$ \\
Condition & 3 & 0.0837 & 0.0279 & 2.044 & 0.135 \\
Dist x Cond & $\mathbf{3}$ & $\mathbf{0 . 1 3 0}$ & $\mathbf{0 . 0 4 3 4}$ & $\mathbf{5 . 5 6 2}$ & $\mathbf{0 . 0 0 5}$ \\
\hline
\end{tabular}




\section{General Discussion}

In this dissertation I described a variety of cognitive experiments suggesting that monkeys and apes possess more similar skills than previously expected both in the social and physical domain. In addition, my experiments provided further insights into the cognitive mechanisms underlying the performances of the monkeys and the evolutionary pressures that might have shaped specific capabilities. In this general discussion, I will now 1) briefly revisit the main findings of the experiments and discuss their implications for the previously suggested monkeyape dichotomy, 2) critically consider the distinction of social and physical cognition, 3) discuss the difficulty of explaining observed behaviour in animals and 4) humans, 5) stress the problems of comparing different species, and finally 6) summarize additional studies conducted with the monkeys and make suggestions for future research.

\subsection{Monkeys vs. Apes, it's not that simple}

To obtain a better understanding of the evolution of (human) intelligence it is essential to identify which traits are derived in the course of a species' evolution and which traits are shared between species having a common ancestor. As brain size tends to increase from monkeys to apes to humans, it is suggested that intelligence similarly increases in the same fashion. One fundamental question is whether the largest "cognitive gap" is between humans and all other primates, or between the hominoids (i.e. apes and humans) and the rest of the primates, including Old World monkeys (see also Figure 1.1 in the Introduction for an illustration).

The experiments of the PCTB (Chapter 2) and the size discrimination study (Chapter 4) suggest that the cognitive differences between apes and monkeys are not as clear-cut as had been claimed by some researchers (e.g. Deaner et al. 2006; Dunbar \& Shultz 2007). In both studies the monkeys were not outperformed by apes, supporting the findings of a recent study examining physico-cognitive skills of different primate species (Amici et al. 2010). Here the authors compared the performance of three monkey species (spider monkeys, capuchin monkeys, long-tailed macaques) and all four great ape species in spatial displacement and causal relations tasks and also found no support for a clear-cut difference between apes and monkeys.

The assumption that apes possess higher general intelligence than monkeys is therefore not supported by our comparative analyses. But what about the more domain-specific hypotheses, especially those regarding social skills? In the experiments described in Chapter 2 we found no indication that the socio-cognitive capacities increased from monkeys to apes. In contrast, in 
some tasks the monkeys performed even better than the apes, for instance the macaques followed the human gaze significantly more often than the apes. However, as discussed in Chapter 2 this does not imply that monkeys possess better social skills than apes, as most of the tasked could be solved without the attribution of mental states. Indeed, the results of the pointing study reported in Chapter 5 suggest that monkeys do not understand the communicative intentions of humans, but rather solved the tasks by using physical principals such as local enhancement.

The hypotheses described in the Introduction therefore do not seem to explain the monkeyape distinction in general. The social or more specifically cultural intelligence hypotheses appear to apply only to the human-ape dichotomy. As Herrmann and colleagues (2007) found, human children excelled specifically in the social tasks. Concerning apes and monkeys these differences were not particularly straightforward in our tests. These results question the direct relation between brain size and cognition and the value of meta-analyses incorporating results of different studies using different methods. Although such analyses have revealed seemingly clear distinctions between the taxa, the fact that different analyses corroborate different hypotheses makes them even more disputable. For example, as mentioned in the Introduction Deaner and colleagues (2006) concluded from their meta-analyses that apes outcompete all other lineages, whereas Reader and colleagues (2011) conclude that high intelligence independently evolved at least four times in the primate order.

Nonetheless, although the performances of apes and monkeys did not significantly differ in our tests, this does not imply that their cognitive abilities are generally similar either. For example, only apes show some signs of self-recognition in front of a mirror. Chimpanzees display a variety of self-directed behaviours, such as inspecting parts of their body that could not be seen without a mirror, such as their teeth or ano-genital region, and also pass the socalled "mark test" (i.e. a coloured spot is put on the subject's head, being only visible when looking into a mirror, Gallup 1970; Shettleworth 2010b; see Anderson \& Gallup 2011 for a recent review). Orangutans and bonobos were also observed to respond to mirrors in such a way (but gorillas were not, Gallup et al. 2002). Monkeys in contrast do not show such reactions. In the majority of cases monkeys treat their reflections as a conspecific and perform social responses, such as threatening or submissive gestures. Although these reactions diminish over time, when moving the mirror to a new location the monkeys behaved as they had never seen the mirror before (Suarez \& Gallup 1986). Anderson and Gallup (2011, p. 2) therefore concluded: "the weight of evidence supports the view that the ability to direct one's attention to the self involves a qualitative cognitive shift, one that has occurred only recently in primate 
evolutionary history and in relatively few species." Although, whether the reactions of apes reveal human-like self-awareness is controversial and maybe better described as "mirror-guided body inspection" (Heyes 1994; Shettleworth 2010b), significant differences in the responses of monkeys and apes do exist.

Furthermore, until today only apes have been successfully taught to use and understand some rudimentary forms of human language (only regarding nonhuman primates; in fact other species seem also to understand some aspects of human language, as for example parrots, Pepperberg 1990; and dogs, Kaminski et al. 2004). After realizing that apes are not able to voluntarily control their utterances and talk (studies by Kelloggs and Hayes in the 1930s and 1940s), and using sign-language showed only slight and controversial success (Gardner \& Gardner 1969; Terrace et al. 1979), researchers nowadays primarily use a variety of abstract symbols (so-called lexigramms) where the ape learns that a certain symbol refers to a certain object or action (e.g. Premack 1971; Savage-Rumbaugh et al. 1993). The most famous languagetrained ape is probably Kanzi, the bonobo. Asked to carry out specific instructions in spoken English his understanding resembled that of 2-year-old children. Although, many of these studies face the problem of unintentional cueing by the experimenter and involve extensive training, they nonetheless indicate that apes can learn to understand human words and small sentences (see also Shettleworth 2010, p.541).

Consequently, we cannot generalize from the results of our experiments to cognitive abilities in other domains. Differences between monkeys and apes certainly exist, for example in selfrecognition or learning skills. But as Amici and colleagues also concluded (2010, p. 8): "[...] each cognitive skill represents a facet of intelligence that cannot be easily reduced to other ones. Monkeys and apes cannot be neatly classified into two homogeneous groups for every task." These results thus question the concept of cognition as a domain-general ability and the separation of species in terms of a general intelligence factor ( $g$ factor, Reader et al. 2011). Interestingly, this model is typically applied in human intelligence tests, which may also be unsuited to reveal individual differences (in fact, other approaches on "multiple intelligences" in humans do exist, Gardner 1983; Kincheloe 2007). Characterizing cognition as a set of specialpurpose abilities, which evolved in response to specific environmental challenges, seems therefore more appropriate.

Correspondingly, in our experiments we found differences within the two taxa (i.e. Hominoidea and Cercopithecoidae), which were rather influenced by ecological factors, such as diet, than by phylogenetic relatedness or brain size (Chapter 4). Gorillas had more difficulties in discriminating between small size differences of three-dimensional objects than did other apes 
and even monkeys. Gorillas are a primarily folivourous species, eating mostly leaves and foliage. In contrast, the other species, including both apes and monkeys, rely heavily on fruits. The differences in size discrimination abilities may have therefore evolved in response to differences in the species' diet, as being able to choose the larger of two fruit items is advantageous when competing with conspecifics.

To understand the evolution of intelligence more specific hypotheses should be developed and tested. As primates represent such a diverse order broad assumptions based on phylogeny or brain size are not sufficient to explain the many differences and similarities between species. Apparently, in the study reported in Chapter 4 feeding ecology seemed to have a significant influence on the species size discrimination abilities. Other cognitive abilities may be explained by other socio-ecological and environmental factors. Amici and colleagues (2008) compared the level of inhibitory control of monkeys and apes in five different tasks (for example delay of gratification) and revealed that species living in systems with fission-fusion dynamics (chimpanzees, bonobos, orangutans, and spider monkeys) outperformed members of species that live in more stable groups (long-tailed macaques, gorillas and capuchin monkeys). Apparently, the level of social complexity predicted the inhibitory skills better than phylogenetic relatedness or ecological conditions (Amici et al. 2008). However, such analyses are sometimes also problematic. In fact, to classify orangutans as a species with high levels of fission-fusion is disputable. These apes live mostly solitary and meet relatively infrequently (Kappeler \& van Schaik 2002). Although some researchers refer to this social system as fission-fusion (van Schaik 1999) it is notably different from the groupings of chimpanzees, bonobos and spider monkeys (Geissmann 2003). Putting these species into the same category seems therefore a bit arbitrary and calls the authors' conclusions into question.

\subsection{The Question of Social or Physical Cognition?}

As mentioned in the Introduction, Tomasello and Call (1997) defined and separated the terms social and physical cognition in their book Primate Cognition. Since then, many researchers have adopted their definitions, conducted experiments and interpreted their results in terms of one of the two domains. In fact, the design of the PCTB (Chapter 2) is based on Tomasello's and Call's theoretical assumptions. And a number of studies indeed suggest that there might be some kind of difference between socio- and physico-cognitive mechanisms, at least in primates. Some brain areas, specifically the amygdala, medial temporal lobe, orbitofrontal cortex, and superior temporal gyrus (the so-called 'social brain'; Brothers 1990) seem to be especially tuned to social stimuli. In particular, the primate visual system seemed to 
have been influenced by social pressures (Barton 1998; Barton 2006). Barton (2006, p. 233) states: "Converging evidence from cognitive neuroscience and comparative studies suggests that primate visual specialization is linked to sociality" (but see Barton 2006 for also alternative explanations). Barrett (2011) further exemplifies that the visual system of mammals consists of two path ways: the magnocellular pathway, which is common to all mammals, and the parvocellular pathway, which only exists in primates. The parvocellular pathway conveys the perception of fine detail and colour and is linked to the amygdale (which is involved in the perception of emotion, Gallagher \& Chiba 1996). Interestingly, especially the number of neurons associated with the parvocellular pathway seems to be linked to primate group size (Barton 1998, 2006; Barrett 2011). Researchers therefore conclude that it evolved "to process particularly important details of dynamic social stimuli, like facial expressions, gaze direction and posture" (Barrett 2011 p. 34; Perrett et al. 1992). The detection of so-called mirror neurons also favours the hypotheses that brain evolution has been shaped by social pressures. These neurons respond when an individual both performs a specific action and when it sees another individual perform the same action (Gallese et al. 1996; Rizzolatti et al. 1996; Hari et al. 1998; Gallese 2005). Intriguingly, they do not fire when the observed agent is a machine (Gallese 2001). These studies suggest that, at least in the primate order, social feature may have played a significant role in the evolution of specific brain functions maybe leading to specifically enhanced social skills.

However, the assumption that physical and social abilities rely on separable cognitive mechanisms is also debatable. As stated by Herrmann and colleagues (2007, p. 1366): "[...] it is possible that what is distinctively human is not social-cultural cognition as a specialized domain, as we have hypothesized. Rather, what may be distinctive is the ability to understand unobserved causal forces in general, including (as a special case) the mental states of others as causes of behaviour." Thus, causal reasoning and not specifically social capacities may explain differences between species and the outstanding abilities of humans. As already mentioned in Chapter 3, Penn and colleagues (2008) further propose that in fact a domain-general ability of representing abstract relationships and reinterpreting them in a systematic, rule-governed way specially distinguishes humans from other animals (see so-called "relational reinterpretation" hypothesis).

The socio-cognitive tasks of the PCTB reported in Chapter 2 did not allow to distinguish whether the animals indeed used special socio-cognitive mechanisms or solved the tasks using basic physical principles. In the PCTB, the baboons chose the cup the experimenter intended to grasp significantly above chance, and the macaques learned to use a human pointing gesture 
when we modified the experimental setup in the pointing study. However, as discussed in Chapter 2 and shown in Chapter 5, the monkeys seemed not to apply social skills, such as understanding others intentions, but rather used spatial associations, such as the proximity between the experimenter's hand and the cup, to find the reward. Only examining the results in more detail allowed us to distinguish between these alternatives, highlighting the problem of interpreting observed behaviours (see Chapter 6.3.). Future studies should try to develop similar experiments, which examine whether an animal used socio- or physico-cognitive skills to solve the task. As Byrne \& Whiten (1997, p. 14) pointed out: „If the intelligence is merely biased towards social tasks, then we would expect greater efficiency in social versions of a logically identical problem than in non-social ones."

\subsection{Explaining Animal Behaviour}

As just mentioned, inferring underlying cognitive capacities from observed behaviours is not always straightforward. Boyd and Silk (2002) provided a useful example: Imagine a female macaque does not react to her screaming infant. Observing this behaviour may either mean that 1) she is unaware that screams indicate that her infant is in distress, 2) she is unable to recognize that the screams are from her own infant, or 3) she avoids taking action because doing so could have negative consequences (e.g. because it is a higher ranking subject that has caused the infant's distress). From simply observing the situation one cannot discriminate between the three alternatives, which is a similar problem in laboratory studies.

In this thesis I have attempted to explain the monkeys' behaviour by focusing on the underlying mechanisms and avoiding anthropomorphic explanations. As shown in Chapter 5 the long-tailed macaques learned to use the human pointing cue in the course of the experiment. Some researchers equate the successful utilization of human gestures with the comprehension of its communicative intention (Hare et al. 2002). Although the monkeys showed not such a spontaneous reaction to human pointing as most dogs do, did the macaques now understand that the experimenter wanted to show them the place of the reward? If we had not tested the monkeys further, we would not have been able to draw any conclusions on this assumption. However, the kind of cue used to point at the correct location (human, doll, stick) did not have an influence on the monkeys' performance and they only used the cue when it was close to the cup, suggesting that they did in fact not have a special understanding of its communicative intent. Instead, local enhancement appeared to account for much of the monkeys' performance. 
As mentioned in the Introduction, how to explain animals' behaviour is continuously discussed and constitutes one of the main problems in comparative psychology (Hampton 2009; Balter 2012). Although most researchers agree that it is important to consider the most parsimonious explanation, there is much debate on what that actually means. Applying parsimony is based on the assumption that simpler explanations are, all else being equal, generally more likely to be closer to the truth than are more complex ones (Barrett 2011). Concerning animal behaviour this assumption is also known as Morgan's Canon: "In no case is an animal activity to be interpreted in terms of higher psychological processes, if it can be fairly interpreted in terms of processes which stand lower in the scale of psychological evolution and development." (Morgan 1894, p. 53). The question of which explanations are simpler and which processes are "lower in scale" is, however, not so easily answered.

Critics of the behaviouristic account (i.e. all behaviour results from learned associations) propose the principle of evolutionary and cognitive parsimony (de Waal 2008; Barrett 2011): If two closely related species behave in the same way, then the underlying mental processes are probably the same as well. Furthermore, explaining a particularly complex behaviour through a "more" cognitive explanation relying on representational elements would assume fewer steps as a purely associative account, which would assume a long chain of individual associations occurring in a specific order. Considering primates, these assumptions suggest that apes and humans who behave similarly probably have the same cognitive substructure underlying these behaviours, thus supporting anthropomorphic explanations. This suggestion is also one of the main reasons why researchers often attribute advanced cognitive skills to apes and separate them from monkeys. However, the experiments of my thesis suggest that the cognitive split between apes and monkeys may not be that clear-cut. The following two reasons will further highlight why the previous suggestion may not be justified.

First, although humans and apes are closely related, the amount of time that has passed since humans spilt from chimpanzees and bonobos (roughly 6 million years), is plenty enough for both lineages to have developed species-specific adaptations (Premack 2007; Barrett 2011). Indeed, chimpanzees and bonobos exhibit relatively large behavioural differences (Herrmann et al. 2010; see also Rilling et al. 2011 for interesting differences in the bonobo and chimpanzee brain), and their common ancestor dates back only 2 million years (Perelman et al. 2011). Furthermore, quite a few substantive differences are apparent between apes and humans, including the emergence of language, culture, religion and so on, suggesting that humans developed cognitive capacities not possessed by the great apes (see section 6.5.). We still know too little about the functions of the various DNA components (e.g. the non-coding regions) to 


\section{Chapter 6-General Discussion}

properly understand what a $98 \%$ genetic similarity really means. Interestingly, the macaque genome also shares approximately $92-95 \%$ of its sequence with humans and $98 \%$ with chimpanzees (Rhesus Macaque Genome Sequencing and Analysis Consortium 2007). The pure percentage of similar gene sequences therefore seems not to provide such meaningful insights into the cognitive similarities between species (in fact, even fruit flies share nearly $60 \%$ of human genes, Adams et al. 2000). Furthermore, the human brain differs significantly from that of apes, and recent studies have shown that the enormous development of the human brain, which has primarily taken place only in the last few hundred thousand years, is actually still continuing (Evans et al. 2005; Mekel-Bobrov et al. 2005). Accordingly, just as species-specific behaviours can change rapidly in evolution, so too could the mechanisms which underlie behaviours (Barrett 2011).

Second, as Morgan (1894) already noted, although an anthropomorphic explanation may seem simpler than one based on associations, we cannot easily decide which is the correct explanation. This problem is illustrated by the following example: "It is also much simpler to explain the existence of the organic world through the assumption that God created it in six days, than as a result of the long, slow, convoluted process of evolution" (Barrett 2011, p. 16). Accordingly, although an associative account seems more complex, this does not mean that it cannot explain a lot of animal behaviour. Furthermore, pitting associations against anthropomorphic explanations is an incorrect dichotomy (Barrett 2011). Explaining animal behaviour only through learning stimulus-outcome relations or through human-like thought, denies animals their own cognitive adaptations (see also Barrett 2009). Complex behaviour can also be explained by other mechanisms. In her recent book Beyond the Brain, Louise Barrett provides a useful example. She described robotic studies, which revealed that seemingly complex behaviour (for example intentional actions, mating dances or mirror self-recognition), were only due to the interaction of the robots' constructional features and the environment (see also Holland 2003 for a review). The robots thereby only followed simple rules such as "if sensory information is received on the left, then turn right, and if sensory information is received on the right, turn left" (Barrett 2009, p. 98). Similar examples can be found in the animal kingdom. The jumping spider (genus Portia) shows dozens of flexible behaviours to hunt its prey (e.g. complex detours and 'deceptive mimicry'), which all seem cognitively demanding. However, as with the robots, detailed studies revealed that all of these behaviours are due to the spider following one simple behavioural rule: "Keep going if you see a horizontal, turn back if you don't" (see e.g. the studies by Stimson Wilcox and the review by Harland \& Jackson 2000, 
Barrett 2011). This is concisely summarized by Barrett (2011): "Complex behaviour does not necessarily require complex internal mechanisms" (p.55).

Thus, the main problem of anthropomorphic accounts of cognition is its aim to reveal human-like capacities in animals (see Penn 2011 for a critical discussion). Thereby, unique capacities, which species developed to cope with environmental challenges, are neglected. Some animals possess abilities that humans lack completely: the echolocation system in bats, the electricity-producing organs of some fish, the magnetoception in birds (Griffin 1958; Watanabe \& Takeda 1963; Wiltschko \& Wiltschko 2005), to name just a few. These specific adaptations make it likely that animals also developed cognitive mechanisms that differ from our own. In the framework of "anthropocentrism", as Shettleworth (2012) calls it, these aspects would not be under close examination and we would miss fascinating insights into animal behaviour. Thus, future studies should try to focus on the behaviour and explain how it works instead of searching for the human link.

This is also inevitable for comparative studies. As MacLean and colleagues (2011) stated comparing the cognitive abilities of phlylogenetically closely related species constitutes a valuable account for future research. Nonetheless, one always has to keep in mind that not all tests are equally applicable to every species. Therefore examining the underlying mechanisms, which determine the species performance in a given experiment, is very important. These additional insights help to shed light on whether similar performances of different species are indeed due to similar cognitive mechanisms or whether dissimilarities are only revealed when digging deeper into the problem. As Amanda Seed stated in the book Animal Thinking (Menzel \& Fischer 2011, p. 125): “In many cases, two processes can cause the same behaviour in one class of problems, but they would typically show different outcomes when circumstances are modified." Systematic experiments then have to be conducted to discriminate between different alternatives (as for example described in Chapter 3).

\subsection{Are Humans different?}

Interpreting human behaviour can be equally difficult as judging animal behaviour and may sometimes be even misleading. In fact, whether all our behaviour is determined through the sophisticated mental operations, which are implied in most instances, or whether our daily activities sometimes rely on much simpler mechanisms is an open question (see Shettleworth 2012 for discussion). For example, recent studies testing human adults in similar experiments as apes and monkeys revealed the same irrational behaviour across species. Like apes, human subjects have been shown to avoid using a tool that brings a reward close to a trap, even when 
it could not possibly fall in (Silva \& Silva 2006). Likewise, humans will sometimes choose to pull a string, which only contacts a reward, but is actually not connected to it (Silva et al. 2008). Furthermore, Wright and colleagues (2006; cited in Shettleworth 2012) tested the differences on serial position effects in memory between monkeys, pigeons and humans. As the items to be remembered had no meaning to the animals, the humans were similarly tested with kaleidoscope patterns. The experiments revealed similar memory patterns in humans and animals and demonstrate a useful approach how to compare human to nonhuman subjects. Future studies should pursue similar directions and actually test the performance of human adults in the same task as animals before drawing conclusions on whether the animals' behaviour is truly different than our own.

Nonetheless, humans have certainly developed cognitive capacities different from their closest living relatives, which we did not explicitly test in our experiments. As mentioned earlier, many researchers attempted to train apes to use and understand human language. Although they achieved some success, no subject ever reached human-like capacities. Children exceed the competences of other species with about 2 to 3 years. As Terrace and colleagues (1979) for example revealed, the vocabulary of sign-language using children and the number of words in their sentences increases significantly with age (in both deaf and hearing children), whereas the vocabulary of the studied ape was confined to 125 signs after years of training and did not exceed three different signs per utterance. In fact, nonhuman primate vocal communication is characterized by little voluntary control on the structure of the animals' calls, which is predominantly innate (Hammerschmidt \& Fischer 2008; see Fischer 2010 for a review). Changes in structure are largely due to developmental factors, such as increasing body size or hormonal influences (Fischer et al. 2004; Ey et al. 2007; Pfefferle et al. 2008). Some more variation is evident on the receiver side. Comprehending the calls of conspecifics is largely influenced by learning. A playback study on infant chacma baboons, for example, showed that their reaction to alarm barks develops with age (Fischer et al. 2000; see also Fichtel 2008 for a similar study in lemurs). Furthermore, listeners can learn to attribute meaning to calls or call combinations, which can lead to appropriate behavioural reactions in response to different alarm calls (e.g. vervet monkeys react differently in response to alarm calls given to snakes, leopards or eagles, Seyfarth et al. 1980). Thus, nonhuman primates do probably not intentionally call to inform others, but their conspecifics can use this "potential information" (Fischer 2011) to cope with their environment. Accordingly, human language is significantly different from nonhuman primate vocal communication, as the calls of animals lack symbolic meanings and the agreement on their referential content (Fischer 2010). 
Likewise, whether the previously mentioned responses of apes to their reflections in a mirror indeed reveal human-like self-awareness is controversial (Shettleworth 2010). In contrast to human children, for example, not all ape subjects demonstrated mirror-guided self-recognition and passed the mark test. Nonetheless, as these tests are often regarded as demonstrating selfawareness, a prerequisite to develop a theory of mind (ToM), the success of some apes supported the claim that great apes do indeed possess ToM (Anderson \& Gallup 2011; Tomasello et al. 2003a). However, as already emphasized in the Introduction, many ToM tests may be explained by simpler assumptions, as for example behaviour reading, and also the reactions to a mirror may not need an understanding of oneself. Many studies suggest that truly understanding the minds of others as being different from one's own, having own representations, thoughts and goals, is probably specifically human (Penn \& Povinelli 2007; Penn 2011).

Similarly, although some behavioural traditions of wild chimpanzees may represent rudimentary forms of culture (Whiten et al. 1999; Whiten et al. 2001), only humans seem to have developed the necessary skills to participate and exchange knowledge in cultural groups as demonstrated in the PCTB (Herrmann et al. 2007). Tomasello and colleagues (Tomasello 2009; Tomasello \& Herrmann 2010) further argue that what makes us human is our uniquely cooperative nature, promoted by advanced socio-cognitive capacities such as shared intentionality. As shown in the experiments described in this thesis, apes and monkeys understand physical relations (also in the form of mental representations as shown in Chapter 3) and are able to use the behaviour of others to find for example food, but they are both distinguished from humans in their lack of understanding and motivation to cooperate, including such abilities as social learning via imitation and intentional communication (as indicated in Chapter 2 \& 5). As Rakoczy (2008) summarizes: "What lies at the heart of uniquely human cognition, though, and what lays the foundation for uniquely human sociality, is the ability to enter into collective 'WE'-intentionality" (p. 105).

\subsection{Problems of Comparative Studies}

As just described, interpreting the underlying cognitive mechanisms of an observed behaviour is tremendously difficult, even in humans. Furthermore, although the studies of this thesis suggest that monkeys and apes possess more similar cognitive abilities than has been previously thought, methodological aspects can significantly influence the results (as shown in Chapter 3). Although such an extensive comparative approach is essential for understanding cognitive evolution, there are also some limitations that I would like to reemphasise. The most 
problematic, applying the same test to different species may in reality not test the same factors (Tinbergen 1951). This applies especially to the widely used human-ape comparisons, as children are tested by their own species, whereas apes are not. Although studies try to refute this influence (e.g. Itakura et al. 1999; Herrmann et al. 2007), it is nonetheless a critical methodological dissimilarity which will be difficult to avoid. However, in this thesis I focused my comparisons on nonhuman primate species, resulting in testing situations that were more or less the same for all individuals. Nonetheless, as the tool use experiment of the PCTB illustrates (Chapter 2), different nonhuman primate species show considerable differences in their day-today life, which influence the validity of experiments. The baboons and macaques did not use a stick to retrieve out of reach food, however, in contrast to the apes they are also not known to display such behaviour in the wild. Comparative tests, which are really the same for every species, are hard to develop, if not impossible, as every species has its unique adaptations. This applies especially to situations where tests yield different results for different species. In such instances, it is necessary to further investigate why a given species apparently fails in a certain experiment.

As shown in Chapter 3 and 5, examining specific paradigms more closely can reveal that methodological aspects play a significant role in the performance and thus in the comparison of different species. We could for instance show that the quantity discrimination abilities of the monkeys could be significantly enhanced when choice stimuli and reward were separate entities. In Chapter 5 we could moreover show that monkeys can learn to use a human pointing gesture very quickly, which has not been shown in other studies. Varying the setup can thus have a tremendous effect on performance and the subsequent interpretation of an animal's capacity.

One additional weakness of the PCTB is its imbalance between the levels of difficulty of the physico- and socio-cognitive tasks for nonhuman primates. As discussed in Chapter 2, the PCTB mainly comprises tests developed to examine the ontogeny of human specific skills (social learning, communication, theory of mind). It may therefore well be that especially the social tasks have been too difficult to find a measurable difference between monkeys and apes. Furthermore, the good performance in the physical domain may represent a ceiling effect, meaning that these experiments were structurally simpler and therefore resulted in better performances in many of the tasks. Future comparative studies should try to develop experiments which test social and physical abilities on an equal level of difficulty. In the course of my studies we conducted several additional experiments with the monkeys, which I will 
briefly describe now. These will provide some suggestions for modifications and extension of the test battery.

\subsection{Suggestions for Future Studies}

"Comparative psychologists will need to focus on standardizing the essential components of each task while allowing for variation in other parameters required for a valid comparison between species."

MacLean et al. 2011, p. 233

Throughout this discussion I already emphasized some areas of future research, which would provide valuable insights into animal and human behaviour. Now I would like to give more specific examples on how to improve and expand some experiments of the PCTB, shortly present new methodological approaches and stress the importance of field studies (see also Menzel \& Fischer 2011 for interesting and useful suggestions on future research on animal cognition).

\subsection{1. РCTB plus}

Although the PCTB provides a valuable paradigm to test the cognitive differences of nonhuman primates, as mentioned earlier the test battery has a number of limitations. First, I recommend that future studies should also conduct the controls we added to some of the tasks (see Chapter 2) to rule out alternative explanations. I will now give some suggestions for additional tests, which I think would improve the battery for future usage (see also the discussion in Chapter 2). Testing the basal capacities of various species, but extending the experiments in case of positive results, would for example help to control for ceiling effects masking possible species differences.

\subsubsection{Physical Domain}

\section{Reversal Learning}

In addition to the quantity discrimination experiments of the PCTB (where I would recommend to use pebbles instead of food), I would suggest to also test the reversal learning abilities of different species. As many studies have shown, nearly every animal species is capable to discriminate between different quantities, making this task probably too easy to reveal species differences. Learning to choose the smaller amount instead constitutes a much more demanding task and there are already a number of studies testing this ability in different animal species (although most of them used food to test for differences in inhibitory control, see Boysen \& Berntson 1995, or Shifferman 2009 for a review). In the course of the quantity discrimination experiments described in Chapter 3, I tested whether the baboons would also be able to learn the reversal task and choose the smaller instead of the larger amount. As in the 
previous tests, the baboons first passed a familiarization phase with two quantity combinations ( 7 vs. 1 and 8 vs. 2) represented by pebbles. The subjects then received a reward only for choosing the smaller amount. After reaching criterion (80\% correct in two consecutive sessions) the same quantity combinations as in the previous experiments were tested (see Chapter 3). Three of the four baboons learned to choose the smaller amount of pebbles relatively quickly ( 7 to 10 sessions), thus I would recommend to use pebbles, as using food items requires inhibitory control and leads to much longer learning phases. The baboons have shown that subjects are able to learn the reversed variant relatively quickly, which is very important when conducting a large battery of tasks.

\section{Causal Reasoning}

As mentioned in the discussion of Chapter 2, although the monkeys were able to solve most of the tasks in the physical domain, it is not clear whether they really had an understanding of the underlying physical properties. It may well be that one would find differences between species when analyzing more specifically how the subjects solved the different tasks (Call 2004). At the moment Christian Schloegl conducts a series of experiments on causal reasoning in the long-tailed macaques (Schloegl, Waldmann \& Fischer, in preparation), as the experiments of the PCTB suggested that the monkeys possess a quite good understanding of the causal relations between two objects. However, Schloegl's detailed experiments now revealed that the causal understanding of the monkeys may not be that sophisticated. He tested, whether the monkeys understand that food could only be hidden under one of three different objects (cup, flat board, inclined board). In each trial only two of these options were presented, for example the cup and a flat board, in which case the food had to be under the cup. In this more demanding setup the macaques performed at chance level. They were not able to take the physical properties of the objects into account to locate the reward. It would be relatively easy to add these experimental variations to the normal task of the PCTB. If subjects would succeed in choosing the inclined instead of the flat board in the initial setup, than one could test whether they could also discriminate between these additional options, which would perhaps better identify species differences.

\subsubsection{Social Domain}

As the socio-cognitive tasks of the PCTB where primarily designed to test human specific capabilities, most of them lack ecological relevance for nonhuman primates. Furthermore, whether they actually test social skills is disputable. As demonstrated in Chapter 5 examining the underlying mechanisms that help subjects to solve the task, would provide a valuable 
account for future studies. Moreover, we conducted an additional experiment on the baboons' social learning abilities, which I will describe in more detail now. This kind of experiment could also be conducted relatively easily in future studies and would allow fairer comparisons between species compared to the tube tasks of the PCTB.

\section{Social Learning}

Given the extraordinary social skills baboons exhibit in the wild, we decided to have a closer look at their social learning abilities, specifically in terms of imitation. Whether animals are able to imitate is highly debated (Voelkl \& Huber 2000; Stoinski et al. 2001; Stoinski \& Whiten 2003; Box \& Russon 2004; Call et al. 2005; Horner \& Whiten 2005; Custance et al. 2006), but social learning in baboons has to date not received much attention (see Beck 1973 for one observational study).

In the course of the PCTB, we tested whether the baboons would use the same means to extract food from three different tubes as a human demonstrator. None of the subjects showed reliable indications for imitation. Almeling (2009) therefore conducted an additional experiment (the "artificial fruit" task; Galef Jr et al. 1986; Bugnyar \& Huber 1997; Voelkl \& Huber 2000) with the olive baboons using a transparent box, which could either be opened by turning a lever or pushing a button. Working with two groups of baboons, she demonstrated lever-turning to one group and button-pushing to the other.

As in the PСTB experiments, none of the baboons used the method demonstrated by the experimenter. In contrast, they all tried to solve the task on their own and some also succeeded, not taking the demonstrated action into account. Thus, although we tried to implement an easier and more straightforward task to test social learning in baboons, they did not show any indication of benefiting from the demonstration, neither in the form of imitation nor in the form of other social learning mechanisms (e.g. emulation). Of course, absence of evidence is not evidence of absence. Thus, although we did not find evidence for social learning in baboons in our experiments, it does not mean that they are not able to use it (in fact there are studies suggesting that monkeys do learn socially, see Zuberbühler et al. 1996). The fact that we used human demonstrators instead of conspecifics (due to methodological constraints) may restrain the subjects from using the cues. Indeed, as we have shown in Chapter 5, the presence of the experimenters' body and face seemed to inhibit the monkeys from using a pointing cue. Perhaps this is also the case in other socio-cognitive tasks. Experiments on social learning in orangutans, in contrast, found no effect of human versus conspecific demonstrators (Call \& Tomasello 1995). Further tests have to be conducted to better understand the influence of humans in such kind of experiments. 


\section{Chapter 6-General Discussion}

Constructional features may also have prevented the baboons from learning from the demonstrations. In the tube tasks of the PCTB, a lack of inhibitory control clearly influenced the monkeys' responses. With the transparent tubes the subjects saw the reward and tried to gain access to it by biting the tube or reaching directly for the food. These constraint responses seemed to prevent the monkeys from using the demonstrated actions (e.g. banging the tube on the ground vertically). Furthermore, in the box task, pressing the button seemed to be not as straightforward as turning the lever. All baboons, which learned to open the box, did so by using the lever. This difficulty may in part explain their failure to use the demonstrated action.

Future studies should therefore try to minimize such confounding effects. A useful example is provided in a recent study by Gunhold and Bugnyar (2011) with wild marmosets. They presented the subjects with a container baited with food, which could be opened in two different ways (similar to our box used with the baboons). Then they showed different groups videos demonstrating one way to open the box, i.e. either pulling a drawer or pushing a lid (in another study they trained one conspecific to open the box). In the control groups no individual showed a preferred foraging technique. The individuals in the video groups, in contrast, tended to be more successful and predominantly used the technique demonstrated in the videos.

In conclusion, the tube tasks of the PCTB seem not to be appropriate to test the social learning abilities of nonhuman primates. Here the initial design for developmental studies in human children becomes too apparent, as the tasks show little ecological relevance for nonhuman primates. The two-action method we used to test the learning abilities of the baboons and the artificial-fruit task already used successfully in the wild (Gunhold \& Bugnyar 2011) would provide more appropriate designs (see also Schnoell \& Fichtel, in press). One could either train subjects to use one of the methods (by blocking the alternative possibility) or demonstrate only one technique to specific subjects and then see whether observing individuals would use the same action.

\subsubsection{Touch Screen Experiments}

Another valuable account to explore the cognitive capacities of different animals in more detail is the introduction of touch screen experiments, which are becoming increasingly common in cognitive research (Basile \& Hampton 2011; Hampton 2011; Truppa et al. 2011). This method allows the experimenter to control for confounding variables, such as the presence of a human experimenter, and provides the opportunity to use exactly the same setup for different species. Furthermore, it is very easy to change only specific details of a task and see which effect this has on the animal's performance. Specifically, this approach would allow to 
examine whether social or only physical aspect of a certain experiment determine species' performances. Recent studies for example test whether arrows and gaze cues lead to the same eye movements in human participants (Kuhn \& Kingston 2009). Another potentially fruitful avenue is the creation of artificial scenes, which could not be observed in nature. Hampton and colleagues (Paxton et al. 2010), for example, show rhesus monkeys videos of artificial social interactions of their conspecifics to test what the monkeys know about the relationships of others. Similar experiments have been conducted using playback experiments with baboons in the wild (see Cheney \& Seyfarth 2008). The touch screen setup now allows testing animals in standardized experiments. In many labs the animals can even access several touch screens throughout the day, leading to a generation of large data sets and allowing animals to be tested while they are within their social groups.

\subsubsection{Field Studies}

In addition to comparative experiments in the lab, which can complement field observations and test derived hypothesis in a controlled and systematic manner, comprehensive field observations are necessary to truly understand animal cognition. Bshary and colleagues (2011) also state: "[...] we think that research on animal cognition should have a much stronger field component, ideally conducting experiments in the field or on wild caught individuals, to complement standard laboratory experiments" (p. 220). As illustrated in the Introduction and shown in Chapter 5 ecological factors are important in shaping the cognitive and perceptual capacities of a given species. It is therefore essential to understand the ecology, including foraging techniques, and social organisation of the study species. But field observations not only form the basis for explaining possible species differences, they also help to derive new hypotheses, which then can be tested in the laboratory or the field. For examples, guinea baboons, largely unstudied in the wild until the last couple of years, are now known to have a social system that differs from other baboon species, and display unusually friendly male-male relationships (Patzelt et al. 2011). Based on these observations, researchers have begun to conduct playback experiments, which suggest that the animals discriminate between group and non-group members (Maciej et al. in preparation), with group sizes of sometimes more than 300 individuals. Similar playback experiments have been conducted with gelada baboons in Ethiopia, which also live in such large groups, but show a different substructure and social behaviour (Bergman 2010; see also Bergman \& Kitchen 2009 for a comparative study in wild baboons and geladas). Differences in the responses to the playbacks can therefore be linked to differences in the species socio-ecology. 
Experimental field studies are also getting more and more sophisticated. As mentioned earlier studies have even been designed to show videos to free ranging animals (Gunhold \& Bugnyar, 2011); other studies have introduced artificial feeding stations to explore deceptive behaviour, spatial memory, decision-making and coordination in monkeys and lemurs (Janson 1998; Janson 2007; Wheeler 2009; Wheeler 2010; Pyritz 2011). These kinds of experiments are a promising research direction as they test animal behaviour in ecologically-relevant circumstances. Laboratory studies can complement such findings to get a detailed understanding of the underlying cognitive mechanisms.

\subsection{General Conclusions}

Studying the evolution of cognition can be accomplished in many different ways. In this thesis I focused on the socio- and physico-cognitive capacities of Old World monkeys to obtain a better understanding of primate cognitive evolution. The experiments described in this dissertation suggest that monkeys are not less intelligent than apes in the tests conducted. In contrast, in the tests of the PCTB, the apes' performance was more similar to that of monkeys than to humans. Our results reveal that monkeys understand physical relations and are able to use the behaviour of others to predict certain outcomes, but they do not seem to understand the intentional structure of communicative actions. Specific social factors therefore seem only relevant to distinguish humans from other apes, but not apes and monkeys. However, further tests have to be conducted as the design of the present experiments may have underestimated species differences. In fact, the additional examination of specific experiments highlighted the significant influence of methodological aspects. Moreover, these analyses provided insights into the factors determining the subjects' performances. A given species' ecology accounted for much more variation in cognitive abilities than did phylogenetic relatedness or brain size. In conclusion, as also emphasised by MacLean and colleagues (2011), comparative phylogenetic methods should be increasingly used in future research and interdisciplinary communication should be enhanced. 


\section{References}

Adams, M. D., Celniker, S. E., Holt, R. A., Evans, C. A., Gocayne, J. D., Amanatides, P. G., et al. 2000. The genome sequence of Drosophila melanogaster. Science, 287, 2185-2195.

Addessi, E., Crescimbene, L. \& Visalberghi, E. 2008a. Food and token quantity discrimination in capuchin monkeys (Cebus apella). Animal Cognition, 11, 275-282.

Addessi, E., Mancini, A., Crescimbene, L., Padoa-Schioppa, C. \& Visalberghi, E. 2008b. Preference transitivity and symbolic representation in capuchin monkeys (Cebus apella). PLOS ONE, 3, e2414.

Agrillo, C., Dadda, M. \& Bisazza, A. 2007. Quantity discrimination in female mosquitofish. Animal Cognition, 10, 63-70.

Agrillo, C., Dadda, M., Serena, G. \& Bisazza, A. 2008. Do fish count? Spontaneous discrimination of quantity in female mosquitofish. Animal Cognition, 11, 495-503.

Agrillo, C., Piffer, L., Bisazza, A. \& Butterworth, B. 2012. Evidence for two numerical systems that are similar in humans and guppies. PLOS ONE, 7, e31923.

Aiello, L. C. \& Wheeler, P. 1995. The expensive-tissue hypothesis: the brain and the digestive system in human and primate evolution. Current Anthropology, 36, 199-221.

Almeling, L. 2009. Social learning in olive baboons. Bachelorthesis, Georg-August University Göttingen.

Amici, F., Aureli, F. \& Call, J. 2008. Fission-fusion dynamics, behavioral flexibility, and inhibitory control in primates. Current Biology, 18, 1415-1419.

Amici, F., Aureli, F. \& Call, J. 2010. Monkeys and apes: Are their cognitive skills really so different? American Journal of Physical Anthropology, 143, 188-197.

Amici, F., Call, J. \& Aureli, F. 2009. Variation in withholding of information in three monkey species. Proceedings of the Royal Society of London Series B: Biological Sciences, 276, 3311-3318.

Anderson, J. R. \& Gallup, G. G. 2011. Which primates recognize themselves in mirrors? PLoS Biology, 9, e1001024.

Anderson, J. R., Montant, M. \& Schmitt, D. 1996. Rhesus monkeys fail to use gaze direction as an experimenter-given cue in an object-choice task. Behavioural Processes, 37, 47-55.

Anderson, U. S., Stoinski, T. S., Bloomsmith, M. A. \& Maple, T. L. 2007. Relative numerousness judgment and summation in young, middle-aged, and older adult Orangutans (Pongo pygmaeus abelii and Pongo pygmaeus pygmaeus). Journal of Comparative Psychology, 121, 1-11.

Anderson, U. S., Stoinski, T. S., Bloomsmith, M. A., Marr, M. J., Smith, A. D. \& Maple, T. L. 2005. Relative numerousness judgment and summation in young and old western lowland gorillas. Journal of Comparative Psychology, 119, 285-295.

Andersson, M. 1994. Sexual Selection. Princeton, NJ: Princeton University Press.

Baillargeon, R. \& DeVos, J. 1991. Object permanence in young infants: further evidence. Child Development, 62, 1227-1246.

Balter, M. 2012. 'Killjoys' challenge claims of clever animals. Science, 335, 1036-1037. 
Banerjee, K., Chabris, C. F., Johnson, V. E., Lee, J. J., Tsao, F. \& Hauser, M. D. 2009. General intelligence in another primate: individual differences across cognitive task performance in a New World monkey (Saguinus oedipus). PLOS ONE, 4, e5883.

Barrett, L. 2009. A guide to practical babooning: Historical, social, and cognitive contingency. Evolutionary Anthropology: Issues, News, and Reviews, 18, 91-102.

Barrett, L. 2011. Beyond the Brain: How Body and Environment Shape Animal and Human Minds. Princeton, NJ: Princeton University Press.

Barrett, L., Dunbar, R. I. M. \& Lycett, J. 2002. Human Evolutionary Psychology. Princeton, NJ: Princeton University Press.

Barrett, L., Henzi, P. \& Dunbar, R. 2003. Primate cognition: from 'what now?' to 'what if?'. Trends in Cognitive Sciences, 7, 494-497.

Barton, R. A. 1998. Visual specialization and brain evolution in primates. Proceedings of the Royal Society of London Series B: Biological Sciences, 265, 1933-1937.

Barton, R. A. 2006. Primate brain evolution: Integrating comparative, neuro-physiological, and ethological data. Evolutionary Anthropology: Issues, News, and Reviews, 15, 224-236.

Barton, R. A. 2007. Evolutionary specialization in mammalian cortical structure. Journal of Evolutionary Biology, 20, 1504-1511.

Basile, B. M. \& Hampton, R. R. 2011. Monkeys recall and reproduce simple shapes from memory. Current Biology, 21, 774-778.

Beck, B. B. 1973. Observation learning of tool use by captive guinea baboons (Papio papio). American Journal of Physical Anthropology, 38, 579-582.

Beck, B. B. 1982. Chimpocentrism: Bias in cognitive ethology. Journal of Human Evolution, 11, 317.

Behne, T., Carpenter, M. \& Tomasello, M. 2005. One-year-olds comprehend the communicative intentions behind gestures in a hiding game. Developmental Science, 8, 492-499.

Beran, M. J. 2007. Rhesus monkeys (Macaca mulatta) enumerate large and small sequentially presented sets of items using analog numerical representations. Journal of Experimental Psychology: Animal Behavior Processes, 33, 42-54.

Beran, M. J. 2008. Monkeys (Macaca mulatta and Cebus apella) track, enumerate, and compare multiple sets of moving items. Journal of Experimental Psychology: Animal Behavior Processes, 34, 63-74.

Beran, M. J., Beran, M. M., Harris, E. H. \& Washburn, D. A. 2005. Ordinal judgments and summation of nonvisible sets of food items by two chimpanzees and a rhesus macaque. Journal of Experimental Psychology: Animal Behavior Processes, 31, 351-362.

Beran, M. J., Evans, T. A. \& Harris, E. H. 2008. Perception of food amounts by chimpanzees based on the number, size, contour length and visibility of items. Animal Behaviour, 75, 1793-1802.

Bergman, T. \& Kitchen, D. 2009. Comparing responses to novel objects in wild baboons (Papio ursinus) and geladas (Theropithecus gelada). Animal Cognition, 12, 63-73.

Bergman, T. J. 2010. Experimental evidence for limited vocal recognition in a wild primate: implications for the social complexity hypothesis. Proceedings of the Royal Society of London Series B: Biological Sciences, 277, 3045-3053. 
Birch, H. G. 1945. The relation of previous experience to insightful problem-solving. Journal of Comparative Psychology, 38, 367-383.

Bird, C. D. \& Emery, N. J. 2009. Rooks use stones to raise the water level to reach a floating worm. Current Biology, 19, 1410-1414.

Bitterman, M. E. 1960. Toward a comparative psychology of learning. American Psychologist, 15, 704-712.

Bitterman, M. E. 1965. Phyletic differences in learning. American Psychologist, 20, 396-410.

Blaisdell, A. P., Sawa, K., Leising, K. J. \& Waldmann, M. R. 2006. Causal reasoning in rats. Science, 311, 1020-1022.

Boesch, C. 2007. What makes us human (Homo sapiens)? The challenge of cognitive crossspecies comparison. Journal of Comparative Psychology, 121, 227-240.

Box, H. O. \& Russon, A. E. 2004. Social mediated learning among monkeys and apes: Some comparative perspectives. In: Comparative Vertebrate Cognition: Are Primates Superior to Non-Primates (Ed. by L. J. Rogers \& G. Kaplan), pp. 97-140. New York: Kluwer/Academic Plenum.

Boyd, R. \& Silk, J. B. 2002. How humans evolved. New York: W.W. Norton \& Company.

Boysen, S. T. \& Berntson, G. G. 1995. Responses to quantity: Perceptual versus cognitive mechanisms in chimpanzees (Pan troglodytes). Journal of Experimental Psychology: Animal Behavior Processes, 21, 82-86.

Brannon, E. M. \& Terrace, H. S. 1998. Ordering of the numerosities 1 to 9 by monkeys. Science, $282,746$.

Brothers, L. 1990. The social brain: a project for integrating primate behaviour and neurophysiology in a new domain. Concepts in Neuroscience, 1, 27-51.

Bshary, R., Di Lascio, F., Pinto, A. \& Van de Waal, E. 2011. How intelligent is Machiavellian behavior? In: Animal Thinking: Contemporary Issues in Comparative Psychology (Ed. by R. Menzel \& J. Fischer), pp. 209-221. Cambridge: MIT Press.

Bugnyar, T. \& Huber, L. 1997. Push or pull: an experimental study on imitation in marmosets. Animal Behaviour, 54, 817-831.

Bugnyar, T. \& Kotrschal, K. 2004. Leading a conspecific away from food in ravens (Corvus corax)? Animal Cognition, 7, 69-76.

Burkart, J. M. \& Heschl, A. 2007. Understanding visual access in common marmosets, Callithrix jacchus: perspective taking or behaviour reading? Animal Behaviour, 73, 457-469.

Burkart, J. M. \& van Schaik, C. 2012. Group service in macaques (Macaca fuscata), capuchins (Cebus apella) and marmosets (Callithrix jacchus): A comparative approach to identifying proactive prosocial motivations. Journal of Comparative Psychology, doi: $10.1037 / \mathrm{a} 0026392$.

Byrne, R. W. 1995. Primate cogntion: comparing problems and skills. American Journal of Primatology, 37, 127-141.

Byrne, R. W. 1996. Relating brain size to intelligence. In: Modelling the early human mind (Ed. by P. A. Mellars \& K. R. Gibson), pp. 49-56. Cambridge: McDonald Institute for Archaelogical Research. 
Byrne, R. W. 1997. The technical intelligence hypothesis: an additional evolutionary stimulus to intelligence. In: Machiavellian Intelligence II (Ed. by A. Whiten \& R. W. Byrne), pp. 289311. Cambrige: Cambrige University Press.

Byrne, R. W. 2000. Evolution of primate cognition. Cognitive Science, 24, 543-570.

Byrne, R. W. \& Bates, L. A. 2010. Primate social cognition: uniquely primate, uniquely social, or just unique? Neuron, 65, 815-830.

Byrne, R. W. \& Corp, N. 2004. Neocortex size predicts deception rate in primates. Proceedings of the Royal Society of London Series B: Biological Sciences, 271, 1693-1699.

Byrne, R. W. \& Whiten, A. 1988. Machiavellian Intelligence. Oxford: Oxford University Press.

Byrne, R. W. \& Whiten, A. 1997. Machiavellian Intelligence II: Extensions and Evaluations. Cambridge: Cambridge University Press.

Call, J. 2000. Estimating and operating on discrete quantities in orangutans (Pongo pygmaeus). Journal of Comparative Psychology, 114, 136-147.

Call, J. 2004. Inferences about the location of food in the great apes (Pan paniscus, Pan troglodytes, Gorilla gorilla, and Pongo pygmaeus). Journal of Comparative Psychology, $118,232-241$.

Call, J. 2007. Apes know that hidden objects can affect the orientation of other objects. Cognition, 105, 1-25.

Call, J., Carpenter, M. \& Tomasello, M. 2005. Copying results and copying actions in the process of social learning: chimpanzees (Pan troglodytes) and human children (Homo sapiens). Animal Cognition, 8, 151-163.

Call, J., Hare, B., Carpenter, M. \& Tomasello, M. 2004. 'Unwilling' versus 'unable': chimpanzees' understanding of human intentional action. Developmental Science, 7, 488-498.

Call, J. \& Tomasello, M. 1994. Production and comprehesion of referential pointing by orangutans. Journal of Comparative Psychology, 108, 307-317.

Call, J. \& Tomasello, M. 1995. Use of social information in the problem solving of orangutans (Pongo pygmaeus) and human children (Homa sapiens). Journal of Comparative Psychology, 109, 308-320.

Cantlon, J. F. \& Brannon, E. M. 2007. Basic math in monkeys and college students. Plos Biology, 5, e328.

Carazo, P., Font, E., Forteza-Behrendt, E. \& Desfilis, E. 2009. Quantity discrimination in Tenebrio molitor: evidence of numerosity discrimination in an invertebrate? Animal Cognition, 12, 463-470.

Carlson, N. R. 2007. Physiology of Behaviour. 9th Pearson International Edition. Boston: Allyn \& Bacon.

Carlson, S. M., Davis, A. C. \& Leach, J. G. 2005. Less is more. Psychological Science, 16, 609-616.

Charlton, B. D., Reby, D. \& McComb, K. 2007. Female red deer prefer the roars of larger males. Biology Letters, 3, 382-385.

Cheney, D. L. \& Seyfarth, R. M. 1992. How Monkeys See the World: Inside the Mind of Another Species. Chicago: University of Chicago Press.

Cheney, D. L. \& Seyfarth, R. M. 2008. Baboon Metaphysics: The Evolution of a Social Mind. Chicago: University of Chicago Press. 
Chittka, L. \& Niven, J. 2009. Are bigger brains better? Current Biology, 19, R995-R1008.

Clayton, N. \& Emery, N. 2005. Corvid cognition. Current Biology, 15, R80-R81.

Cloarec, A. 1986. Distance and size discrimination in a water stick insect, Ranatra linearis (Heteroptera). Journal of Experimental Biology, 120, 59-77.

Custance, D., Prato Previde, E., Spiezo, C., Rigamonti, M. M. \& Poli, M. 2006. Social learning in pig-tailed macaques (Macaca nemestrina) and adults humans (Homo sapiens) on a twoaction artifical fruit. Journal of Comparative Psychology, 120, 303-313.

Darwin, C. 1871. The Decent of Man and Selection in Relation to Sex. London: John Murray.

De Waal, F. B. M. 1982. Chimpanzee Politics: Power and Sex among Apes. London: Jonathan Cape.

De Waal, F. B. M. 1999. Anthropomorphism and anthropodenial: Consistency in our thinking about humans and other animals. Philosophical Topics, 27, 255-280.

De Waal, F. B. M. 2005. A century of getting to know the chimpanzee. Nature, 437, 56-59.

De Waal, F. B. M. 2008. Putting the altruism back into altruism: the evolution of empathy. Annual Review of Psychology, 59, 279-300.

De Waal, F. B. M. 2009. Darwin's last laugh. Nature, 460, 175-175.

De Waal, F. B. M. \& Ferrari, P. F. 2012. The Primate Mind: Built to Connect with Other Minds. Cambridge: Harvard University Press.

Deacon, T. W. 1990. Rethinking mammalian brain evolution. American Zoologist, 30, 629-705.

Deaner, R. O., Isler, K., Burkart, J. \& van Schaik, C. 2007. Overall brain size, and not encephalization quotient, best predicts cognitive ability across non-human primates. Brain, Behavior and Evolution, 70, 115-124.

Deaner, R. O., Van Schaik, C. \& Johnson, V. 2006. Do some taxa have better domain-general cognition than others? A metaanalysis of nonhuman primate studies. Evolutionary Psychology, 4, 149-196.

Dehaene, S. 1997. The Number Sense. Oxford: Oxford University Press.

Dehaene, S., Dehaene-Lambertz, G. \& Cohen, L. 1998. Abstract representations of numbers in the animal and human brain. Trends in Neurosciences, 21, 355-361.

DeLoache, J. S. 2000. Dual representation and young children's use of scale models. Child Development, 71, 329.

Dennett, D. C. 1987. The Intentional Stance. Cambridge: MIT Press.

Deschner, T., Heistermann, M., Hodges, K. \& Boesch, C. 2004. Female sexual swelling size, timing of ovulation, and male behavior in wild West African chimpanzees. Hormones and Behavior, 46, 204-215.

Diester, I. \& Nieder, A. 2010. Numerical values leave a semantic imprint on associated signs in monkeys. Journal of Cognitive Neuroscience, 22, 174-183.

Dunbar, R. I. M. 1992. Neocortex size as a constraint on group size in primates. Journal of Human Evolution, 22, 469-493.

Dunbar, R. I. M. 1998. The social brain hypothesis. Evolutionary Anthropology: Issues, News, and Reviews, 6, 178-190.

Dunbar, R. I. M. 2003. Evolution of the social brain. Science, 302, 1160-1161. 
Dunbar, R. I. M. 2009. Brain and behaviour in primate evolution. In: Mind the Gap: Tracing the Origins of Human Universals (Ed. by P. M. Kappeler \& J. B. Silk), pp. 315-330, Berlin, Heidelberg: Springer Verlag.

Dunbar, R. I. M. \& Shultz, S. 2007. Evolution in the social brain. Science, 317, 1344-1347.

Dwyer, C. M. \& Kendrick, K. M. 2008. Sheep senses, social cognition and capacity for consciousness. In: The Welfare of Sheep (Ed. by C. M. Dwyer), pp. 135-157, Berlin: Springer Verlag.

Emery, N. J. 2000. The eyes have it: the neuroethology, function and evolution of social gaze. Neuroscience and Biobehavioral Reviews, 24 581-604.

Emery, N. J. \& Clayton, N. S. 2001. Effects of experience and social context on prospective caching strategies by scrub jays. Nature, 414, 443-446.

Emery, N. J. \& Clayton, N. S. 2004a. Comparing the complex cognitive abilities of birds and primates. In: Comparative Vertebrate Cognition: Are Primates Superior to Non-Primates (Ed. by L. J. Rogers \& G. Kaplan). New York: Plenum Publishers.

Emery, N. J. \& Clayton, N. S. 2004b. The mentality of crows: convergent evolution of intelligence in corvids and apes. Science, 306, 1903-1907.

Emery, N. J. \& Clayton, N. S. 2005. Evolution of the avian brain and intelligence. Current Biology, 15, R946-R950.

Emery, N. J. \& Clayton, N. S. 2009. Comparative social cognition. Annual Review of Psychology, $60,87-113$.

Engelhardt, A., Pfeifer, J.-B., Heistermann, M., Niemitz, C., van Hooff, J. A. R. A. M. \& Hodges, J. K. 2004. Assessment of female reproductive status by male longtailed macaques, Macaca fascicularis, under natural conditions. Animal Behaviour, 67, 915-924.

Evans, P. D., Gilbert, S. L., Mekel-Bobrov, N., Vallender, E. J., Anderson, J. R., Vaez-Azizi, L. M., Tishkoff, S. A., Hudson, R. R. \& Lahn, B. T. 2005. Microcephalin, a gene regulating brain size, continues to evolve adaptively in humans. Science, 309, 1717-1720.

Evans, T. A., Beran, M. J. \& Addessi, E. 2010. Can nonhuman primates use tokens to represent and sum quantities? Journal of Comparative Psychology, 125, 369-380.

Evans, T. A., Beran, M. J., Harris, E. H. \& Rice, D. F. 2009. Quantity judgments of sequentially presented food items by capuchin monkeys (Cebus apella). Animal Cognition, 12, 97105.

Ey, E., Pfefferle, D. \& Fischer, J. 2007. Do age- and sex-related variations reliably reflect body size in non-human primate vocalizations? A review. Primates, 48, 253-267.

Fagot, J. \& Paleressompoulle, D. 2009. Automatic testing of cognitive performance in baboons maintained in social groups. Behavior Research Methods, 41, 396-404.

Fagot, J. \& Thompson, R. K. R. 2011. Generalized relational matching by guinea baboons (Papio papio) in two-by-two-item analogy problems. Psychological Science, 22, 1304-1309.

Feigenson, L., Dehaene, S. \& Spelke, E. 2004. Core systems of number. Trends in Cognitive Sciences, 8, 307-314.

Fichtel, C. 2008. Ontogeny of conspecific and heterospecific alarm call recognition in wild Verreaux's sifakas (Propithecus verreauxi verreauxi). American Journal of Primatology, 70, 127-135. 
Fischer, J. 2010. On the linguistic abilities of nonhuman primates (and some other animal species). In: Homo Novus - A human without illusions (Ed. by U. J. Frey, C. Störmer \& K. P. Willführ). Berlin, Heidelberg: Springer Verlag.

Fischer, J. 2011. Where is the information in animal communication. In: Animal Thinking: Contemporary Issues in Comparative Cognition (Ed. by R. Menzel \& J. Fischer), pp. 151161. Cambridge: MIT Press.

Fischer, J., Cheney, D. L. \& Seyfarth, R. M. 2000. Development of infant baboons' responses to graded bark variants. Proceedings of the Royal Society of London Series B: Biological Sciences, 267, 2317-2321.

Fischer, J., Kitchen, D., Seyfarth, R. \& Cheney, D. 2004. Baboon loud calls advertise male quality: Acoustic features and their relation to rank, age, and exhaustion. Behavioral Ecology and Sociobiology, 56, 140-148.

Fitch, W. T., Huber, L. \& Bugnyar, T. 2010. Social cognition and the evolution of language: constructing cognitive phylogenies. Neuron, 65, 795-814.

Flemming, T. M. \& Kennedy, E. H. 2011. Chimpanzee (Pan troglodytes) relational matching: playing by their own (analogical) rules. Journal of Comparative Psychology, 125, 207215.

Frith, U. \& Frith, C. 2010. The social brain: allowing humans to boldly go where no other species has been. Philosophical Transactions of the Royal Society B: Biological Sciences, 365, 165-176.

Galef Jr, B. G., Manzig, L. A. \& Field, R. M. 1986. Imitation learning in budgerigars: Dawson and Foss (1965) revisited. Behavioural Processes, 13, 191-202.

Gallagher, M. \& Chiba, A. A. 1996. The amygdala and emotion. Current Opinion in Neurobiology, 6, 221-227.

Gallese, V. 2001. The 'shared manifold' hypothesis: From mirror neurons to empathy. Journal of Consciousness Studies, 8, 33-50.

Gallese, V. 2005. Embodied simulation: From neurons to phenomenal experience. Phenomenology and the Cognitive Sciences, 4, 23-48.

Gallese, V., Fadiga, L., Fogassi, L. \& Rizzolatti, G. 1996. Action recognition in the premotor cortex. Brain, 119, 593-609.

Gallup, G. G. 1970. Chimpanzees: Self-Recognition. Science, 167, 86-87.

Gallup, G. G. 1982. Self-awareness and the emergence of mind in primates. American Journal of Primatology, 2, 237-248.

Gallup, G. G., Anderson, J. \& Shilito, D. J. 2002. The mirror test. In: The Cognitive Animal (Ed. by M. Bekoff, C. Allen \& G. M. Burghardt). Cambridge: MIT Press.

Gardner, H. 1983. Frames of Mind: The theory of multiple intelligences. New York: Basic Books.

Gardner, R. A. \& Gardner, B. T. 1969. Teaching sign language to a chimpanzee. Science, 165, 664-672.

Gazzaniga, M. S., Ivry, R. \& Mangun, G. R. 2002. Cognitive Neuroscience: The Biology of the Mind, 2nd edn. New York: W.W. Norton and Company.

Geissmann, T. 2003. Vergleichende Primatologie. Berlin, Heidelberg: Springer Verlag. 
Griffin, D. R. 1958. Listening in the Dark: The Acoustic Orientation of Bats and Men. New Haven: Yale University Press.

Griffin, D. R. 1981. The Question of Animal Awareness: Evolutionary Continuity of Mental Experience, 2nd edn. New York: Rockefeller University Press.

Griffin, D. R. 1984. Animal Thinking. Cambridge: Harvard University Press.

Gumert, M. D., Kluck, M. \& Malaivijitnond, S. 2009. The physical characteristics and usage patterns of stone axe and pounding hammers used by long-tailed macaques in the Andaman Sea region of Thailand. American Journal of Primatology, 71, 594-608.

Gunhold, T. \& Bugnyar, T. 2011. Learning from a virtual demonstrator in wild common marmosets (Callithrix jacchus). Poster presented at The Association for the Study of Animal Behaviour Summer Conference 2011.

Hammerschmidt, K. \& Fischer, J. 2008. Constraints in primate vocal production. In: The evolution of communicative creativity: from fixed signal to contextual flexibility (Ed. by U. Griebel \& K. Oller), pp. 93-119. Cambridge: MIT Press.

Hampton, R. R. 2009. Mulitple demonstrations of metacognition in nonhumans: converging evidence or multiple mechanisms? Comparative Cognition \& Behavior Reviews, 4, 17-28.

Hampton, R. R. 2011. Status of nonhuman memory monitoring and possible roles in planning and decision making. In: Animal Thinking: Contemporary Issues in Comparative Cognition (Ed. by R. Menzel \& J. Fischer), pp. 105-119. Cambridge: MIT Press.

Hare, B. 2007. From nonhuman to human mind: what changes and why. Current Directions in Psychological Science, 16, 60-64.

Hare, B., Addessi, E., Call, J., Tomasello, M. \& Visalberghi, E. 2003. Do capuchin monkeys, Cebus apella, know what conspecifics do and do not see? Animal Behaviour, 65, 131-142.

Hare, B., Brown, M., Williamson, C. \& Tomasello, M. 2002. The domestication of social cognition in dogs. Science, 298, 1634-1636.

Hare, B., Call, J., Agnetta, B. \& Tomasello, M. 2000. Chimpanzees know what conspecifics do and do not see. Animal Behaviour, 59, 771-785.

Hare, B., Call, J. \& Tomasello, M. 2001. Do chimpanzees know what conspecifics know? Animal Behaviour, 61, 139-151.

Hare, B. \& Tomasello, M. 2004. Chimpanzees are more skilful in competitive than in cooperative cognitive tasks. Animal Behaviour, 68, 571-581.

Hari, R., Forss, N., Avikainen, S., Kirveskari, E., Salenius, S. \& Rizzolatti, G. 1998. Activation of human primary motor cortex during action observation: A neuromagnetic study. Proceedings of the National Academy of Sciences, 95, 15061-15065.

Harland, D. P. \& Jackson, R. R. 2000. Cues by which Portia fimbriata, an araneophagic jumping spider, distinguishes jumping-spider prey from other prey. Journal of Experimental Biology, 203, 3485-3494.

Harlow, H. F. 1953. Mice, monkeys, men, and motives. Psychological Review, 60, 23-32.

Harlow, H. F. 1958. The evolution of learning. In: Behavior and Evolution (Ed. by A. Roe \& G. G. Simpson). New Haven, CT: Yale University Press.

Harlow, H. F., Harlow, M. K. \& Meyer, D. R. 1950. Learning motivates by a manipulative drive. Journal of Experimental Psychology, 40, 228-234. 
Harvey, P. H., Clutton-Brock, T. H. \& Mace, G. M. 1980. Brain size and ecology in small mammals and primates. Proceedings of the National Academy of Sciences of the United States of America, 77, 4387-4389.

Hauf, P. V. 2008. Two-dimensional psychophysics in chickens and humans: Comparative aspects of perceptual relativity. Japanese Psychological Research, 50, 167-182.

Haun, D. B. M., Jordan, F. M., Vallortigara, G. \& Clayton, N. S. 2010. Origins of spatial, temporal and numerical cognition: Insights from comparative psychology. Trends in Cognitive Sciences, 14, 552-560.

Hauser, M. \& Spelke, E. S. 2004. Evolutionary and developmental foundations of human knowledge: a case study of mathematics. In: The Cognitive Neuroscience (Ed. by M. Gazzaniga), pp. 853-864. Cambridge: MIT Press.

Hauser, M. D. 1996. The Evolution of Communication. Cambridge: MIT Press.

Hauser, M. D. 1997. Artifactual kinds and functional design features: What a primate understands without language. Cognition, 64, 285-308.

Hauser, M. D., Carey, S. \& Hauser, L. 2000. Spontaneous number representation in semi-freeranging rhesus monkeys. Proceedings of the Royal Society of London Series B: Biological Sciences, 267, 829-833.

Hauser, M. D., Kralik, J. \& Botto-mahan, C. 1999. Problem solving and functional design features: Experiments on cotton-top tamarins, Saguinus oedipus oedipus. Animal Behaviour, 57, 565-582.

Healy, S. D. \& Rowe, C. 2007. A critique of comparative studies of brain size. Proceedings of the Royal Society of London Series B: Biological Sciences, 274, 453-464.

Herrmann, E., Call, J., Hernandez-Lloreda, M. V., Hare, B. \& Tomasello, M. 2007. Humans have evolved specialized skills of social cognition: The cultural intelligence hypothesis. Science, 317, 1360-1366.

Herrmann, E., Hare, B., Call, J. \& Tomasello, M. 2010. Differences in the cognitive skills of bonobos and chimpanzees. PLOS ONE, 5, e12438.

Heyes, C. M. 1994. Reflections on self-recognition in primates. Animal Behaviour, 47, 909-919.

Hille, P.-C. C. G. G. 2001. Haptic discrimination of size and texture in squirrel monkeys (Saimiri sciureus). Somatosensory \& Motor Research, 18, 50-61.

Holland, O. 2003. The first biologically inspired robots. Robotica, 21, 351-363.

Horner, V. \& Whiten, A. 2005. Causal knowledge and imitation/emulation switching in chimpanzees (Pan troglodytes) and children (Homa sapiens). Animal Cognition, 8, 164181.

Humphrey, N. K. 1976. The social function of intellect. In: Growing Points in Ethology (Ed. by P. P. G. Bateson \& R. A. Hinde), pp. 303-317. New York: Cambridge University Press.

Inoue-Nakamura, N. 1997. Mirror self-recognition in nonhuman primates: a phylogenetic approach. Japanese Psychological Research, 39, 266-275.

Isler, K. \& van Schaik, C. P. 2006. Metabolic costs of brain size evolution. Biology Letters, 2, 557560.

Itakura, S., Agnetta, B., Hare, B. \& Tomasello, M. 1999. Chimpanzee use of human and conspecific social cues to locate hidden food. Developmental Science, 2, 448-456. 
Janson, C. 1998. Experimental evidence for spatial memory in foraging wild capuchin monkeys, Cebus apella. Animal Behaviour, 55, 1229-1243.

Janson, C. 2007. Experimental evidence for route integration and strategic planning in wild capuchin monkeys. Animal Cognition, 10, 341-356.

Jerison, H. J. 1973. Evolution of the Brain and Intelligence. New York: Academic Press.

Jolly, A. 1966. Lemur Behavior. Chicago: Chicago University Press.

Jolly, A. 1972. The Evolution of Primate Behaviour. New York: Macmillan.

Kahrimanovic, M., Bergmann Tiest, W. \& Kappers, A. 2011. Discrimination thresholds for haptic perception of volume, surface area, and weight. Attention, Perception, \& Psychophysics, 73, 2649-2656.

Kaminski, J., Call, J. \& Fischer, J. 2004. Word learning in a domestic dog: Evidence for 'fast mapping'. Science, 304, 1682-1683.

Kappeler, P. \& van Schaik, C. 2004. Sexual Selection in Primates. Cambridge: Cambridge University Press.

Kappeler, P. M. \& Silk, J. B. 2010. Mind the Gap: Tracing the Origins of Human Universals. Berlin, Heidelberg: Springer Verlag.

Kappeler, P. M. \& Van Schaik, C. 2002. Sexual Selection in Primates: New and Comparative Perspectives. Cambridge: Cambridge University Press.

Karmiloff-Smith, A. 1992. Beyond Modularity: A developmental perspective on cognitive science: Cambridge: MIT Press.

Kennedy, E. H. \& Fragaszy, D. M. 2008. Analogical reasoning in a capuchin monkey (Cebus apella). Journal of Comparative Psychology, 122, 167-175.

Kincheloe, J. L. 2007. Multiple Intelligences Reconsidered. New York: Peter Lang.

Köhler, W. 1925. The Mentality of Apes. London, New York: K. Paul, Trench, Trubner \& Co.

Kudo, H. \& Dunbar, R. I. M. 2001. Neocortex size and social network size in primates. Animal Behaviour, 62, 711-722.

Kuhn, G. \& Kingston, A. 2009. Look away! Eyes and arrows engage oculomotor responses automatically. Attention, Perception, \& Psychophysics, 71, 314-327.

Lakatos, G., Soproni, K., Dóka, A. \& Miklósi, Á. 2009. A comparative approach to dogs' (Canis familiaris) and human infants' comprehension of various forms of pointing gestures. Animal Cognition, 12, 621-631.

Lewontin, R. C. 1998. The evolution of cognition: Questions we will never answer. In: An invitation to cognitive science, Volume 4: Methods, models, and conceptual issues (Ed. by D. Scarborough \& S. Sternberg). Cambridge: MIT Press.

MacLean, E., Matthews, L., Hare, B., Nunn, C., Anderson, R., Aureli, F., Brannon, E., Call, J., Drea, C., Emery, N., Haun, D., Herrmann, E., Jacobs, L., Platt, M., Rosati, A., Sandel, A., Schroepfer, K., Seed, A., Tan, J., van Schaik, C. \& Wobber, V. 2011. How does cognition evolve? Phylogenetic comparative psychology. Animal Cognition, 15, 223-238.

Malaivijitnond, S., Hamada, Y., Suryobroto, B. \& Takenaka, O. 2007a. Female long-tailed macaques with scrotum-like structure. American Journal of Primatology, 69, 721-735. 
Malaivijitnond, S., Lekprayoon, C., Tandavanittj, N., Panha, S., Cheewatham, C. \& Hamada, Y. 2007b. Stone-tool usage by Thai long-tailed macaques (Macaca fascicularis). American Journal of Primatology, 69, 227-233.

Marino, L., Connor, R. C., Fordyce, R. E., Herman, L. M., Hof, P. R., Lefebvre, L., Lusseau, D., McCowan, B., Nimchinsky, E. A., Pack, A. A., Rendell, L., Reidenberg, J. S., Reiss, D., Uhen, M. D., Van der Gucht, E. \& Whitehead, H. 2007. Cetaceans have complex brains for complex cognition. PLoS Biology, 5, e139.

Matsuno, T. \& Fujita, K. 2009. A comparative psychophysical approach to visual perception in primates. Primates, 50, 121-130.

Matsuzawa, T. 2001. Primate Origins of Human Cognition and Behavior. Hong Kong: Springer.

Matsuzawa, T. 2003. The Ai project: historical and ecological contexts. Animal Cognition, 6, 199211.

Mekel-Bobrov, N., Gilbert, S. L., Evans, P. D., Vallender, E. J., Anderson, J. R., Hudson, R. R., Tishkoff, S. A. \& Lahn, B. T. 2005. Ongoing adaptive evolution of ASPM, a brain size determinant in Homo sapiens. Science, 309, 1720-1722.

Menzel, R. \& Fischer, J. 2011. Animal Thinking: Contemporary Issues in Comparative Cognition (Strüngmann Forum Reports). Cambridge: MIT Press.

Miklósi, Á. 2002. Can dancing replace scientific approach: Lost (again) in chimpocentrism. Behavioral and Brain Sciences, 25, 633-634.

Miklósi, Á. \& Soproni, K. 2006. A comparative analysis of animals' understanding of the human pointing gesture. Animal Cognition, 9, 81-93.

Milton, K. 1988. Foraging behavior and the evolution of primate cognition. In: Machiavellian Intelligence (Ed. by A. Whiten \& R. W. Byrne), pp. 285-305. Oxford: Oxford University Press.

Mishkin, M. \& Hall, M. 1955. Discrimination along a size continuum following ablation of the inferior temporal convexity in monkeys. Journal of Comparative and Physiological Psychology, 48, 97-101.

Montgomery, S., Capellini, I., Barton, R. \& Mundy, N. 2010. Reconstructing the ups and downs of primate brain evolution: Implications for adaptive hypotheses and Homo floresiensis. BMC Biology, 8: 9.

Montgomery, S. H., Capellini, I., Venditti, C., Barton, R. A. \& Mundy, N. I. 2011. Adaptive evolution of four microcephaly genes and the evolution of brain size in anthropoid primates. Molecular Biology and Evolution, 28, 625-638.

Morgan, C. L. 1894. An Introduction to Comparative Psychology. London: W. Scott.

Morissette, P., Ricard, M. \& Décarie, T. G. 1995. Joint visual attention and pointing in infancy: A longitudinal study of comprehension. British Journal of Developmental Psychology, 13, 163-175.

Mulcahy, N. J. \& Hedge, V. 2012. Are great apes tested with an abject object-choice task? Animal Behaviour, 83, 313-321.

Nieder, A. 2005. Counting on neurons: The neurobiology of numerical competence. Nature Reviews Neuroscience, 6, 177-190.

Nieder, A., Diester, I. \& Tudusciuc, O. 2006. Temporal and spatial enumeration processes in the primate parietal cortex. Science, 313, 1431-1435. 
Nieder, A., Freedman, D. J. \& Miller, E. K. 2002. Representation of the quantity of visual items in the primate prefrontal cortex. Science, 297, 1708-1711.

Nieder, A. \& Miller, E. K. 2004. Analog numerical representations in rhesus monkeys: Evidence for parallel processing. Journal of Cognitive Neuroscience, 16, 889-901.

Nunn, C. 2011. The Comparative Approach in Evolutionary Anthroplogy and Biology. Chicago: University of Chicago Press.

Olthof, A., Iden, C. M. \& Roberts, W. A. 1997. Judgments of ordinality and summation of number symbols by squirrel monkeys (Saimiri sciureus). Journal of Experimental Psychology: Animal Behavior Processes, 23, 325-339.

Osorio, D. \& Vorobyev, M. 1996. Colour vision as an adaptation to frugivory in primates. Proceedings of the Royal Society of London Series B: Biological Sciences, 263, 593-599.

Ostner, J. 2006. Macaques. Current Biology, 16, R569-R570.

Pack, A. A. 2010. The synergy of laboratory and field studies of dolphin behavior and cognition. International Journal of Comparative Psychology, 23, 538-565.

Pack, A. A. \& Herman, L. M. 2004. Bottlenosed dolphins (Tursiops truncatus) comprehend the referent of both static and dynamic human gazing and pointing in an object-choice task. Journal of Comparative Psychology, 118, 160-171.

Pagel, M. \& Harvey, P. 1989. Taxonomic differences in the scaling of brain on body weight among mammals. Science, 244, 1589-1593.

Parker, S. T. \& Gibson, K. R. 1977. Object manipulation, tool use and sensorimotor intelligence as feeding adaptations in cebus monkeys and great apes. Journal of Human Evolution, 6 , 623-641.

Parker, S. T. \& Gibson, K. R. 1979. A developmental model for the evolution of language and intelligence in early hominids. Behavioral and Brain Sciences, 2, 367-381.

Parker, S. T., Mitchell, R. W. \& Miles, H. L. 1999. The Mentalities of Gorillas and Orangutans: Comparative perspectives. Cambridge: Cambridge University Press.

Passingham, R. E. 1981. Primate specializations in brain and intelligence. Symposium of the Zoological Society of London, 46, 361-388.

Patzelt, A., Zinner, D., Fickenscher, G., Diedhiou, S., Camara, B., Stahl, D. \& Fischer, J. 2011. Group composition of guinea baboons (Papio papio) at a water place suggests a fluid social organization. International Journal of Primatology, 32, 652-668.

Paxton, R., Basile, B. B., Adachi, I., Suzuki, W. A., Wilson, M. E. \& Hampton, R.R. 2010. Rhesus monkeys (Macaca mulatta) rapidly learn to select dominant individuals in videos of artificial social interactions between unfamiliar conspecifics. Journal of Comparative Psychology, 124, 395-401.

Penn, D. C. 2011. How folk psychology ruined comparative psychology: and how scrub jays can save it. In: Animal Thinking: Contemporary Issues in Comparative Cognition (Ed. by R. Menzel \& J. Fischer), pp. 253-265. Cambridge: MIT Press.

Penn, D. C., Holyoak, K. J. \& Povinelli, D. J. 2008. Darwin's mistake: Explaining the discontinuity between human and nonhuman minds. Behavioral and Brain Sciences, 31, 109-130.

Penn, D. C. \& Povinelli, D. J. 2007. Causal cognition in human and nonhuman animals: A comparative, critical review. Annual Review of Psychology, 58, 97-118. 
Pepperberg, I. M. 1990. An investigation into the cognitive capacities of an African Grey Parrot (Psittacus erithacus). In: Advances in the Study of Behavior (Ed. by P. J. B. Slater, J. S. Rosenblatt \& C. Beer). San Diego: Academic Press.

Pepperberg, I. M. 2004. Cognitive and communicative capacities of grey parrots implications for the enrichment of many species. Animal Welfare, 13, 203-208.

Pepperberg, I. M. 2006. Grey parrot numerical competence: a review. Animal Cognition, 9, 377391.

Pepperberg, I. M. \& Gordon, J. D. 2005. Number comprehension by a grey parrot (Psittacus erithacus), including a zero-like concept. Journal of Comparative Psychology, 119, 197209.

Perelman, P., Johnson, W. E., Roos, C., Seuánez, H. N., Horvath, J. E., Moreira, M. A. M., Kessing, B., Pontius, J., Roelke, M., Rumpler, Y., Schneider, M. P. C., Silva, A., O'Brien, S. J. \& Pecon-Slattery, J. 2011. A molecular phylogeny of living primates. PLoS Genetics, 7, e1001342.

Perrett, D. I., Hietanen, J. K., Oram, M. W., Benson, P. J. \& Rolls, E. T. 1992. Organization and functions of cells responsive to faces in the temporal cortex [and discussion]. Philosophical Transactions of the Royal Society of London. Series B: Biological Sciences, 335, 23-30.

Pfefferle, D., Brauch, K., Heistermann, M., Hodges, J. K. \& Fischer, J. 2008. Female Barbary macaque (Macaca sylvanus) copulation calls do not reveal the fertile phase but influence mating outcome. Proceedings of the Royal Society of London Series B: Biological Sciences, 275, 571-578.

Pinker, S. 2010. The cognitive niche: Coevolution of intelligence, sociality, and language. Proceedings of the National Academy of Sciences, 107, 8993-8999.

Pisa, P. \& Agrillo, C. 2009. Quantity discrimination in felines: a preliminary investigation of the domestic cat (Felis silvestris catus). Journal of Ethology, 27, 289-293.

Pitt, D. 2008. Mental Representation. In: The Stanford Encyclopedia of Philosophy (Ed. by E. N. Zalta): http://plato.stanford.edu/archives/fall2008/entries/mental-representation/.

Povinelli, D. J. 1993. Reconstructing the evolution of mind. American Psychologist, 48, 493-509.

Povinelli, D. J. \& Vonk, J. 2003. Chimpanzee minds: suspiciously human? Trends in Cognitive Sciences, 7, 157-160.

Povinelli, D. J. \& Vonk, J. 2004. We don't need a microscope to explore the chimpanzee's mind. Mind and Language, 19, 1-28.

Premack, D. 1971. Language in chimpanzee? Science, 172, 808-822.

Premack, D. 2007. Human and animal cognition: Continuity and discontinuity. Proceedings of the National Academy of Sciences, 104, 13861-13867.

Premack, D. \& Woodruff, G. 1978. Does the chimpanzee have a theory of mind? Behavioral and Brain Sciences, 1, 515-526.

Proops, L., McComb, K. \& Reby, D. 2009. Cross-modal individual recognition in domestic horses (Equus caballus). Proceedings of the National Academy of Sciences, 106, 947-951.

Pyritz, L. 2011. Determinants and outcomes of group coordination and decision-making in redfronted lemurs (Eulemur rufifrons). Dissertation, Georg-August University Göttingen. 
Raby, C. R., Alexis, D. M., Dickinson, A. \& Clayton, N. S. 2007. Planning for the future by western scrub-jays. Nature, 445, 919-921.

Rakoczy, H. 2008. Collective intentionality and uniquely human cognition. In: Learning from Animals? Examining the Nature of Human Uniqueness (Ed. by E. Neumann-Held \& L. Röska-Hardy), pp. 105-121. London: Psychology Press.

Rakoczy, H. 2009. Kinds of selves: A comparative view on the development of intentionality and self-consciousness. In: Social Roots of Self-Consciousness: Psychological and Philosophical Contributions (Ed. by W. Mack \& G. Reuter), pp. 13-33. Berlin: Akademie Verlag.

Rakoczy, H. 2010. From thought to language to thought: Towards a dialectical picture of the development of thinking and speaking. Grazer Philosophische Studien, 81, 79-105.

Rakoczy, H. \& Tomasello, M. 2007. The ontogeny of social ontology: Steps to shared intentionality and status functions. In: Intentional Acts and Institutional Facts: Essays on John Searle's Social Ontology (Ed. by S. L. Tsohatzidis), pp. 113-137. Berlin: Springer Verlag.

Reader, S. M., Hager, Y. \& Laland, K. N. 2011. The evolution of primate general and cultural intelligence. Philosophical Transactions of the Royal Society B: Biological Sciences, 366, 1017-1027.

Reader, S. M. \& Laland, K. N. 2002. Social intelligence, innovation, and enhanced brain size in primates. Proceedings of the National Academy of Sciences of the United States of America, 99, 4436-4441.

Reid, P. J. 2009. Adapting to the human world: Dogs' responsiveness to our social cues. Behavioural Processes, 80, 325-333.

Rhesus Macaque Genome Sequencing and Analysis Consortium 2007. Evolutionary and Biomedical Insights from the Rhesus Macaque Genome. Science, 316, 222-234.

Richards, R. J. 1987. Darwin and the emergence of evolutionary therories of mind and behavior. Chicago: University of Chicago Press.

Richerson, P. J. \& Boyd, R. 1998. The evolution of human ultra-sociality. In: Ideology, Warfare, and Indoctrinability (Ed. by I. Eibl-Eibisfeldt \& F. Salter), pp. 71-96. New York: Berghan Books.

Rilling, J. K., Scholz, J., Preuss, T. M., Glasser, M. F., Errangi, B. K. \& Behrens, T. E. 2011. Differences between chimpanzees and bonobos in neural systems supporting social cognition. Social Cognitive and Affective Neuroscience, 7, 369-379.

Rizzolatti, G., Fadiga, L., Gallese, V. \& Fogassi, L. 1996. Premotor cortex and the recognition of motor actions. Cognitive Brain Research, 3, 131-141.

Rogers, L. J. 2004. Increasing the brain's capacity: Neocortex, new neurons, and hemispheric specialization. In: Comparative Vertebrate Cognition: Are Primates Superior to NonPrimates (Ed. by L. J. Rogers \& G. Kaplan), pp. 289-324. New York: Kluwer Academic/Plenum Publishers.

Rogers, L. J. \& Kaplan, G. 2004. Comparative Vertebrate Cognition: Are Primates Superior to Non-Primates? New York, Boston, Dordrecht, London, Moscow: Kluwer Academic/Plenum Publishers.

Rugani, R., Regolin, L. \& Vallortigara, G. 2008. Discrimination of small numerosities in young chicks. Journal of Experimental Psychology: Animal Behavior Processes, 34, 388-399. 
Russon, A. E. \& Begun, D. R. 2004. The Evolution of Thought: Evolutionary Origins of Great Ape Intelligence. Cambridge: Cambridge University Press.

Sandel, A. A., MacLean, E. L. \& Hare, B. 2011. Evidence from four lemur species that ringtailed lemur social cognition converges with that of haplorhine primates. Animal Behaviour, 81, 925-931.

Sarris, V., Hauf, P. \& Arlt, M. 2001. The animal psychophysics of "relative" stimulus discrimination: color and size data from the baby chick. In: Fechner Day 2001. International Society for Psychophysics, 17th Annual Meeting (Ed. by E. Sommerfeld, R. Kompass \& T. Lachmann), pp. 576-581. Lengerich: Pabst.

Savage-Rumbaugh, E. S., Murphy, R. A., Sevcik, K. E., Brakke, S. L. \& Williams, S. L. 1993. Language comprehension in ape and child. Monographs of the Society for Research in Child Development, 58, 1-256.

Sayers, K. \& Lovejoy, C. O. 2008. The chimpanzee has no clothes: a critical examination of Pan troglodytes in models of human evolution. Current Anthropology, 49, 87-114.

Schell, A., Rieck, K., Schell, K., Hammerschmidt, K. \& Fischer, J. 2011. Adult but not juvenile Barbary macaques spontaneously recognize group members from pictures. Animal Cognition, 14, 503-509.

Schmitt, V. \& Fischer, J. 2009. Inferential reasoning and modality dependent discrimination learning in olive baboons (Papio hamadryas anubis). Journal of Comparative Psychology, 123, 316-325.

Schmitt, V. \& Fischer, J. 2011. Representational format determines numerical competence in monkeys. Nature Communications, 2, 257. doi: 10.1038/ncomms1262.

Schmitt, V., Pankau, B. \& Fischer, J. 2012. Old World monkeys compare to apes in the PCTB. PLOS ONE, 7, e32024.

Schnoell, A. V. \& Fichtel, C. 2012. Wild refronted lemurs (Eulemur rufifrons) use social information to learn a new foraging technique. Animal Cognition. doi: 10.1007/s10071012-0477-y.

Seed, A., Clayton, N. S., Carruthers, P., Dickinson, A., Glimcher, P. W., Güntürkün, O., Hampton, R. R., Kacelnik, A., Shanahan, M., Stevens, J. R. \& Tebbich, S. 2011. Planning, memory, and decision making. In: Animal Thinking: Contemporary Issues in Comparative Cognition (Ed. by R. Menzel \& J. Fischer), pp. 121-147. Cambridge: MIT Press.

Semendeferi, K., Lu, A., Schenker, N. \& Damasio, H. 2002. Humans and great apes share a large frontal cortex. Nature Neuroscience, 5, 272-276.

Seyfarth, R. M. \& Cheney, D. L. 2007. Primate social knowledge and the origins of language. Mind and Society, 7, 129-142.

Seyfarth, R. M., Cheney, D. L. \& Marler, P. 1980. Monkey responses to three different alarm calls: Evidence of predator classification and semantic communication. Science, 210, 801-803.

Shettleworth, S. J. 2009. The evolution of comparative cognition: Is the snark still a boojum? Behavioural Processes, 80, 210-217.

Shettleworth, S. J. 2010a. Clever animals and killjoy explanations in comparative psychology. Trends in Cognitive Sciences, 14, 477-481.

Shettleworth, S. J. 2010b. Cognition, Evolution and Behavior. New York: Oxford University Press. 
Shettleworth, S. J. 2012. Darwin, Tinbergen, and the evolution of comparative cognition. In: Oxford Handbook of Comparative Evolutionary Psychology (Ed. by J. Vonk \& T. Shackleford). New York: Oxford University Press.

Shifferman, E. 2009. Its own reward: lessons to be drawn from the reversed-reward contingency paradigm. Animal Cognition, 12, 547-558.

Shultz, S. \& Dunbar, R. I. M. 2010. Species differences in executive function correlate with hippocampus volume and neocortex ratio across nonhuman primates. Journal of Comparative Psychology, 124, 252-260.

Silva, F. \& Silva, K. 2006. Humans' folk physics is not enough to explain variations in their toolusing behavior. Psychonomic Bulletin \& Review, 13, 689-693.

Silva, F. J., Silva, K. M., Cover, K. R., Leslie, A. L. \& Rubalcaba, M. A. 2008. Humans' folk physics is sensitive to physical connection and contact between a tool and reward. Behavioural Processes, 77, 327-333.

Simon, R., Holderied, M. W. \& von Helversen, O. 2006. Size discrimination of hollow hemispheres by echolocation in a nectar feeding bat. Journal of Experimental Biology, 209, 3599-3609.

Skinner, B. F. 1938. The Behavior of Organisms: An Experimental Analysis. New York: AppletonCentury.

Spelke, E. S. \& Hespos, S. J. 2001. Continuity, competence, and the object concept. In: Causal Cognition: A Multidisciplinary Debate (Ed. by E. Dupoux), pp. 325-340. Cambridge: MIT Press.

Stoinski, T. S. \& Whiten, A. 2003. Social learnig by orangutans (Pongo abelii and Pongo pygmaeus) in a simulated food-processing task. Journal of Comparative Psychology, 117, 272-282.

Stoinski, T. S., Wrate, J. L., Ure, N. \& Whiten, A. 2001. Imitative learning by captive western lowland gorillas (Gorilla gorilla gorilla) in a simulated food-processing task. Journal of Comparative Psychology, 115, 272-281.

Suarez, S. D. \& Gallup, G. G. 1986. Social responding to mirrors in rhesus macaques (Macaca mulatta): Effects of changing mirror location. American Journal of Primatology, 11, 239244.

Suddendorf, T. \& Whiten, A. 2001. Mental evolution and development: evidence for secondary representation in children, great apes, and other animals. Psychological Bulletin, 127, 629-650.

Suganuma, E., Pessoa, V., Mongefuentes, B., Castro, B. \& Tavares, M. 2007. Perception of the Müller-Lyer illusion in capuchin monkeys (Cebus apella). Behavioural Brain Research, $182,67-72$.

Terrace, H., Petitto, L., Sanders, R. \& Bever, T. 1979. Can an ape create a sentence? Science, 206, 891-902.

Teufel, C., Gutmann, A., Pirow, R. \& Fischer, J. 2010. Facial expressions modulate the ontogenetic trajectory of gaze-following among monkeys. Developmental Science, 13, 913-922.

The Chimpanzee Sequencing and Analysis Consortium. 2005. Initial sequence of the chimpanzee genome and comparison with the human genome. Nature, 437, 69-87. 
Thorndike, E. L. 1898. Animal intelligence. An experimental study of the associative processes in animals. Psychological Review, 551 - 553.

Thorndike, E. L. 1911. Animal Intelligence: Experimental Studies. New York: The Macmillan Company.

Tinbergen, N. 1951. The Study of Instinct. Oxford: Clarendon Press.

Tinbergen, N. 1963. On aims and methods of ethology. Zeitschrift für Tierpsychologie, 20, 410433.

Tomasello, M. 2008. Origins of Human Communication. Cambridge: MIT Press.

Tomasello, M. 2009. Why we cooperate. Cambridge: MIT Press.

Tomasello, M. \& Call, J. 1997. Primate Cognition. New York: Oxford University Press.

Tomasello, M., Call, J. \& Hare, B. 2003a. Chimpanzees understand psychological states - the question is which ones and to what extent. Trends in Cognitive Sciences, 7, 153-156.

Tomasello, M., Call, J. \& Hare, B. 2003b. Chimpanzees versus humans: it's not that simple. Trends in Cognitive Sciences, 7, 239-240.

Tomasello, M. \& Herrmann, E. 2010. Ape and human cognition. Current Directions in Psychological Science, 19, 3-8.

Tomasello, M. \& Moll. 2009.The gap is social: Human shared intentionaltiy and culture. In: Mind the Gap: Tracing the Origins of Human Universals (Ed. by P. M. Kappeler \& J. B. Silk), pp. 331-350. Berlin, Heidelberg: Springer Verlag.

Trivers, R. L. 1972. Parental investment and sexual selection. In: Sexual selection and the descent of man (Ed. by B. Campbell), pp. 136-179. Chicago: Aldine.

Truppa, V., Piano Mortari, E., Garofoli, D., Privitera, S. \& Visalberghi, E. 2011. Same/different concept learning by capuchin monkeys in matching-to-sample tasks. PLOS ONE, 6, e23809.

Tudusciuc, O. \& Nieder, A. 2007. Neuronal population coding of continuous and discrete quantity in the primate posterior parietal cortex. Proceedings of the National Academy of Sciences, 104, 14513-14518.

Tudusciuc, O. \& Nieder, A. 2010. Comparison of length judgments and the Müller-Lyer illusion in monkeys and humans. Experimental Brain Research, 207, 221-231.

Udell, M. A. R., Dorey, N. R. \& Wynne, C. D. L. 2010. What did domestication do to dogs? A new account of dogs' sensitivity to human actions. Biological Reviews, 85, 327-345.

van Schaik, C. 1999. The socioecology of fission-fusion sociality in orangutans. Primates, 40, 6986.

Voelkl, B. \& Huber, L. 2000. True imitation in marmosets. Animal Behaviour, 60, 195-202.

Waldmann, M. R., Hagmayer, Y. \& Blaisdell, A. P. 2006. Beyond the information given: causal models in learning and reasoning. Current Directions in Psychological Science, 15, 307311.

Ward, C. \& Smuts, B. B. 2007. Quantity-based judgments in the domestic dog (Canis lupus familiaris). Animal Cognition, 10, 71-80.

Watanabe, A. \& Takeda, K. 1963. The change of discharge frequency by A.C. stimulus in a weak electric fish. Journal of Experimental Biology, 40, 57-66. 
Watson, J. B. 1925. Behaviorism. New York: W.W. Norton and Company.

Weiss, D. J. \& Santos, L. R. 2006. Why primates? The importance of nonhuman primates for understanding human infancy. Infancy, 9, 127-140.

Wellman, H. M., Lane, J. D., LaBounty, J. \& Olson, S. L. 2011. Observant, nonaggressive temperament predicts theory-of-mind development. Developmental Science, 14, 319326.

Wheeler, B. 2010. Decrease in alarm call response among tufted capuchins in competitive feeding contexts: possible evidence for counterdeception. International Journal of Primatology, 31, 665-675.

Wheeler, B. C. 2009. Monkeys crying wolf? Tufted capuchin monkeys use anti-predator calls to usurp resources from conspecifics. Proceedings of the Royal Society of London Series B: Biological Sciences, 276, 3013-3018.

White, D. J., Ho, L., de los Santos, G. \& Godoy, I. 2007. An experimental test of preferences for nest contents in an obligate brood parasite, Molothrus ater. Behavioral Ecology, 18, 922-928.

Whiten, A., Goodall, J., McGrew, W. C., Nishida, T., Reynolds, V., Sugiyama, Y., Tutin, C., Wrangham, R. W. \& Boesch, C. 2001. Charting cultural variation in chimpanzees. Behaviour, 138, 1481-1516.

Whiten, A., Goodall, J., McGrew, W. C., Nishida, T., Reynolds, V., Sugiyama, Y., Tutin, C. E. G., Wrangham, R. W. \& Boesch, C. 1999. Cultures in chimpanzees. Nature, 399, 682-685.

Wiltschko, W. \& Wiltschko, R. 2005. Magnetic orientation and magnetoreception in birds and other animals. Journal of Comparative Physiology A: Neuroethology, Sensory, Neural, and Behavioral Physiology, 191, 675-693.

Wright, A. A. 2006. Memory processing. In: Comparative Cognition: Experimental Explorations of Animal Intelligence (Ed. by E. A. Wasserman \& T. R. Zentall), pp. 164-185. New York: Oxford University Press.

Yerkes, R. M. 1943, 1971. Chimpanzees: A laboratory colony. New York: Johnson Reprint Corporation.

Yerkes, R. M. \& Yerkes, A. W. 1929. The Great Apes: A Study of Anthropoid Life. New Haven: Yale University Press.

Yocom, A. M. 2010. Physical and Social Cognition in the White-handed Gibbon (Hylobates lar). Dissertation, The Ohio State University.

Zalmout, I. S., Sanders, W. J., MacLatchy, L. M., Gunnell, G. F., Al-Mufarreh, Y. A., Ali, M. A., Nasser, A.-A. H., Al-Masari, A. M., Al-Sobhi, S. A., Nadhra, A. O., Matari, A. H., Wilson, J. A. \& Gingerich, P. D. 2010. New Oligocene primate from Saudi Arabia and the divergence of apes and Old World monkeys. Nature, 466, 360-364.

Zinner, D., Alberts, S. C., Nunn, C. L. \& Altmann, J. 2002. Significance of primate sexual swellings. Nature, 420, 142-143.

Zinner, D., Nunn, C. L., van Schaik, C. P. \& Kappeler, P. M. 2004. Sexual selection and exaggerated sexual swellings of female primates. In: Sexual Selection in Primates: New and Comparative Perspectives (Ed. by P. M. Kappeler \& C. P. van Schaik), pp. 71-89. Cambridge: Cambridge University Press.

Zuberbühler, K. \& Byrne, R. W. 2006. Social cognition. Current Biology, 16, R786-R790. 
References

Zuberbühler, K., Gygax, L., Harley, N. \& Kummer, H. 1996. Stimulus enhancement and spread of a spontaneous tool use in a colony of long-tailed macaques. Primates, 37, 1-12. 


\section{Acknowledgments}

First, I would like to thank Julia Fischer for supervising my thesis, giving me the opportunity to work with the monkeys, and supporting me with much practical advice and scientific competence throughout the last several years. I truly appreciated her trust and thank her for being a great role model. I also thank Hannes Rakoczy, Michael Waldmann and Andreas Nieder for complementing my thesis committee, providing helpful advice and continuous support.

The research presented in this thesis was funded by the Leibniz Graduate School for Primate Neurobiology (NEUROPRIM), the Göttingen Graduate School for Neurosciences, Biophysics and Molecular Biosciences (GGNB), and the Courant Research Centre for the Evolution of Social Behaviour. I am very grateful to management and the animal caretakers of the German Primate Center for their support during my work. Special thanks got to Sarah, Henry, Henning, Yvonne and Marcel. I would also like to thank Uwe Schönmann and Annette Husung for their support.

Special thanks go to Birte Pankau for training the long-tailed macaques with me, being a great help in collecting data, and being a good friend. Furthermore, I would like to thank Christian Schloegl for fruitful discussions, new insights into animal cognition, and helpful advice on experimental designs.

I would also like to thank Iris Kröger and Jenna Kulp for showing me how to work with the baboons. Many people helped collect the data and supported me while working with the monkeys. Special thanks go to Carolina Lara, Sara Jalali, Laura Almeling, Jana Dekrem, AnnaLena Otte, Teresa Kreusch and Nora Lindstrom.

I would like to thank my friends and colleagues from the DPZ, especially the members of the Cognitive Ethology Lab, for providing such a nice and enjoyable working atmosphere, for the many interesting discussions, coffee breaks and evenings full of laughter. Special thanks go to Matthis, Rebecca, Laura, Chris and Brandon for providing valuable comments and English corrections on earlier versions of this manuscript. I also thank Tanja, Gisela, Annie, Philip and Matze for being such good office companions in the past few weeks.

Many thanks go to my family, especially my mother, for their continuous interest and great support. Danke!

Finally: Thank you, Matze, for your encouragement and support throughout the last years and for enriching my life outside of science! 


\section{Curriculum Vitae}

\section{Personal Information}

\begin{tabular}{|c|c|}
\hline Name & Vanessa Anne Schmitt \\
\hline Date of birth & August 13, 1982 \\
\hline Place of birth & Trier \\
\hline Citizenship & German \\
\hline \multicolumn{2}{|l|}{ Education } \\
\hline since 2009 & $\begin{array}{l}\text { Member of the Göttingen Graduate School for Neurosciences, } \\
\text { Biophysics, and Molecular Biosciences (GGNB) - Systems } \\
\text { Neuroscience } \\
\text { Member of the Leibniz Graduate School for Primate } \\
\text { Neurobiology (Neuroprim) }\end{array}$ \\
\hline since 2008 & $\begin{array}{l}\text { Member of the Courant Research Centre Evolution of Social } \\
\text { Behaviour }\end{array}$ \\
\hline since 2008 & $\begin{array}{l}\text { Ph.D. project, Cognitive Ethology Lab, German Primate Center } \\
\text { "Social and Physical Cognition in Old World Monkeys: A } \\
\text { Comparative Perspective" } \\
\text { Supervisor: Prof. Dr. Julia Fischer } \\
\text { Thesis Committee: Prof. Dr. Michael Waldmann } \\
\text { Prof. Dr. Hannes Rakoczy } \\
\text { Prof. Dr. Andreas Nieder }\end{array}$ \\
\hline 2007 & $\begin{array}{l}\text { M.Sc. (Dipl. Biol.), University of Göttingen, Germany } \\
\text { "Understanding of auditory and visual signals in olive baboons } \\
\text { (Papio anubis)" (supervised by Prof. Dr. Julia Fischer) }\end{array}$ \\
\hline 2004-2007 & Studies in Biology, University of Göttingen, Germany \\
\hline 2002-2004 & Studies in Biology, University of Kaiserslautern \\
\hline 2002 & A-Levels (Abitur), Friedrich-Spee-Gymnasium Trier \\
\hline \multicolumn{2}{|c|}{ Work experience } \\
\hline $2007-2008$ & $\begin{array}{l}\text { Research Assistant, Cognitive Ethology Lab, German Primate } \\
\text { Center, Göttingen, Germany }\end{array}$ \\
\hline 2006 & $\begin{array}{l}\text { Research Assistant, Institute for Zoology and Anthropology, } \\
\text { University of Göttingen, Germany }\end{array}$ \\
\hline
\end{tabular}

\section{Publications}

Schmitt, V., Pankau, B. \& Fischer, J. (2012): Old World Monkeys Compare to Apes in the Primate Cognition Test Battery. PLoSOne 7, e32024

Schmitt, V. \& Fischer, J. (2011): Representational format determines numerical competence in monkeys. Nature Communications: 10.1038/ncomms1262

Schmitt, V. \& Fischer, J. (2011): Quantity discrimination tests with macaques. Nature Protocol Exchange: 10.1038/protex.2011 
Schmitt, V. \& Fischer, J. (2009): Inferential reasoning and modality dependent discrimination learning in olive baboons (Papio hamadryas anubis). Journal of Comparative Psychology 12, 316-325

Schmitt, V., Melchisedech, S., Hammerschmidt, K. \& Fischer, J. (2008): Hand preferences in Barbary macaques (Macaca sylvanus). Laterality 13, 143-157 